\title{
Review of the New World Genera of the Leafhopper Tribe Erythroneurini (Hemiptera: Cicadellidae: Typhlocybinae)
}

Christopher H. Dietrich and Dmitry A. Dmitriev 
Illinois Natural History Survey, David L. Thomas, Chief

A Division of the Illinois Department of Natural Resources

\author{
Illinois Natural History Survey \\ Distribution Office \\ I-Building \\ 1816 South Oak Street \\ Champaign, IL 61820
}

Citation:

Dietrich, C.H., and D.A. Dmitriev. 2006. Review of the New World genera of the leafhopper tribe Erythroneurini (Hemiptera: Cicadellidae: Typhlocycbinae). Illinois Natural History Survey Bulletin 37(5):119-190.

\title{
Editor: Charles Warwick
}

\section{US ISSN 0073-4918}

Printed by authority of the State of Illinois

P0102370 - .75M-07-06

Printed with soy ink on recycled and recyclable paper.

Equal opportunity to participate in programs of the Illinois Department of Natural Resources (IDNR) and those funded by the U.S. Fish and Wildlife Service and other agencies is available to all individuals regardless of race, sex, national origin, disability, age, religion, or other non-merit factors. If you believe you have been discriminated against, contact the funding source's civil rights office and/or the Equal Employment Opportunity Officer, IDNR, One Natural Resources Way, Springfield, IL 62702-1271; 217/785-0067; TTY 217/782-9175. 


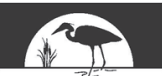

ILLINOIS

NATURAL

HIS TORY

S UR V E Y

\section{Review of the New World Genera of the Leafhopper Tribe Erythroneurini (Hemiptera: Cicadellidae: Typhlocybinae)}

Christopher H. Dietrich and Dmitry A. Dmitriev 


\section{ACKNOWLEDGMENTS}

For lending specimens, we are grateful to Norman Penny (California Academy of Sciences), K.G.A. Hamilton (Canadian National Collection, Ottawa), Boris Kondratieff (Colorado State University), Richard Brown (Mississippi State University), Stuart McKamey and Warren Steiner (National Museum of Natural History, Washington), Luciana Musetti and Creighton Freeman (Ohio State University), and Zachary Falin (University of Kansas). We are also grateful to Gustavo Moya-Raygoza, Harry Brailovsky, and Ricardo Mariño Perez for facilitating field work in Mexico, and to Gerardo Lamas, Juan Grados, Caros Peña, and Pedro Lozada for facilitating field work in Peru. P.H. Freytag, L.L. Deitz, and M.D. Webb provided constructive criticism that greatly improved the manuscript. This work was supported in part by NSF-REVSYS grant DEB0315373 and Hatch award ILLU-875-361 to CHD. 


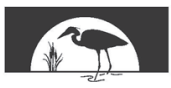

ILLINOIS

NATURAL

HIS TORY

S UR V E Y

\section{Review of the New World Genera of the Leafhopper Tribe Erythroneurini (Hemiptera: Cicadellidae: Typhlocybinae)}

Christopher H. Dietrich and Dmitry A. Dmitriev

Correspondence to:

C.H. Dietrich

Center for Biodiversity

Illinois Natural History Survey

1816 South Oak Street

Champaign IL 61820 USA

217/ 244-7408

dietrich@inhs.uiuc.edu 


\section{CONTENTS}

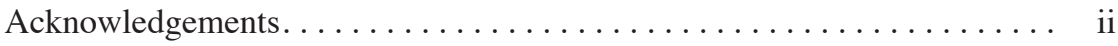

Abstract..................................... 119

Introduction. . . . . . . . . . . . . . . . . . . . . . . 120

Materials and Methods . . . . . . . . . . . . . . . . . . . . . . 121

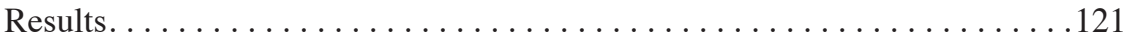

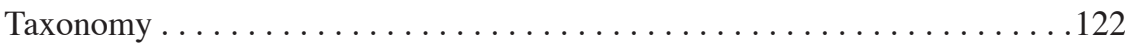

Key to Adult Males of the New World Erythroneurini. . . . . . . . . 122

Status of Previously Described Taxa . . . . . . . . . . . . . 125

Old World Genera that Previously Included New World Species . . .125

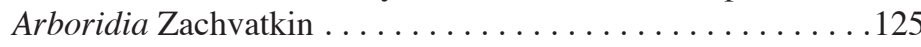

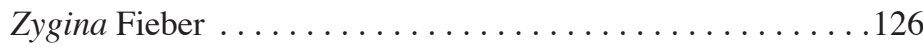

New World Genera . . . . . . . . . . . . . . . . . . . . . . . . . . 126

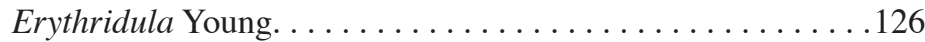

Erythroneura Fitch . . . . . . . . . . . . . . . . . . . 132

Eratoneura Young. . . . . . . . . . . . . . . . . . . 133

Erasmoneura Young . . . . . . . . . . . . . . . . . . 139

Hymetta McAtee. . . . . . . . . . . . . . . . . . . . 140

Descriptions of New Taxa. . . . . . . . . . . . . . . . . . . 140

North American Genera . . . . . . . . . . . . . . . . . . . . . . . . . . . . 140

Aztegina, new genus ....................... 140

Hepzygina, new genus . . .....................

Illinigina, new genus. . . . . . . . . . . . . . . . . . . .142

Mexigina, new genus .......................143

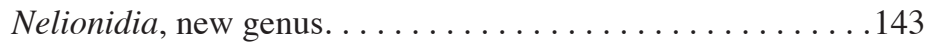

Neoimbecilla, new genus ........................

Neozygina, new genus. . . . . . . . . . . . . . . . 147

Rossmoneura, new genus ......................148

Zyginama, new genus . . . . . . . . . . . . . . . . . . . . 149

South American Genera . . . . . . . . . . . . . . . . . . . . . . . . . . 150

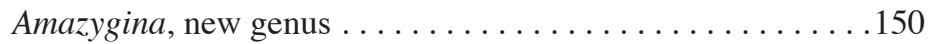

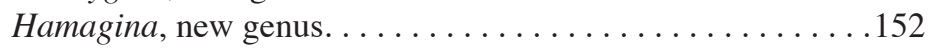

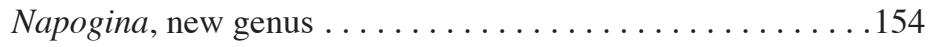

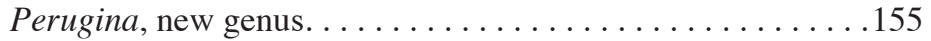

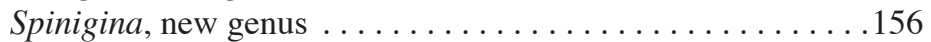

Concluding Remarks. . . . . . . . . . . . . . . . . . . . . . . . . . . .157

Literature Cited . . . . . . . . . . . . . . . . . . . . . . . . . . . . 159

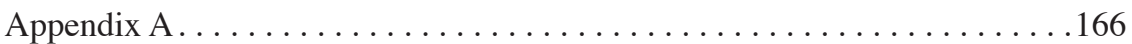

Appendix B . . . . . . . . . . . . . . . . . . . . . . . . . . . . 169

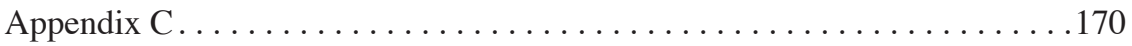

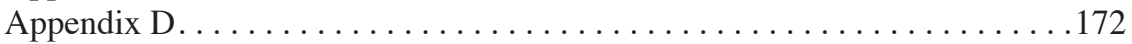

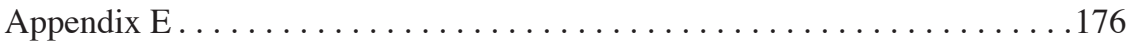

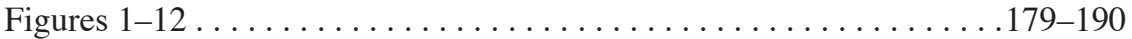




\section{ABSTRACT}

The genus-level classification of New World Erythroneurini is revised based on results of a phylogenetic analysis of 100 morphological characters. The 704 known species are placed into 18 genera. Erasmoneura Young and Eratoneura Young, previously treated as subgenera of Erythroneura Fitch, and Erythridula Young, most recently treated as a subgenus of Arboridia Zachvatkin, are elevated to generic status. Three species previously included in Erasmoneura are placed in a new genus, Rossmoneura (type species, Erythroneura tecta McAtee). The concept of Erythroneura is thereby narrowed to include only those species previously included in the nominotypical subgenus. New World species previously included in Zygina Fieber are not closely related to the European type species of that genus and are therefore placed in new genera. Neozygina, n. gen., based on type species Erythroneura ceonothana Beamer, includes all species previously included in the "ceonothana group", and Zyginama, n. gen., based on type species Erythroneura ritana Beamer, includes most species previously included in the "ritana group" of New World Zygina. Five additional new genera are described to include other previously described North American Erythroneurini: Hepzygina, n. gen., based on type species Erythroneura milleri Beamer and also including E. aprica McAtee; Mexigina, n. gen., based on type species Erythroneura oculata McAtee; Nelionidia, n. gen., based on type species N. pueblensis, n. sp., three additional new species, and Erythroneura amicis Ross; Neoimbecilla, n. gen., based on type species Erythroneura kiperi Beamer and one new species; and Illinigina, n. gen., based on type species Erythroneura illinoiensis Gillette. Five new genera, based on previously undescribed species, are also recognized: Aztegina, n. gen, based on A. punctinota, n. sp., from Mexico; Amazygina, n. gen., based on type species A. decaspina, n. sp., and three additional new species from Ecuador; Hamagina, n. gen., based on type species $H$. spinigera, n. sp., and two additional new species from Peru and Ecuador; Napogina, n. gen., based on type species N. recta, n. sp., and one additional new species from Ecuador; Perugina, n. gen., based on type species P. denticula, n. sp., from Peru; and Spinigina, n. gen., based on type species $S$. hirsuta, n. sp., and an additional new species from Peru. Phylogenetic analysis suggests that the New World Erythroneurini consist of three lineages resulting from separate invasions from the Old World.

\section{KEYWORDS}

Homoptera, identification, leafhopper, morphology, phylogeny, taxonomy 


\section{INTRODUCTION}

The leafhopper subfamily Typhlocybinae is a diverse group of mostly tiny, delicate leafhoppers that feed preferentially on the contents of leaf parenchyma cells of their host plants. Species of this subfamily differ from other leafhoppers in lacking closed preapical cells in the forewing and in having the first hind tarsomere acuminate apically. In agroecosystems, feeding by large populations of typhlocybines causes the familiar "stippling" injury to numerous crops including soybean, potato, apple, and grape. Some species cause a more severe kind of injury known as "hopperburn" (Backus et al. 2005). Currently, Typhlocybinae comprises ca. 5,000 described species worldwide, making it the second largest leafhopper subfamily, after Deltocephalinae. Nevertheless, the diverse tropical faunas of Typhlocybinae, particularly in the New World, remain poorly documented, and it is likely that the group will eventually be found to eclipse all other leafhopper subfamilies in species richness.

The largest tribe of Typhlocybinae appears to be Erythroneurini, with 164 genera and nearly 2,000 described species. Diverse regional faunas of this tribe have been documented in the Old World tropics, particularly on the Indian subcontinent and in southeast Asia (Oman et al. 1990). Tropical parts of Africa, Madagascar, Australia, and New Guinea also appear to harbor diverse erythroneurine faunas, but these remain largely undescribed.

The previously documented New World fauna of erythroneurine leafhoppers comprises over 700 described species, all from North America. Available host plant records indicate that the vast majority of North American species feed and oviposit on woody angiosperms. Most species appear to specialize on a particular host genus or species for oviposition and nymphal development, although adults often utilize other food plants during spring and fall (Ross and DeLong 1953). Species are particularly diverse and abundant in eastern deciduous forests, from which the majority of species have been described, and where as many as 100 species have been collected from a single tree (Hepner 1976). Detailed information on the life history and ecology of the Erythroneurini of eastern North America was provided by Ross and DeLong (1953). Little is known about the ecology of the many species that occur in the southwestern U.S., Mexico, and the Neotropics.

Prior to 1952, with the exception of seven species of Hymetta McAtee, all New World species of Erythroneurini were placed in the genus Erythroneura Fitch. In his revision of the North American fauna, Beamer (1930, 1931a, b, c, d, 1932a, c, d, e, f, g, h, 1938, 1946) recognized four species groups within Erythroneura based primarily on the venation and markings of the forewing: the comes group, the maculata group, the vulnerata group, and the obliqua group. Young (1952) identified additional features in the male genitalia supporting these groups and formally recognized them as subgenera by naming them, respectively, Erythroneura (sensu stricto), Eratoneura Young, Erasmoneura Young, and Erythridula Young. Young (1952) excluded some predominantly southwestern U.S. species from Erythroneura because the male style of these species lacks a second apical extension. These he assigned to Zygina Fieber, while noting that the genus as then defined constituted a "complex of considerable size and wide distribution ...doubtless destined to undergo considerable subdivision."

Beginning in the 1970s, Dworakowska (1970a and numerous subsequent papers) embarked on a long-term project to revise the Old World fauna of Erythroneurini, reassigning nearly all Old World species previously placed in Erythroneura and Zygina to other genera and describing over 500 new species, largely from the Afrotropical and Oriental regions. To date, Dworakowska and others have described more than 150 genera and subgenera to comprise the approximately 1,200 described Old World species of Erythroneurini.

Progress on the classification of New World Erythroneurini has lagged behind that of the Old World fauna. While nearly 300 North American species have been described in the past 50 years, mostly by Hepner (1966 and subsequent papers), endemic New World species of erythroneurine leafhoppers have continued to be placed into only four genera: Arboridia Zachvatkin (subgenus Erythridula), Erythroneura, Hymetta McAtee, and Zygina Fieber (Young 1952, Dworakowska 1970b). Moreover, although Young (1952: 75) examined specimens of an undescribed species of the "ceonothana group" of Zygina from Argentina, not a single species of Erythroneurini, the most speciose group of typhlocybine leafhoppers in the Old World tropics, has been described from 
South America. This paper is a first attempt to elucidate the phylogenetic status and relationships of New World Erythroneurini and to align the generic concepts of this fauna more closely with those currently applied to the Old World fauna.

\section{MATERIALS AND METHODS}

To examine the phylogenetic relationships within Erythroneurini and determine the status of the New World genera, a matrix of 100 adult morphological characters was compiled for 75 exemplar Erythroneurini species and 10 outgroups. The taxon sample comprised two or more representatives of each of the previously recognized and newly described New World genera and subgenera, including the type species when possible. It was not practical to include representatives of all the recognized genera of Erythroneurini in the analysis, so exemplars of Old World genera were selected to represent a broad spectrum of the morphological diversity of the tribe. Exemplars of the typhlocybine tribes Alebrini, Dikraneurini, Empoascini, Jorumini, and Typhlocybini were included as outgroups. Phylogenetic analyses of this matrix were performed using PAUP* 4.0 (Swofford 1998) with TBR branch swapping on 100 random taxon addition sequence replicates. Multistate characters were treated as non-additive (unordered) and trees were rooted to the outgroup taxon Paralebra sp. (Alebrini). Branch support was measured by calculating the decay index (Bremer 1994) for each consistently resolved node on the most parsimonious trees. This was accomplished using the "enforce topological constraints" option to search for the shortest tree(s) not compatible with each node, using the heuristic search algorithm in PAUP*. Characters and states are listed in Appendix A. A list of species examined is given in Appendix B. The data matrix is given in Appendix C.

Morphological terminology follows Young (1952) with the following exceptions. Wing venation follows Shcherbakov $(1981,1982)$, with some abbreviation to reflect the reduced venation of typhlocybine leafhoppers; veins and cells are named as shown in Figs. 1-B and C. Leg chaetotaxy follows Rakitov (1998), with rows of setae designated according to their position when the leg is extended laterad (i.e., $\mathrm{AV}=$ anteroventral; $\mathrm{AM}=$ anteromedial $; \mathrm{PD}=$ posterodorsal, etc.). For simplicity, the angulate projections, or points, of the male style (paramere) apex are numbered 1-3 as shown in Fig. 1-H. As in most leafhopper taxa, identification of erythroneurine species, and in many cases genera, requires clearing and examination of the male genital capsule and genitalia. Thus, keys and descriptions are based on males. At present, females of most species may only be identified through their association with males.

Specimens of newly described taxa are deposited in the insect collections of the Illinois Natural History Survey, Champaign (INHS), the U.S. National Museum of Natural History, Washington (USNM), and the Universidad Nacional Mayor de San Marcos, Lima, Peru (USML). Other specimens, including primary types of previously described species, were examined from the California Academy of Sciences, San Francisco; Canadian National Collection, Ottawa; Colorado State University, Ft. Collins; Mississippi State University, State College; North Carolina State University, Raleigh; Ohio State University, Columbus; University of Kansas, Lawrence; INHS; and USNM.

Interpretations and synonymies of previously described species follow Young (1952) and the more recent literature, unless otherwise noted. In the lists of species included in each genus, only species presently considered valid, and new synonyms, are listed. More complete synonymies will be included in subsequent treatments of the individual genera (in preparation).

\section{RESULTS}

The phylogenetic analysis of morphological data recovered 24 equally parsimonious trees of length 1056, rescaled consistency index 0.100 , and retention index 0.571 . The strict consensus ( Fig. 12) of these trees is well resolved with only three unresolved trichotomies. The analysis recovered Erythroneurini as monophyletic with sister group Dikraneurini. Most erythroneurine taxa were grouped into two large clades subtended by a paraphyletic grade of tropical Australasian taxa. The newly described South American genera Spinigina, Napogina, Hamagina, Perugina, and Amazygina together formed a monophyletic group that was sister to a clade comprising primarily Afrotropical genera. The North American genera (including Neozygina and 
Zyginama, which are also represented in South America) grouped together in a large clade that also included some East Asian taxa (Ziczacella, Arboridia spp., and Asianidia).

The analysis supported the monophyly of the North American genera Hymetta, Erythroneura (sensu stricto), Erasmoneura, Hepzygina, n. gen., Neozygina, n. gen., Zyginama, n. gen., and Nelionidia, n. gen. Eratoneura was paraphyletic with respect to Erythroneura, and constraining Eratoneura to be monophyletic required five additional steps. Erythridula and Neoimbecilla were also paraphyetic on the most parsimonious trees. Yet, constraining these genera as monophyletic added only a single step to the length of the tree. The analysis also suggested that Arboridia (sensu lato) as currently defined is polyphyletic. The New World species previously placed in Zygina did not group with the included Old World representative of the genus. Most of the former grouped together into a clade comprising the newly described genera Neozygina (corresponding to the informal "ceonothana group" recognized by Young), Zyginama (comprising most species previously included in the "ritana species group"), Mexigina and Hepzygina (comprising additional "ritana group" species). Two additional species of the "ritana group," were placed in a clade with Hymetta, and these are here included in two new genera-Illinigina and Neoimbecilla.

The analysis largely supports Ross' (1965) hypothesis that the New World fauna of Erythroneurini originated through multiple invasions from the Old World. On the resulting cladograms, alternative equally parsimonious optimizations of the binary character "geographic distribution," with states (0) Old World and (1) New World, are possible. Under the parsimony criterion, the results require at least three, and are consistent with as many as four, independent origins of the various New World erythroneurine lineages (Fig. 12). A clade comprising the endemic South American genera was sister to a clade comprising mostly Afrotropical genera. The North American genera grouped into two clades, each of which had an Asian Palaearctic taxon as its sister. Nevertheless, because many of the nodes involved received low branch support, these scenarios remain questionable. Constraining the New World Erythroneurini to be monophyletic requires a minimum of nine additional steps and constraining the North American Erythroneurini to be monophyletic requires at least seven additional steps.

\section{TAXONOMY}

\section{Tribe Erythroneurini Young, 1952}

This tribe is a well-supported monophyletic group, characterized by the venation of the hindwing, in which the anal vein is completely confluent with the postcubital vein (Fig. 1-C: $\mathrm{v}$ ), the submarginal vein is absent between RM and $\mathrm{CuA}$, and the forewing inner (fourth) apical cell is elongate and parallel-sided. Other morphological features that appear to distinguish most members of Erythroneurini from other Typhlocybinae include the relatively broad maxillary plate, which is visible laterad of lorum throughout its length (Fig. 1-A); and the absence of an enlarged AM1 seta near the apex of the front femur (Fig. 1-D).

\section{Key to Adult Males of the New World Genera of Erythroneurini}

1 Pygofer with dorsal appendage absent (small teeth may be present) (Figs. 4-I, 5-L)....................2 1 ' Pygofer with dorsal appendage well developed (may be small), rigidly attached (Fig. 1-I) or articulated to dorsal margin (Fig. 4-A)

2 Pygofer with well developed ventral appendage, dorsal margin without teeth (Fig. 4-I); head with pair of black spots just dorsad of antennal pits in addition to paired spots on crown (southwestern US and Mexico) Mexigina, n. gen.

2' Pygofer without ventral appendage, dorsal margin with small teeth distally (Fig. 5-L); head without pair of black spots just dorsad of antennal pits (South America) Perugina, n. gen. 
3 Pygofer with ventral appendage well developed (Fig. 1-I) (rarely, appendage absent and posteroventral margin with strongly sclerotized ridge), dorsal appendage immovably fused to dorsal margin; first segment of anal tube without spinose posterolateral lobe

3' Pygofer without ventral appendage (or sclerotized ridge), or if small appendage present, first segment of anal tube with spinose posterolateral lobe (Fig. 5-M); dorsal pygofer appendage articulated or fused

4 Anteclypeus (Fig. 1-A) not swollen, extended to lateral margin of face only near apex; subgenital plate with angulate basolateral projection (Fig. 6-A); connective without median anterior lobe (Fig. 1-I)

4' Anteclypeus strongly swollen and very broad, extended to lateral margin of face a considerable distance from apex; subgenital plate without angulate basolateral projection; connective with distinct median anterior lobe ......................................................Alnetoidia alneti (Dahlbom) (Palaearctic species, established in Nova Scotia (Hamilton 1985))

5 Pygofer with 1-2 macrosetae just basad of dorsal appendage, ventrolateral setae inconspicuous or absent (Fig. 5-B); crown weakly produced, anterior and posterior margins parallel, with pair of well delimited brown or black preapical spots (Fig. 2-M).......................Neozygina, n. gen. 5' Pygofer without macrosetae just basad of dorsal appendage, ventrolateral setae usually large, conspicuous (Fig. 1-E); crown with anterior margin usually more produced than posterior margin, with or without paired prepical spots (Fig. 2-O)

6 Aedeagus with large unpaired dorsal process between phallobase and gonopore-bearing shaft (Fig. 9-A); subgenital plate with or without large dorsoapical spine (Fig. 5-H)....Hamagina, n. gen. 6' Aedeagus without large unpaired dorsal process between phallobase and gonopore-bearing shaft; subgenital plate without dorsoapical spine (Fig. 1-I) .................................... ginama, n. gen.

7 Style apex attenuate or broad and truncate, often footlike, apex with no more than two points

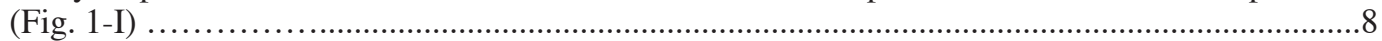
7' Style apex with three points (third point sometimes reduced to obtusangular projection) (Fig. 1H)

8 Pygofer lobe and dorsal membrane conspicuously clothed with long fine setae; connective with well-developed median anterior lobe; subgenital plate without basolateral row of three or more macrosetae ........................................................ Zygina flammigera (Fourcroy) (Palaearctic species, established in coastal western North America (Hamilton 1983)) 8' Pygofer lobe and dorsal membrane without conspicuous long fine setae; connective (Fig. 1-I) without median anterior lobe; subgenital plate with basolateral row of three or more macrosetae

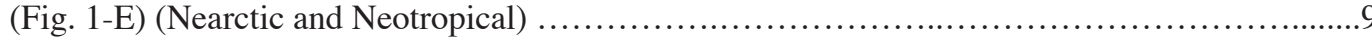

9 Pygofer with dorsal appendage fused to margin, without basal articulation or line of weakening (Fig. 1-E); dorsal apodeme of aedeagus without sclerotized connection to pygofer.......................10 9' Pygofer with dorsal appendage either freely articulated or with distinct basal line of weakening separating it from pygofer margin (Fig. 4-A); dorsal apodeme of aedeagus connected to pygofer by pair of sclerotized arms (Figs. 6-I, J)

10 Aedeagus with paired processes, when present, arising near base (Fig. 8E, F); with or without large unpaired process arising either anterodorsad or posteroventrad of gonopore-bearing shaft ...

10' Aedeagus with paired distal processes present (Fig. 1-G), without large unpaired process other than gonopore-bearing shaft 
11 Unpaired aedeagal process, when present, arising anterodorsad of gonopore-bearing shaft (Figs. 8-I, M); scutellum apex pale; crown unmarked or with unpaired medial macula (South America) Amazygina, n. gen. $11^{\prime}$ Unpaired aedeagal process arising posteroventrad of gonopore-bearing shaft (Fig. 6-F); scutellum apex black; crown with or without paired spots (Mexico and USA) Hepzygina, n. gen.

12 Pygofer with basolateral setae fine, inconspicuous (Fig. 4-F); style apex short, footlike, with imbricate texture (Fig. 6-E) (Mexico) ........................................Aztegina, n. gen. 12' Pygofer with basolateral setae enlarged, conspicuous (Fig. 5-J); style apex usually not footlike (Fig. 10-C), but if so, then glabrous (Fig. 10H) (South America) ....

13 First segment of anal tube with pair of spinose posterolateral lobes; pygofer with appendage arcuate (Figs. 5-M, N) ............................................................Spinigina, n. gen. 13' First segment of anal tube without pair of spinose posterolateral lobes; pygofer with appendage not elevated above margin of distal lobe (Figs. 5-J, K) Napogina, n. gen.

14 Forewing (Figs. 2-E, 3-E) broad, marked with numerous small red or brown flecks, scutellum elevated and shelflike posteriorly .................................................................Hymetta McAtee 14' Forewing (Figs. 2-H, 3-H) narrow, with few larger brown or red spots, scutellum not elevated posteriorly .Illinigina, n. gen.

15 Pygofer appendage rigidly attached to pygofer, without basal suture (Fig. 4-C); crown without pair of brown spots preapically; forewing vein $\mathrm{CuA}$ usually shorter than $\mathrm{CuP}$, base of inner apical cell usually with small dark spot (Fig. 3-B)

15' Pygofer appendage movably articulated to pygofer (Fig. 4-A) or at least with basal line of weakening (or, if pygofer appendage rigidly attached, then crown with pair of brown spots preapicaly); forewing vein CuA approximately same length as CuP, base of inner apical cell usually without small dark spot (Figs. 3-D, G)

16 Forewing with base of inner apical cell transverse (Fig. 1-B), CuA very short or absent, second apical cell often with brown spot near apex; pygofer appendage $\mathrm{C}$-shaped (ventral arm of $\mathrm{C}$ may be branched), without digitiform basal process (Fig. 4-B); aedeagal shaft usually without denticuli ..Erythroneura Fitch 16' Forewing with base of inner apical cell oblique, CuA usually longer, second apical cell without dark spot apically (Fig. 3-C); pygofer appendage a simple spinelike process (Fig. 4-C), bifid in some species, with small digitiform internal process at base; aedeagal shaft usually with denticuli

Eratoneura Young

17 Style apex with third point greatly elongate and curved mesad, much longer than distance between first two points (Figs. 7-Q, T) ... .Neoimbecilla, $\mathrm{n}$. gen. $17^{\prime}$ Style apex with third point short and straight, usually little if any longer than distance between first two points (Fig. 1-H) .

18 Pygofer with basolateral setae undifferentiated, inconspicuous; dorsal coloration usually predominantly pale yellow or white with bold longitudinal orange or red lines; crown without pair of brown preapical spots (Fig. 2-A) .... Erythridula Young 18' Pygofer basolateral setae well differentiated, conspicuous (Fig. 4-D); dorsal coloration various, usually dark orange or brown with paler markings, never consisting of bold longitudinal lines; crown with or without pair of brown preapical spots (Figs. 2-D, J)

19 Aedeagal shaft elongate, slender, with pair of long recurved lateral processes apically and median anterior preapical lobe or spine, preatrium much shorter than shaft (Fig. 7-A); crown pale with pair of dark preapical spots (Fig. 2-J) Nelionidia, n. gen. 19' Aedeagal shaft short, paired processes short or absent, preatrium longer than shaft; crown with or without dark preapical spots 
20 Aedeagal shaft (Figs. 7-Y, Z) without paired spines or processes, with unpaired posterior preapical spine; crown only slightly longer medially than next to eye, usually with pair of dark preapical spots (Fig. 2-N) ...............................................Rossmoneura, n. gen. 20' Aedeagal shaft with paired spines or processes, without unpaired posterior preapical spine; crown distinctly longer medially than next to eye, usually mostly dark brown (Fig. 2-D), but if pale, then without pair of dark preapical spots

Erasmoneura Young

\section{Status of Previously Described Taxa}

\author{
Old World Genera that Previously Included \\ New World Species
}

\section{Arboridia Zachvatkin}

Arboridia Zachvatkin 1946:153 (type species Typhlocyba parvula Boheman)

Khoduma Dworakowska 1972b:153 (type spe cies: K. jacobii Dworakowska)

Notes. Arboridia was originally described by Zachvatkin (1946), as a subgenus of Zyginidia Haupt, to accommodate a number of western Palaearctic species, including those placed by Ribaut (1936) in his "Erythroneura parvula group." Dworakowska (1970b) elevated Arboridia to generic rank and transferred the subgenus Erythridula Young (erected by Young, 1952, as a subgenus of Erythroneura) to Arboridia, continuing to treat the former as a subgenus. She noted that Erythridula differs from Arboridia (sensu stricto) in having the head more strongly produced, the face wider ventrally, the second apical cell of the forewing narrower and the fourth wider. She also noted that Erythridula and Arboridia differ dramatically in coloration. Most Erythridula species have the dorsum pale overall with bold longitudinal red or orange stripes on the head and thorax (including forewing); they also lack paired black spots on the vertex. In contrast, Palaearctic species of Arboridia (s.s.) lack bold longitudinal stripes, the crown has a pair of black preapical spots, the mesonotum has black basolateral triangles, and the body including the abdomen is heavily marked with dark brown or black. Although the structure of the male genital capsule and genitalia of Arboridia (s.s.) and Erythridula are very similar, species of the latter have the macrosetae of the subgenital plate considerably larger and male sternite IX usually has a median longitudinal internal ridge anteriorly that is usually absent in Arboridia (s.s.).
European species of Arboridia also have the male subgenital plates broader overall with the lateral margin rounded basally, and the ventral angle of the style apex is situated distad of the dorsal fold of the subgenital plate. Erythridula have the plates much narrower with the lateral margin distinctly angulate basally, and the ventral angle of the style apex is even with or basad of the dorsal fold of the subgenital plate. These features, in combination with other differences noted by Dworakowska, as well as the non-overlapping geographic distributions of the two groups, seem sufficient to justify elevating Erythridula to generic rank (see below), thus restricting Arboridia to include only the nominotypical subgenus and subgenus Arborifera Sohi and Sandhu (misspelled as "Arboridula" by Dworakowska and Viraktamath 1975 and Dworakowska 1980).

Species from the Oriental region currently placed in Arboridia (Dworakowska and Viraktamath 1975; Dworakowska 1979, 1980; Sohi and Dworakowska 1984), although sharing similar pygofer structure and chaetotaxy with both the Palaearctic Arboridia and the Nearctic Erythridula, differ considerably in other respects. These species mostly lack welldeveloped paired spots on the crown and longitudinal stripes on the dorsum, and many lack a third point at the style apex.

The morphology-based phylogenetic analysis, which included five species of Erythridula and seven representatives of Old World Arboridia, grouped Erythridula in a paraphyletic grade (one step shorter than a tree constrained to include a monophyletic Erythridula) but indicated that the Old World Arboridia may be polyphyletic (Fig. 12). Thus, although some Oriental species of Arboridia have already been placed in a separate subgenus (Arborifera), one or more additional genera or subgenera may eventually need to be erected to receive various "Arboridia" species from the Oriental region and the Middle East. 
Under the concept of the genus adopted here, Arboridia is restricted to the Old World.

\section{Zygina Fieber}

Zygina Fieber 1866a:509 (type species: Typhlo cyba nivea Mulsant and Rey)

Flammigeroidia Dlabola 1958c:56 (type species: "Erythroneura flammigera Geoffr" = Cicada flammigera Fourcroy)

Notes. In his classification of Western Hemisphere Typhlocybinae, Young (1952) redefined Zygina, which previously had been treated as a synonym of Erythroneura by Ribaut (1936), to include "all those leafhoppers with a single broad extension of the style apex." Based on their lack of a third point ("second extension" in Young's terminology) of the male style, Young placed in Zygina all New World erythroneurines that had been included by previous authors in the informal "Western Obliqua group" of Erythroneura (DeLong and Knull 1946).

Although Young's revision treated only the New World fauna, he noted that Oriental species included in Zygina by Matsumura (1931) appeared to have been placed accurately and that many additional Old World erythroneurine species were referable to the "Zygina complex." Some subsequent authors followed Young's broad definition of the genus and placed various New Zealand, African, and Oriental species in Zygina.

More recently, the definition of Zygina has been narrowed considerably and Dworakowska (1970c) included only Palaearctic species. Under this definition, the genus includes species having the following combination of features: male pygofer with conspicuous setae laterally and on dorsal membrane, dorsal appendage small, slender, and not movably articulated; subgenital plate with basolateral projection absent and macrosetae distributed mostly beyond midlength; style apex with two points, preapical lobe weakly developed.

Excluded from this definition are all New World species included in Zygina by Young (1952). Phylogenetic analysis indicated that these species are not closely related to the Palaearctic species of the genus. Based on this analysis, the species are here included in seven new genera, described below.

One Palaearctic species, Zygina flammigera (Fourcroy), has become established in the New
World and is recorded from Washington state and British Columbia (Hamilton 1983).

\section{New World Genera}

\section{Erythridula Young, 1952, new status}

Erythroneura (Erythridula) Young 1952:81

(type species: Tettigonia obliqua Say)

Arboridia (Erythridula) Dworakowska 1970b:615

Diagnosis. This genus differs from other New World Erythroneurini in having the following combination of features: ground color usually pale, often with bold longitudinal red or orange stripes (Fig. 2-A); crown without pair of brown or black spots; forewing apical cells without dark spots, inner apical cell with base oblique (Fig. 3-A); male pygofer without ventral appendage or sclerotized ridge, dorsal appendage freely articulated, basolateral setae fine and inconspicuous (Fig. 4-A); style apex with three angulate projections.

Distribution. Temperate North America.

Notes. The phylogenetic analysis recovered Erythridula as paraphyletic, but constraining the included species to form a monophyletic group added only one step to the tree. Despite the lack of support for a monophyletic Erythridula, species in this genus are readily distinguishable from other North American Erythroneurini by the features given in the key. Thus, Erythridula is treated as a distinct taxon, pending more detailed analyses. As noted above, despite similarities in the structure of the male genital capsule, consistent differences in external morphology suffice to justify treating Erythridula as a distinct genus, rather than as a subgenus of Arboridia. Erythridula presently comprises 256 species, all of which are apparently restricted to North America. A specimen-level database and interactive key to species are available on-line at http://ctap.inhs.uiuc.edu/dmitriev/3i_keys. asp. 


\section{Included species:}

Erythridula abolla (McAtee, 1920a:285) (Erythroneura), new combination Erythridula accurata (Beamer, 1934b:18 n.nov.) (Erythroneura), new combination Erythridula acicularis (Beamer, 1932i:126) (Erythroneura), new combination Erythridula acutalis (Ross \& DeLong, 1953a:83) (Erythroneura), new combination Erythridula adae (Hepner, 1977c:363) (Erythroneura), new combination Erythridula ador (McAtee, 1918b:361) (Erythroneura), new combination Erythridula aenea (Beamer, 1930b:436) (Erythroneura), new combination Erythridula aesculella (Ross \& DeLong, 1953a:82) (Erythroneura), new combination Erythridula afflicta (Beamer, 1935a:101) (Erythroneura), new combination Erythridula alata (Knull, 1946a:45) (Erythroneura), new combination Erythridula albanyensis (Hepner, 1976b:124) (Erythroneura), new combination Erythridula albescens (Beamer, 1930b:443) (Erythroneura), new combination Erythridula amabilis (McAtee, 1924d:132) (Erythroneura), new combination Erythridula ampaiae (Hepner, 1976d:314) (Erythroneura), new combination Erythridula ampasa (Ross \& DeLong, 1953a:86) (Erythroneura), new combination Erythridula ampla (Knull, 1951c:179) (Erythroneura), new combination Erythridula andrewsi (Hepner, 1976b:121) (Erythroneura), new combination Erythridula angularis (Beamer, 1930b:447) (Erythroneura), new combination Erythridula anomala (Knull, 1946a:46) (Erythroneura), new combination Erythridula apta (Beamer, 1935a:102) (Erythroneura), new combination Erythridula aspera (Beamer \& Griffith, 1935a:18) (Erythroneura), new combination Erythridula atrimucronata (Beamer, 1930b:424) (Erythroneura), new combination Erythridula autenae (Johnson, 1935a:73) (Erythroneura), new combination Erythridula barbarae (Hepner, 1978a:134) (Erythroneura), new combination Erythridula barnesae (Hepner, 1976d:313) (Erythroneura), new combination Erythridula beckiae (Hepner, 1978a:131) (Erythroneura), new combination Erythridula belindae (Hepner, 1976a:207) (Erythroneura), new combination Erythridula benedicti (Hepner, 1976b:124) (Erythroneura), new combination Erythridula betulaspera (Richards \& Varty, 1964a:515) (Erythroneura), new combination Erythridula bicornis (Beamer, 1930b:450) (Erythroneura), new combination Erythridula bitincta (McAtee, 1926c:130) (Erythroneura), new combination Erythridula boniorum (Hepner, 1976b:125) (Erythroneura), new combination Erythridula bowmanorum (Hepner, 1977c:362) (Erythroneura), new combination Erythridula brundusa (Robinson, 1924b:155) (Erythroneura), new combination Erythridula brundusoides (Hepner, 1976d:315) (Erythroneura), new combination Erythridula caldwelli (Johnson, 1935a:69) (Erythroneura), new combination Erythridula camirei (Hepner, 1976b:119) (Erythroneura), new combination Erythridula carmiensis (Hepner, 1976d:316) (Erythroneura), new combination Erythridula caryaglabrae (Hepner, 1977a:250) (Erythroneura), new combination Erythridula cauta (Beamer, 1935a:100) (Erythroneura), new combination Erythridula cautoides (Hepner, 1977c:363) (Erythroneura), new combination Erythridula cavena (Auten \& Johnson, 1936a:61) (Erythroneura), new combination Erythridula celebrata (Johnson, 1935a:82) (Erythroneura), new combination Erythridula chambersi (Hepner, 1976a:208) (Erythroneura), new combination Erythridula chandleri (Hepner, 1976c:296) (Erythroneura), new combination Erythridula clarysae (Hepner, 1976c:299) (Erythroneura), new combination Erythridula clavata (DeLong, 1916a:105) (Typhlocyba), new combination Erythridula clavatoides (Hepner, 1978a:131) (Erythroneura), new combination Erythridula cliffordi (Hepner, 1976c:293) (Erythroneura), new combination Erythridula cliftoni (Hepner, 1977c:363) (Erythroneura), new combination Erythridula clydei (Hepner, 1977c:363) (Erythroneura), new combination Erythridula coarctata (Beamer, 1930b:436) (Erythroneura), new combination Erythridula coleyi (Hepner, 1977a:254) (Erythroneura), new combination 
Erythridula collinsi (Hepner, 1977c:364) (Erythroneura), new combination Erythridula complicata (Johnson, 1935a:87) (Erythroneura), new combination Erythridula contrasta (Auten \& Johnson, 1936a:62) (Erythroneura), new combination Erythridula cooni (Hepner, 1976b:121) (Erythroneura), new combination Erythridula cornipes (Beamer, 1930b:449) (Erythroneura), new combination Erythridula cotidiana (Beamer, 1930b:433) (Erythroneura), new combination Erythridula crataegi (Johnson, 1935a:61) (Erythroneura), new combination Erythridula crevecoeuri (Gillette, 1898a:767) (Typhlocyba), new combination Erythridula crossi (Hepner, 1976a:210) (Erythroneura), new combination Erythridula cruciformis (Beamer, 1930b:443) (Erythroneura), new combination Erythridula cuneata (Beamer, 1930b:433) (Erythroneura), new combination Erythridula cuneatoides (Hepner, 1977c:364) (Erythroneura), new combination Erythridula curtaega (Hepner, 1976d:313) (Erythroneura), new combination Erythridula davichi (Hepner, 1977a:248) (Erythroneura), new combination Erythridula decorata (Auten \& Johnson, 1936a:63) (Erythroneura), new combination Erythridula dianae (Hepner, 1976a:205) (Erythroneura), new combination Erythridula diffisa (Beamer, 1930b:434) (Erythroneura), new combination Erythridula divisa (McAtee, 1924c:37) (Erythroneura), new combination Erythridula dolosa (Beamer \& Griffith, 1935a:19) (Erythroneura), new combination Erythridula dorisae (Hepner, 1976a:204) (Erythroneura), new combination Erythridula dowelli (Beamer, 1932b:62) (Erythroneura), new combination Erythridula dunni (Hepner, 1976c:295) (Erythroneura), new combination Erythridula edgari (Hepner, 1976c:300) (Erythroneura), new combination Erythridula electa (McAtee, 1920a:282) (Erythroneura), new combination Erythridula eluta (McAtee, 1920a:277) (Erythroneura), new combination Erythridula enata (Knull, 1951c:179) (Erythroneura), new combination Erythridula enatoides (Hepner, 1977c:365) (Erythroneura), new combination Erythridula enfieldensis (Hepner, 1976b:126) (Erythroneura), new combination Erythridula extima (Beamer, 1939a:29) (Erythroneura), new combination Erythridula extimoides (Hepner, 1976d:314) (Erythroneura), new combination Erythridula fagiphylla (Hepner, 1977a:254) (Erythroneura), new combination Erythridula falcata (Beamer, 1930b:432) (Erythroneura), new combination Erythridula florida (Ross \& DeLong, 1953a:82) (Erythroneura), new combination Erythridula floridoides (Hepner, 1978a:133) (Erythroneura), new combination Erythridula fragilis (Johnson, 1935a:93) (Erythroneura), new combination Erythridula frazieri (Hepner, 1976a:210) (Erythroneura), new combination Erythridula freta (Knull, 1951c:179) (Erythroneura), new combination Erythridula fretoides (Hepner, 1977c:364) (Erythroneura), new combination Erythridula frisoni (Ross \& DeLong, 1953a:80) (Erythroneura), new combination Erythridula fultonae (Hepner, 1976d:314) (Erythroneura), new combination Erythridula fulvocephala (Robinson, 1924b:155) (Erythroneura), new combination Erythridula fumida (Gillette, 1898a:758) (Typhlocyba), new combination Erythridula funesta (Beamer, 1930b:441) (Erythroneura), new combination Erythridula furcillata (Beamer, 1930b:452) (Erythroneura), new combination Erythridula garretsoni (Hepner, 1976c:295) (Erythroneura), new combination Erythridula gladysae (Hepner, 1976d:315) (Erythroneura), new combination Erythridula gleditsia (Beamer, 1930b:437) (Erythroneura), new combination Erythridula hamata (Beamer, 1930b:446) (Erythroneura), new combination Erythridula hamiltoni (Hepner, 1977b:52) (Erythroneura), new combination Erythridula hamlinorum (Hepner, 1977c:362) (Erythroneura), new combination Erythridula harei (Hepner, 1977a:255) (Erythroneura), new combination Erythridula harpax (Beamer, 1930b:432) (Erythroneura), new combination Erythridula harrisi (Hepner, 1976a:210) (Erythroneura), new combination Erythridula haspata (Ross \& DeLong, 1953a:85) (Erythroneura), new combination 
Erythridula herberti (Hepner, 1976d:312) (Erythroneura), new combination Erythridula hildae (Hepner, 1977c:362) (Erythroneura), new combination Erythridula hormchunae (Hepner, 1976b:120) (Erythroneura), new combination Erythridula idonea (Beamer, 1935a:100) (Erythroneura), new combination Erythridula ilexae (Hepner, 1976d:315) (Erythroneura), new combination Erythridula ilicis (Ross, 1953b:189) (Erythroneura), new combination Erythridula inconspicua (Johnson, 1935a:90) (Erythroneura), new combination Erythridula infinita (Beamer, 1930b:446) (Erythroneura), new combination Erythridula insigna (Beamer \& Griffith, 1935a:17) (Erythroneura), new combination Erythridula intricata (Johnson, 1935a:86) (Erythroneura), new combination Erythridula isei (Hepner, 1977b:54) (Erythroneura), new combination Erythridula ivae (Hepner, 1976c:297) (Erythroneura), new combination Erythridula ivani (Hepner, 1976d:312) (Erythroneura), new combination Erythridula jeanae (Hepner, 1976a:205) (Erythroneura), new combination Erythridula joanneae (Hepner, 1978a:133) (Erythroneura), new combination Erythridula jocosa (Beamer, 1935a:101) (Erythroneura), new combination Erythridula jonesi (Hepner, 1976c:294) (Erythroneura), new combination Erythridula juglandacea (Ross \& DeLong, 1953a:78) (Erythroneura), new combination Erythridula juglandis (Knull \& Auten, 1938a:532) (Erythroneura), new combination Erythridula juncea (Beamer, 1937a:10) (Erythroneura), new combination Erythridula kanensis (Hepner, 1976d:314) (Erythroneura), new combination Erythridula kanza (Robinson, 1924a:58) (Erythroneura), new combination Erythridula kennethi (Hepner, 1976c:298) (Erythroneura), new combination Erythridula kingstoniensis (Hepner, 1976b:124) (Erythroneura), new combination Erythridula lasteri (Hepner, 1977a:248) (Erythroneura), new combination Erythridula latapex (Beamer, 1930b:447) (Erythroneura), new combination Erythridula lauriphylla (Hepner, 1978a:134) (Erythroneura), new combination Erythridula lawsoniana (Baker, 1926a:347 n.nov.) (Erythroneura), new combination Erythridula leforsorum (Hepner, 1977c:360) (Erythroneura), new combination Erythridula lemnisca (McAtee, 1926c:131) (Erythroneura), new combination Erythridula leucophylla (Hepner, 1977b:55) (Erythroneura), new combination Erythridula lianae (Hepner, 1976b:125) (Erythroneura), new combination Erythridula lindleyi (Hepner, 1976a:207) (Erythroneura), new combination Erythridula lloydi (Hepner, 1977a:253) (Erythroneura), new combination Erythridula loisae (Hepner, 1978a:138) (Erythroneura), new combination Erythridula lucileae (Hepner, 1976c:299) (Erythroneura), new combination Erythridula lusoria (Van Duzee, 1924a:234) (Erythroneura), new combination Erythridula lyratae (Ross \& DeLong, 1953a:83) (Erythroneura), new combination Erythridula lyratiphylla (Hepner, 1977b:55) (Erythroneura), new combination Erythridula magnacalx (Beamer, 1930b:451) (Erythroneura), new combination Erythridula malleiformis (Beamer, 1930b:449) (Erythroneura), new combination Erythridula mansueta (Beamer, 1935a:98) (Erythroneura), new combination Erythridula martini (Hepner, 1976a:207) (Erythroneura), new combination Erythridula maryae (Hepner, 1976d:313) (Erythroneura), new combination Erythridula mccomasi (Hepner, 1978a:138) (Erythroneura), new combination Erythridula meridiana (Hepner, 1977b:53) (Erythroneura), new combination Erythridula merkli (Hepner, 1977a:253) (Erythroneura), new combination Erythridula minima (Johnson, 1935a:92) (Erythroneura), new combination Erythridula minuta (Johnson, 1935a:93) (Erythroneura), new combination Erythridula mitlini (Hepner, 1977a:250) (Erythroneura), new combination Erythridula modica (Beamer, 1930b:448) (Erythroneura), new combination Erythridula morelandi (Hepner, 1976d:316) (Erythroneura), new combination Erythridula morrisi (Hepner, 1977a:251) (Erythroneura), new combination Erythridula nava (Beamer, 1935a:102) (Erythroneura), new combination 
Erythridula navoides (Hepner, 1977b:50) (Erythroneura), new combination Erythridula nebekeri (Hepner, 1976b:120) (Erythroneura), new combination Erythridula neeli (Hepner, 1976b:121) (Erythroneura), new combination Erythridula newtonensis (Hepner, 1978a:133) (Erythroneura), new combination Erythridula nigriphylla (Hepner, 1977b:54) (Erythroneura), new combination Erythridula nitida (Beamer, 1935a:103) (Erythroneura), new combination Erythridula noeva (Gillette, 1898a:757) (Typhlocyba), new combination Erythridula noevoides (Ross \& DeLong, 1953a:83) (Erythroneura), new combination Erythridula nondescripta (Johnson, 1935a:92) (Erythroneura), new combination Erythridula normanti (Hepner, 1976b:120) (Erythroneura), new combination Erythridula obliqua (Say, 1825a:342) (Tettigonia), new combination Erythridula obvia (Beamer, 1930b:439) (Erythroneura), new combination Erythridula odettae (Hepner, 1977c:362) (Erythroneura), new combination Erythridula ohioensis (Knull, 1945b:108) (Erythroneura), new combination Erythridula pagodifoliae (Hepner, 1977b:52) (Erythroneura), new combination Erythridula paigeae (Hepner, 1976a:208) (Erythroneura), new combination Erythridula paramera (Hepner, 1978a:137) (Erythroneura), new combination Erythridula parrotti (Hepner, 1977a:251) (Erythroneura), new combination Erythridula parsonsi (Hepner, 1976c:295) (Erythroneura), new combination Erythridula parvispicata (Beamer, 1930b:435) (Erythroneura), new combination Erythridula patricki (Hepner, 1976a:208) (Erythroneura), new combination Erythridula pecanae (Hepner, 1976c:297) (Erythroneura), new combination Erythridula penelutea (Beamer, 1930b:427) (Erythroneura), new combination Erythridula penenoeva (Beamer, 1930b:438) (Erythroneura), new combination Erythridula penetura (Hepner, 1978a:135) (Erythroneura), new combination Erythridula penobliqua (Beamer, 1930b:453) (Erythroneura), new combination Erythridula perita (Beamer, 1935a:99) (Erythroneura), new combination Erythridula pfrimmeri (Hepner, 1977b:49) (Erythroneura), new combination Erythridula phelliphylla (Hepner, 1977b:54) (Erythroneura), new combination Erythridula pietersi (Hepner, 1978a:137) (Erythroneura), new combination Erythridula plena (Beamer, 1930b:442) (Erythroneura), new combination Erythridula ponderosa (Auten \& Johnson, 1936a:62) (Erythroneura), new combination Erythridula praecisa (Knull, 1946a:46) (Erythroneura), new combination Erythridula pura (Knull, 1954d:38) (Erythroneura), new combination Erythridula quadrata (Beamer, 1930b:435) (Erythroneura), new combination Erythridula quadratoides (Hepner, 1977b:51) (Erythroneura), new combination Erythridula repleta (Johnson, 1935a:78) (Erythroneura), new combination Erythridula rhodedendronae (Hepner, 1978a:134) (Erythroneura), new combination Erythridula rolandi (Hepner, 1977c:364) (Erythroneura), new combination Erythridula rosenkranzi (Hepner, 1977a:254) (Erythroneura), new combination Erythridula rubens (Beamer, 1930b:439) (Erythroneura), new combination Erythridula rubiphylla (Hepner, 1977b:52) (Erythroneura), new combination Erythridula rubrataeniensis (Beamer, 1930b:440) (Erythroneura), new combination Erythridula rubroscuta (Gillette, 1898a:755) (Typhlocyba), new combination Erythridula rubrotincta (Johnson, 1935a:91) (Erythroneura), new combination Erythridula rufostigmosa (Beamer, 1930b:429) (Erythroneura), new combination Erythridula rugosae (Ross \& DeLong, 1953a:80) (Erythroneura), new combination Erythridula sabita (Sinha \& Beamer, 1954a:105) (Erythroneura), new combination Erythridula sagittata (Beamer, 1930b:440) (Erythroneura), new combination Erythridula saileri (Hepner, 1977a:253) (Erythroneura), new combination Erythridula salmoides (Ross \& DeLong, 1953a:83) (Erythroneura), new combination Erythridula schusteri (Hepner, 1976b:120) (Erythroneura), new combination Erythridula scissa (Beamer, 1930b:448) (Erythroneura), new combination Erythridula scytha (Auten \& Johnson, 1936a:61) (Erythroneura), new combination 
Erythridula shanklandi (Hepner, 1978a:137) (Erythroneura), new combination Erythridula sikorowskii (Hepner, 1976a:210) (Erythroneura), new combination Erythridula similalis (Ross \& DeLong, 1953a:82) (Erythroneura), new combination Erythridula sincera (Johnson, 1935a:94) (Erythroneura), new combination Erythridula sinua (Johnson, 1935a:82) (Erythroneura), new combination Erythridula solomoni (Hepner, 1977a:251) (Erythroneura), new combination Erythridula spatulata (Beamer, 1930b:444) (Erythroneura), new combination Erythridula spearca (Johnson \& Auten, 1936a:818 n.nov.) (Erythroneura), new combination Erythridula stolata (McAtee, 1920a:279) (Erythroneura), new combination Erythridula stulta (Auten \& Johnson, 1936a:64) (Erythroneura), new combination Erythridula stylata (Johnson, 1935a:78) (Erythroneura), new combination Erythridula styraxae (Hepner, 1976c:300) (Erythroneura), new combination Erythridula tenebrosa (Knull, 1946a:48) (Erythroneura), new combination Erythridula tenuispica (Beamer, 1930b:444) (Erythroneura), new combination Erythridula tietzi (Ross \& DeLong, 1953a:85) (Erythroneura), new combination Erythridula tolerata (Knull, 1951c:180) (Erythroneura), new combination Erythridula tomaneki (Hepner, 1977c:359) (Erythroneura), new combination Erythridula tomentosae (Hepner, 1976c:297) (Erythroneura), new combination Erythridula torva (Beamer, 1935a:98) (Erythroneura), new combination Erythridula tridenoides (Hepner, 1976d:312) (Erythroneura), new combination Erythridula tridens (Beamer, 1930b:450) (Erythroneura), new combination Erythridula tura (Ross \& DeLong, 1953a:84) (Erythroneura), new combination Erythridula turoides (Hepner, 1978a:135) (Erythroneura), new combination Erythridula ulmalatae (Ross \& DeLong, 1953a:80) (Erythroneura), new combination Erythridula ulmarubrae (Hepner, 1976d:313) (Erythroneura), new combination Erythridula ulmosa (Ross \& DeLong, 1953a:78) (Erythroneura), new combination Erythridula unicuspidis (Beamer, 1930b:452) (Erythroneura), new combination Erythridula uniforma (Hepner, 1976d:315) (Erythroneura), new combination Erythridula varia (McAtee, 1920a:287) (Erythroneura), new combination Erythridula vartyi (Hepner, 1977b:51) (Erythroneura), new combination Erythridula velutinae (Hepner, 1977b:51) (Erythroneura), new combination Erythridula verae (Hepner, 1978a:138) (Erythroneura), new combination Erythridula verdana (Ross \& DeLong, 1953a:81) (Erythroneura), new combination Erythridula victorialis (Knull, 1946a:49) (Erythroneura), new combination Erythridula vierii (Hepner, 1976b:125) (Erythroneura), new combination Erythridula vinaria (Beamer, 1930b:426) (Erythroneura), new combination Erythridula volucris (Beamer, 1930a:445) (Erythroneura), new combination Erythridula wandae (Hepner, 1976c:299) (Erythroneura), new combination Erythridula whitti (Hepner, 1976b:123) (Erythroneura), new combination Erythridula wysongi (Ross \& DeLong, 1953a:84) (Erythroneura), new combination Erythridula zephyr (Ross \& DeLong, 1953a:84) (Erythroneura), new combination 


\section{Erythroneura Fitch}

Erythroneura Fitch, 1851a:62 (type species: E. tricincta Fitch)

Erythroneura (Erythroneura) Young 1952:79

Diagnosis. Species of the genus may be recognized by the following combination of characters: ground color usually pale, dorsum frequently with bold pattern consisting of oblique lines, transverse bands, or both (Fig. 2-B); forewing usually with dark spot near base of inner apical cell, another near apex of second apical cell, and another on costal margin adjacent to brochosome field; $\mathrm{CuA}$ extremely short or absent, base of inner apical cell transverse (Fig. 1-B); male pygofer with dorsal appendage immovably fused, C-shaped (ventral arm of $\mathrm{C}$ branched in some species), without additional short internal process at base, ventral appendage absent, ventrolateral setae stout and conspicuous (Fig. 4-B); style apex with three angulate projections; aedeagus usually with elongate paired basal processes, often with pair of broad flangelike lateral processes distally, denticuli usually indistinct or absent.

Distribution. Temperate North America (one species, E. elegantula (Osborn), recorded from Panama, an apparent introduction from the United States).

Notes. The included species of Erythroneura (including E. calycula McAtee, which is nearly identical to type species E. tricincta for the included characters) were consistently recovered as a monophyletic group in the phylogenetic analysis. The genus is here restricted to equal the definition of the nominotypical subgenus of Erythroneura given by Young (1952), which currently comprises 52 valid species. The other three subgenera of Erythroneura recognized by Young are here elevated to generic status (see above and below). Most Old World species previously placed in Erythroneura have been moved to other genera, but those remaining are here considered species incertae sedis (Appendix E).

\section{Included species:}

Erythroneura aclys McAtee, 1920a:290

Erythroneura acuticephala Robinson, 1924a:61

Erythroneura amanda McAtee, 1920a:319

Erythroneura ancora Beamer, 1929b:122

Erythroneura anfracta Beamer, 1929b:123

Erythroneura aza Robinson, 1924c:291

Erythroneura beameri Robinson, 1924a:61

Erythroneura bidens McAtee, 1924c:39

Erythroneura bistrata McAtee, 1920a:305

Erythroneura caetra McAtee, 1924d:130

Erythroneura calycula McAtee, 1920a:308

Erythroneura cancellata McAtee, 1920a:320

Erythroneura coloradensis (Gillette, 1892a:16) (Typhlocyba)

Erythroneura comes (Say, 1825a:343) (Tettigonia)

Erythroneura compta McAtee, 1920a:318

Erythroneura corni Robinson, 1924a:60

Erythroneura corona McAtee, 1920a:304

Erythroneura cymbium McAtee, 1920a:310

Erythroneura delicata McAtee, 1920a:317

Erythroneura diva McAtee, 1920a:308

Erythroneura elegans McAtee, 1920a:315

Erythroneura elegantula Osborn, 1928a:289

Erythroneura festiva Beamer, 1938a:290

Erythroneura fiduciaria Knull, 1951b:170

Erythroneura fraxa Robinson, 1924c:292

Erythroneura gilensis Beamer, 1929b:123 
Erythroneura infuscata (Gillette, 1898a:764) (Typhlocyba)

Erythroneura integra McAtee, 1920a:309

Erythroneura kanwakae Robinson, 1924c:292

Erythroneura kennedyi Knull, 1945b:109

Erythroneura maritima Hamilton, in Hamilton \& Langor 1987a:679

Erythroneura noncincta Johnson, 1934a:261

Erythroneura nudata McAtee, 1920a:316

Erythroneura octonotata Walsh, 1862a:149

Erythroneura omaska Robinson, 1924a:62

Erythroneura ontari Robinson, 1924a:60

Erythroneura palimpsesta McAtee, 1924e:43

Erythroneura pontifex McAtee, 1926c:136

Erythroneura prima Beamer, 1938a:264

Erythroneura prosata Johnson, 1935a:108

Erythroneura reflecta McAtee, 1924e:43

Erythroneura repetita McAtee, 1926c:131

Erythroneura rosa Robinson, 1924a:58

Erythroneura rubra (Gillette, 1898a:764) (Typhlocyba)

Erythroneura rubrella McAtee, 1920a:316

Erythroneura tacita Beamer, 1938a:293

Erythroneura tricincta Fitch, 1851a:63

Erythroneura vaga Johnson, 1934a:260

Erythroneura vagabunda Knull, 1945b:109

Erythroneura vitifex Fitch, 1856a:392

Erythroneura vitis (Harris, 1831a:43) (Tettigonia)

Erythroneura ziczac Walsh, 1862a:149

\section{Eratoneura Young, new status}

\section{Erythroneura (Eratoneura) Young 1952:84 (type species: Erythroneura dira Beamer)}

Diagnosis. Closely resembling Erythroneura, but differing as follows: forewing (Figs. 2-C, 3-B, C) with zigzag pattern usually broken, brown spot of inner apical cell more distinct, second apical cell without dark spot distally, length of vein $\mathrm{CuA}$ variable interspecifically; pygofer appendage simple or branched (Fig. 4-C), but not C-shaped, with separate basal internal projection; aedeagal shaft usually without paired basal processes or distal flanges and usually conspicuously denticulate.

Distribution. Temperate North America.

Notes. Although the phylogenetic analysis re- covered Eratoneura (including E. ligata (McAtee), which is nearly identical to type species $E$. dira for the included characters) as paraphyletic with respect to Erythroneura, more detailed analyses, including a much larger taxon sample, will be needed to assess more accurately the phylogenetic status of these two genera. Many aspects of the coloration, pygofer structure, and genital morphology of these genera are similar, but all species of Eratoneura thus far examined have a unique fingerlike internal process at the base of the pygofer appendage that is absent in all examined species of Erythroneura. This possible synapomorphy of Eratoneura was outweighed by more homoplastic characters in the analysis. Eratoneura currently comprises 308 species. A specimen-level database and interactive key to species are available on-line at http://ctap.inhs.uiuc.edu/dmitriev/3i_keys.asp.

\section{Included species:}

Eratoneura abjecta (Beamer, 1931d:288) (Erythroneura), new combination Eratoneura acantha (Ross \& DeLong, 1950a:296) (Erythroneura), new combination Eratoneura accicurta (Hepner, 1966d:106) (Erythroneura), new combination Eratoneura accita (Knull, 1954b:170) (Erythroneura), new combination 
Eratoneura accola (McAtee, 1920a:299) (Erythroneura), new combination Eratoneura acericola (Ross \& DeLong, 1953a:88) (Erythroneura), new combination Eratoneura aculeata (Beamer, 1932g:161) (Erythroneura), new combination Eratoneura adunca (Beamer, 1932c:46) (Erythroneura), new combination Eratoneura aesculi (Beamer, 1932c:46) (Erythroneura), new combination Eratoneura affinis (Fitch, 1851a:63) (Erythroneura), new combination Eratoneura albiquera (Hepner, 1967b:62) (Erythroneura), new combination Eratoneura alevra (Ross, 1956a:89) (Erythroneura), new combination Eratoneura alicia (Ross, 1957a:185) (Erythroneura), new combination Eratoneura allecta (McAtee, 1920a:302) (Erythroneura), new combination Eratoneura alloplana (Ross, 1956a:88) (Erythroneura), new combination Eratoneura alveyi (Hepner, 1972c:269) (Erythroneura), new combination Eratoneura amboyensis (Hepner, 1972a:431) (Erythroneura), new combination Eratoneura amethica (Ross, 1957a:186) (Erythroneura), new combination Eratoneura andersoni (Beamer, 1932e:86) (Erythroneura), new combination Eratoneura anseri (Hepner, 1966d:103) (Erythroneura), new combination Eratoneura ardens (McAtee, 1920a:299) (Erythroneura), new combination Eratoneura arenosa (Ross \& DeLong, 1950a:295) (Erythroneura), new combination Eratoneura arneri (Hepner, 1969a:130) (Erythroneura), new combination Eratoneura arpegia (Ross, 1957a:188) (Erythroneura), new combination Eratoneura arta (Beamer, 1931d:287) (Erythroneura), new combination Eratoneura asymmetra (Hepner, 1973a:186) (Erythroneura), new combination Eratoneura atkinsoni (Hepner, 1972c:270) (Erythroneura), new combination Eratoneura bainteri (Hepner, 1972c:271) (Erythroneura), new combination Eratoneura ballista (Beamer, 1932e:84) (Erythroneura), new combination Eratoneura basilaris (Say, 1825a:344) (Tettigonia), new combination Eratoneura beeri (Hepner, 1972c:272) (Erythroneura), new combination Eratoneura bella (McAtee, 1920a:300) (Erythroneura), new combination Eratoneura bicurvata (Hepner, 1973a:184) (Erythroneura), new combination Eratoneura bifida (Beamer, 1931a:134) (Erythroneura), new combination Eratoneura bigemina (McAtee, 1920a:300) (Erythroneura), new combination Eratoneura billi (Hepner, 1966a:88) (Erythroneura), new combination Eratoneura biramosa (Beamer, 1941a:18) (Erythroneura), new combination Eratoneura bispinosa (Beamer, 1931b:241) (Erythroneura), new combination Eratoneura blockeri (Hepner, 1967b:72) (Erythroneura), new combination Eratoneura brazzeli (Hepner, 1966d:106) (Erythroneura), new combination Eratoneura brendae (Hepner, 1967a:21) (Erythroneura), new combination Eratoneura brevipes (Beamer, 1931a:133) (Erythroneura), new combination Eratoneura brooki (Hepner, 1969a:126) (Erythroneura), new combination Eratoneura brucensis (Hepner, 1975a:6) (Erythroneura), new combination Eratoneura byersi (Hepner, 1972c:271) (Erythroneura), new combination Eratoneura caddoensis (Hepner, 1966b:100) (Erythroneura), new combination Eratoneura calamitosa (Beamer, 1931b:241) (Erythroneura), new combination Eratoneura californica (Beamer, 1932f:143 n.nov.) (Erythroneura), new combination Eratoneura callisoga (Ross, 1956a:86) (Erythroneura), new combination Eratoneura campora (Robinson, 1924a:59) (Erythroneura), new combination Eratoneura carmini (Beamer, 1929b:121) (Erythroneura), new combination Eratoneura caverna (Hepner, 1967a:23) (Erythroneura), new combination Eratoneura cavipierra (Hepner, 1967b:64) (Erythroneura), new combination Eratoneura cera (Hepner, 1966d:105) (Erythroneura), new combination Eratoneura certa (Beamer, 1932g:159) (Erythroneura), new combination Eratoneura chehawensis (Hepner, 1966b:100) (Erythroneura), new combination Eratoneura citrosa (Ross, 1956a:86) (Erythroneura), new combination Eratoneura clara (Beamer, 1932g:161) (Erythroneura), new combination 
Eratoneura claroides (Hepner, 1967a:17) (Erythroneura), new combination Eratoneura clavipes (Beamer, 1931c:269) (Erythroneura), new combination Eratoneura codyi (Hepner, 1966d:101) (Erythroneura), new combination Eratoneura colmeri (Hepner, 1969a:132) (Erythroneura), new combination Eratoneura colvardi (Hepner, 1966b:95) (Erythroneura), new combination Eratoneura combesi (Hepner, 1972c:267) (Erythroneura), new combination Eratoneura comoides (Ross \& DeLong, 1953a:90) (Erythroneura), new combination Eratoneura compressa (Knull \& Auten, 1937a:573) (Erythroneura), new combination Eratoneura concisa (Beamer, 1931d:286) (Erythroneura), new combination Eratoneura confirmata (McAtee, 1924c:37) (Erythroneura), new combination Eratoneura consueta (Beamer, 1932d:71) (Erythroneura), new combination Eratoneura continua (Knull \& Auten, 1937a:578) (Erythroneura), new combination Eratoneura contracta (Beamer, 1931a:130) (Erythroneura), new combination Eratoneura corylorubra (Knull, 1945b:108) (Erythroneura), new combination Eratoneura coxi (Ross \& DeLong, 1950a:295) (Erythroneura), new combination Eratoneura crinita (Beamer, 1932e:85) (Erythroneura), new combination Eratoneura cristata (Knull, 1951b:174) (Erythroneura), new combination Eratoneura cunninghami (Hepner, 1966c:3) (Erythroneura), new combination Eratoneura curta (Beamer, 1932e:86) (Erythroneura), new combination Eratoneura curtoides (Hepner, 1972c:272) (Erythroneura), new combination Eratoneura curvata (Beamer, 1931a:132) (Erythroneura), new combination Eratoneura custeri (Hepner, 1966a:89) (Erythroneura), new combination Eratoneura daltonorum (Hepner, 1966a:81) (Erythroneura), new combination Eratoneura deformata (Hepner, 1966a:85) (Erythroneura), new combination Eratoneura deklei (Hepner, 1967a:20) (Erythroneura), new combination Eratoneura delongi (Knull \& Auten, 1937a:574) (Erythroneura), new combination Eratoneura denmarki (Hepner, 1967a:20) (Erythroneura), new combination Eratoneura dimidiata (Knull, 1949a:122) (Erythroneura), new combination Eratoneura dira (Beamer, 1931d:286) (Erythroneura), new combination Eratoneura direpta (Knull, 1949a:125) (Erythroneura), new combination Eratoneura distincta (Knull \& Auten, 1937a:572) (Erythroneura), new combination Eratoneura doeringae (Hepner, 1975a:7) (Erythroneura), new combination Eratoneura douglasi (Hepner, 1967b:60) (Erythroneura), new combination Eratoneura dumosa (Beamer, 1932a:13) (Erythroneura), new combination Eratoneura dura (Knull, 1954b:170) (Erythroneura), new combination Eratoneura econa (Ross, 1957a:184) (Erythroneura), new combination Eratoneura edeni (Hepner, 1967a:20) (Erythroneura), new combination Eratoneura ellisi (Hepner, 1969a:129) (Erythroneura), new combination Eratoneura eтquи (Ross \& DeLong, 1953a:88) (Erythroneura), new combination Eratoneura era (McAtee, 1920a:299) (Erythroneura), new combination Eratoneura eversi (Ross \& DeLong, 1953a:88) (Erythroneura), new combination Eratoneura externa (Beamer, 1931d:289) (Erythroneura), new combination Eratoneura facota (Beamer, 1932d:70) (Erythroneura), new combination Eratoneura fagusae (Hepner, 1967a:22) (Erythroneura), new combination Eratoneura fausta (Knull, 1951b:172) (Erythroneura), new combination Eratoneura fergersoni (Hepner, 1969a:130) (Erythroneura), new combination Eratoneura ferrosa (Hepner, 1966a:83) (Erythroneura), new combination Eratoneura firma (Beamer, 1932a:12) (Erythroneura), new combination Eratoneura flexibilis (Knull, 1949a:122) (Erythroneura), new combination Eratoneura forfex (Beamer, 1932e:82) (Erythroneura), new combination Eratoneura freytagi (Hepner, 1966a:83) (Erythroneura), new combination Eratoneura fulleri (Hepner, 1967a:19) (Erythroneura), new combination Eratoneura gemina (McAtee, 1920a:301) (Erythroneura), new combination Eratoneura gemoides (Ross, 1953b:190) (Erythroneura), new combination 
Eratoneura geronimoi (Knull, 1945b:108) (Erythroneura), new combination Eratoneura gilesi (Hepner, 1966b:97) (Erythroneura), new combination Eratoneura gillettei (Beamer, 1931a:128) (Erythroneura), new combination Eratoneura glicilla (Ross, 1956a:86) (Erythroneura), new combination Eratoneura greeni (Hepner, 1969a:127) (Erythroneura), new combination Eratoneura guicei (Hepner, 1972c:269) (Erythroneura), new combination Eratoneura hamneri (Hepner, 1969a:127) (Erythroneura), new combination Eratoneura harnedi (Hepner, 1966c:5) (Erythroneura), new combination Eratoneura harpola (Ross, 1956a:85) (Erythroneura), new combination Eratoneura hartii (Gillette, 1898a:754) (Typhlocyba), new combination Eratoneura havana (Ross \& DeLong, 1953a:90) (Erythroneura), new combination Eratoneura haysensis (Hepner, 1966a:79) (Erythroneura), new combination Eratoneura hendersoni (Hepner, 1967a:21) (Erythroneura), new combination Eratoneura hepneri (Dmitriev \& Dietrich, 2006a:38 n.nov.) (Erythroneura), new combination Eratoneura hibernia (Hepner, 1966a:83) (Erythroneura), new combination Eratoneura hutchinsi (Hepner, 1966c:2) (Erythroneura), new combination Eratoneura hyalina (Knull \& Auten, 1937a:575) (Erythroneura), new combination Eratoneura hymac (Robinson, 1924a:60) (Erythroneura), new combination Eratoneura hymettana (Knull, 1949a:124) (Erythroneura), new combination Eratoneura igella (Ross \& DeLong, 1950a:295) (Erythroneura), new combination Eratoneura imbricariae (Ross \& DeLong, 1953a:86) (Erythroneura), new combination Eratoneura immota (Beamer, 1932a:16) (Erythroneura), new combination Eratoneura impar (Beamer, 1931a:133) (Erythroneura), new combination Eratoneura incondita (Beamer, 1932a:16) (Erythroneura), new combination Eratoneura inepta (Beamer, 1932g:162) (Erythroneura), new combination Eratoneura ingrata (Beamer, 1932g:160) (Erythroneura), new combination Eratoneura inksana (Knull, 1954b:170) (Erythroneura), new combination Eratoneura interna (Beamer, 1931d:285) (Erythroneura), new combination Eratoneura johnsoni (Hepner, 1972a:432) (Erythroneura), new combination Eratoneura kansana (Baker, 1925b:537 n.nov.) (Erythroneura), new combination Eratoneura kirki (Hepner, 1967b:64) (Erythroneura), new combination Eratoneura knighti (Beamer, 1932e:87) (Erythroneura), new combination Eratoneura knullae (Ross, 1953b:192) (Erythroneura), new combination Eratoneura krameri (Hepner, 1967b:67) (Erythroneura), new combination Eratoneura kuiterti (Hepner, 1967a:17) (Erythroneura), new combination Eratoneura lamucata (Ross \& DeLong, 1953a:88) (Erythroneura), new combination Eratoneura larryi (Hepner, 1966a:89) (Erythroneura), new combination Eratoneura lata (Beamer, 1932e:86) (Erythroneura), new combination Eratoneura lawsoni (Robinson, 1924a:59) (Erythroneura), new combination Eratoneura lenta (Beamer, 1932e:82) (Erythroneura), new combination Eratoneura levecki (Hepner, 1966b:99) (Erythroneura), new combination Eratoneura ligata (McAtee, 1920a:301) (Erythroneura), new combination Eratoneura lillianae (Hepner, 1966a:89) (Erythroneura), new combination Eratoneura linea (Beamer, 1932c:47) (Erythroneura), new combination Eratoneura longa (Knull, 1955a:245) (Erythroneura), new combination Eratoneura longifurca (Hepner, 1966c:3) (Erythroneura), new combination Eratoneura loriae (Hepner, 1967b:70) (Erythroneura), new combination Eratoneura lucora (Hepner, 1967a:24) (Erythroneura), new combination Eratoneura luculenta (Knull, 1949a:124) (Erythroneura), new combination Eratoneura lucyae (Hepner, 1966a:81) (Erythroneura), new combination Eratoneura lunata (McAtee, 1924e:41) (Erythroneura), new combination Eratoneura lundi (Hepner, 1967a:22) (Erythroneura), new combination Eratoneura lyriquera (Hepner, 1967b:61) (Erythroneura), new combination Eratoneura macra (Beamer, 1932a:15) (Erythroneura), new combination 
Eratoneura maculata (Gillette, 1898a:764) (Typhlocyba), new combination Eratoneura maga (Knull, 1951b:170) (Erythroneura), new combination Eratoneura malaca (Knull, 1949a:126) (Erythroneura), new combination Eratoneura manus (Beamer, 1932e:83) (Erythroneura), new combination Eratoneura marilandicae (Ross, 1957a:183) (Erythroneura), new combination Eratoneura mariquera (Hepner, 1973a:186) (Erythroneura), new combination Eratoneura marra (Beamer, 1932g:160) (Erythroneura), new combination Eratoneura masonae (Knull, 1954b:171) (Erythroneura), new combination Eratoneura maxwelli (Hepner, 1972b:218) (Erythroneura), new combination Eratoneura meadi (Hepner, 1966a:79) (Erythroneura), new combination Eratoneura mensa (Beamer, 1931d:287) (Erythroneura), new combination Eratoneura metopia (Ross, 1957a:185) (Erythroneura), new combination Eratoneura micheneri (Hepner, 1972c:271) (Erythroneura), new combination Eratoneura millsi (Ross \& DeLong, 1950a:291) (Erythroneura), new combination Eratoneura mimica (Ross, 1957a:190) (Erythroneura), new combination Eratoneura minor (Beamer, 1932e:84) (Erythroneura), new combination Eratoneura mira (Beamer, 1932c:45) (Erythroneura), new combination Eratoneura mirifica (Beamer, 1932d:159) (Erythroneura), new combination Eratoneura misera (Beamer, 1932g:158) (Erythroneura), new combination Eratoneura morgani (DeLong, 1916a:104) (Typhlocyba), new combination Eratoneura natchezensis (Hepner, 1967b:70) (Erythroneura), new combination Eratoneura nevadensis (Beamer, 1932d:72) (Erythroneura), new combination Eratoneura nielsoni (Hepner, 1967b:62) (Erythroneura), new combination Eratoneura nigriquera (Hepner, 1967b:60) (Erythroneura), new combination Eratoneura nigriventer (Beamer, 1931a:134) (Erythroneura), new combination Eratoneura nimia (Knull, 1954b:171) (Erythroneura), new combination Eratoneura noncuspidis (Beamer, 1931a:129) (Erythroneura), new combination Eratoneura norica (Ross, 1956a:88) (Erythroneura), new combination Eratoneura omani (Beamer, 1930a:49) (Erythroneura), new combination Eratoneura opulenta (Beamer, 1932c:48) (Erythroneura), new combination Eratoneura osborni (DeLong, 1916a:103) (Typhlocyba), new combination Eratoneura ostryae (Hepner, 1967a:23) (Erythroneura), new combination Eratoneura pallida (Knull \& Auten, 1937a:573) (Erythroneura), new combination Eratoneura paluloides (Ross, 1953b:189) (Erythroneura), new combination Eratoneura pamelae (Hepner, 1967b:67) (Erythroneura), new combination Eratoneura paraesculi (Knull, 1945b:106) (Erythroneura), new combination Eratoneura parallela (McAtee, 1924c:38) (Erythroneura), new combination Eratoneura parisensis (Hepner, 1972a:431) (Erythroneura), new combination Eratoneura parva (Beamer, 1932d:70) (Erythroneura), new combination Eratoneura parvipes (Beamer, 1931b:242) (Erythroneura), new combination Eratoneura patei (Hepner, 1967a:19) (Erythroneura), new combination Eratoneura patriciae (Hepner, 1966a:88) (Erythroneura), new combination Eratoneura patris (Ross \& DeLong, 1953a:89) (Erythroneura), new combination Eratoneura paulae (Hepner, 1966a:81) (Erythroneura), new combination Eratoneura penerostrata (Beamer, 1932e:85) (Erythroneura), new combination Eratoneura penesica (Beamer, 1931c:269) (Erythroneura), new combination Eratoneura perplexa (Knull, 1944b:123 n.nov.) (Erythroneura), new combination Eratoneura phellos (Ross \& DeLong, 1953a:90) (Erythroneura), new combination Eratoneura pitrei (Hepner, 1973a:184) (Erythroneura), new combination Eratoneura priniquera (Hepner, 1967b:68) (Erythroneura), new combination Eratoneura prolixa (Knull, 1949a:126) (Erythroneura), new combination Eratoneura propria (Beamer, 1932a:13) (Erythroneura), new combination Eratoneura protuma (Ross, 1957a:188) (Erythroneura), new combination Eratoneura pumicasta (Hepner, 1967b:72) (Erythroneura), new combination 
Eratoneura pyra (McAtee, 1924d:133) (Erythroneura), new combination Eratoneura quercalbae (Ross \& DeLong, 1950a:294) (Erythroneura), new combination Eratoneura rangifer (Ross \& DeLong, 1950a:292) (Erythroneura), new combination Eratoneura ratcliffensis (Hepner, 1966a:85) (Erythroneura), new combination Eratoneura reedi (Hepner, 1967b:61) (Erythroneura), new combination Eratoneura reiteri (Hepner, 1966a:87) (Erythroneura), new combination Eratoneura restricta (Beamer, 1932c:45) (Erythroneura), new combination Eratoneura retusa (Beamer, 1932c:48) (Erythroneura), new combination Eratoneura richardsi (Ross, 1953b:189) (Erythroneura), new combination Eratoneura robinsoni (Hepner, 1966a:89) (Erythroneura), new combination Eratoneura robusta (Knull, 1955a:245) (Erythroneura), new combination Eratoneura rossi (Hepner, 1966a:85) (Erythroneura), new combination Eratoneura rostrata (Beamer, 1931c:270) (Erythroneura), new combination Eratoneura rotunda (Beamer, 1931d:288) (Erythroneura), new combination Eratoneura rubida (Knull, 1954b:171) (Erythroneura), new combination Eratoneura rubranotata (Beamer, 1927a:30) (Erythroneura), new combination Eratoneura rubrarta (Hepner, 1967b:66) (Erythroneura), new combination Eratoneura rubraza (Robinson, 1924c:291) (Erythroneura), new combination Eratoneura rubulna (Hepner, 1972a:433) (Erythroneura), new combination Eratoneura sadleri (Hepner, 1969a:132) (Erythroneura), new combination Eratoneura sancta (Beamer, 1932a:15) (Erythroneura), new combination Eratoneura sanctaerosae (Hepner, 1967a:24) (Erythroneura), new combination Eratoneura sandersoni (Ross, 1956a:90) (Erythroneura), new combination Eratoneura schista (Knull, 1955a:246) (Erythroneura), new combination Eratoneura scobyensis (Hepner, 1966b:97) (Erythroneura), new combination Eratoneura sebringensis (Hepner, 1966b:97) (Erythroneura), new combination Eratoneura separata (Beamer, 1932a:12) (Erythroneura), new combination Eratoneura septima (Beamer, 1927a:30) (Erythroneura), new combination Eratoneura sethi (Hepner, 1966b:100) (Erythroneura), new combination Eratoneura severini (Knull, 1949a:125) (Erythroneura), new combination Eratoneura shumiquera (Hepner, 1972a:431) (Erythroneura), new combination Eratoneura siloamensis (Hepner, 1967a:22) (Erythroneura), new combination Eratoneura smithi (Ross, 1956a:90) (Erythroneura), new combination Eratoneura socia (Knull, 1954b:171) (Erythroneura), new combination Eratoneura solida (Knull, 1954b:171) (Erythroneura), new combination Eratoneura solita (Beamer, 1932a:14) (Erythroneura), new combination Eratoneura sorota (Hepner, 1975a:5) (Erythroneura), new combination Eratoneura spala (Ross \& DeLong, 1950a:294) (Erythroneura), new combination Eratoneura spinea (Knull, 1951b:172) (Erythroneura), new combination Eratoneura spinifera (Beamer, 1931b:240) (Erythroneura), new combination Eratoneura spiniterma (Hepner, 1969a:133) (Erythroneura), new combination Eratoneura staffordi (Hepner, 1966c:3) (Erythroneura), new combination Eratoneura staminea (Knull, 1954b:171) (Erythroneura), new combination Eratoneura stannardi (Hepner, 1967b:66) (Erythroneura), new combination Eratoneura starkvillensis (Hepner, 1966d:103) (Erythroneura), new combination Eratoneura stephensoni (Beamer, 1931a:130) (Erythroneura), new combination Eratoneura stoveri (Ross \& DeLong, 1950a:296) (Erythroneura), new combination Eratoneura stupkaorum (Knull, 1945b:104) (Erythroneura), new combination Eratoneura tammina (Ross \& DeLong, 1953a:86) (Erythroneura), new combination Eratoneura tantilla (Beamer, 1931d:285) (Erythroneura), new combination Eratoneura tantula (Knull, 1954b:171) (Erythroneura), new combination Eratoneura tenilla (Ross \& DeLong, 1950a:294) (Erythroneura), new combination Eratoneura tenuitas (Knull, 1954b:173) (Erythroneura), new combination Eratoneura teres (Beamer, 1931c:268) (Erythroneura), new combination 
Eratoneura tersa (Knull, 1951b:172) (Erythroneura), new combination Eratoneura teshi (Hepner, 1972c:267) (Erythroneura), new combination Eratoneura texana (Beamer, 1929b:121) (Erythroneura), new combination Eratoneura thaxtoni (Hepner, 1966c:2) (Erythroneura), new combination Eratoneura torella (Robinson, 1924b:156) (Erythroneura), new combination Eratoneura trautmanae (Knull, 1945b:104) (Erythroneura), new combination Eratoneura triangulata (Beamer, 1931b:240) (Erythroneura), new combination Eratoneura trivittata (Robinson, 1924a:59) (Erythroneura), new combination Eratoneura tumida (Knull, 1954b:173) (Erythroneura), new combination Eratoneura turgida (Beamer, 1931b:243) (Erythroneura), new combination Eratoneura unca (Knull, 1954b:174) (Erythroneura), new combination Eratoneura uncinata (Beamer, 1931b:242) (Erythroneura), new combination Eratoneura ungulata (Beamer, 1932d:69) (Erythroneura), new combination Eratoneura unica (Beamer, 1932e:83) (Erythroneura), new combination Eratoneura univittata (Robinson, 1924b:156) (Erythroneura), new combination Eratoneura usitata (Beamer, 1932a:14) (Erythroneura), new combination Eratoneura uvaldeana (Knull, 1949a:125) (Erythroneura), new combination Eratoneura vacua (Knull, 1954b:174) (Erythroneura), new combination Eratoneura valida (Knull, 1954b:174) (Erythroneura), new combination Eratoneura velox (Ross, 1953b:190) (Erythroneura), new combination Eratoneura ventura (Knull \& Auten, 1937a:577) (Erythroneura), new combination Eratoneura vinsoni (Hepner, 1969a:129) (Erythroneura), new combination Eratoneura vittata (Knull \& Auten, 1937a:577) (Erythroneura), new combination Eratoneura vulgaris (Hepner, 1975a:4) (Erythroneura), new combination Eratoneura weemsi (Hepner, 1967a:19) (Erythroneura), new combination Eratoneura whitcombi (Hepner, 1975a:6) (Erythroneura), new combination Eratoneura wilsoni (Hepner, 1966d:106) (Erythroneura), new combination Eratoneura winslowensis (Hepner, 1966a:85) (Erythroneura), new combination Eratoneura wisei (Hepner, 1966b:97) (Erythroneura), new combination Eratoneura wiyguli (Hepner, 1966d:103) (Erythroneura), new combination Eratoneura wolcottensis (Hepner, 1973a:185) (Erythroneura), new combination Eratoneura woodruffi (Hepner, 1967a:23) (Erythroneura), new combination Eratoneura youngi (Hepner, 1966a:87) (Erythroneura), new combination Eratoneura zioni (Beamer, 1932d:71) (Erythroneura), new combination

Erasmoneura Young, new status

\section{Erythroneura (Erasmoneura) Young 1952:80 (type species: Erythroneura vulnerata Fitch)}

Diagnosis. Resembling Erythroneura in having third point on male style, but differing as follows: coloration usually darker overall; head usually more strongly produced (Fig. 2-D); base of forewing inner apical cell angulate and lacking distinct brown spot (Fig. 3-D); pygofer appendage separated from margin by a basal line of weakening (Fig. 4-D); aedeagus with preatrium elongate, with pair of apical or preapical spines or processes, without elongate basal processes.
Distribution. Temperate North America; introduced into southern Europe (Duso, et al. 2005). Notes. Phylogenetic analysis of morphological characters placed the included species of Erasmoneura as monophyletic sister to the Erythroneura + Eratoneura clade, justifying the elevation of Erasmoneura to generic rank. Three species formerly included in Erasmoneu$r a$ are here removed and placed in a separate new genus, Rossmoneura, described below based on differences in coloration, the structure of the head, and the structure of the aedeagus. Erasmoneura currently comprises 12 valid species. A specimen-level database and interactive key to species are available on-line at http:// ctap.inhs.uiuc.edu/dmitriev/3i_keys.asp. 


\section{Included species:}

Erasmoneura atrata (Johnson, 1935a:97) (Erythroneura), new combination Erasmoneura bicolorata (Beamer, 1937a:11) (Erythroneura), new combination Erasmoneura bipentagona (Beamer, 1927a:31) (Erythroneura), new combination Erasmoneura caerula (Beamer, 1937a:10) (Erythroneura), new combination Erasmoneura fulmina (McAtee, 1920a:274) (Erythroneura), new combination Erasmoneura gradata (Robinson, 1924a:58) (Erythroneura), new combination Erasmoneura mixta (Beamer, 1932j:183) (Erythroneura), new combination Erasmoneura nigerrima (McAtee, 1920a:275) (Erythroneura), new combination Erasmoneura nigra (Gillette, 1898a:765) (Typhlocyba), new combination Erasmoneura rubricata (Van Duzee, 1909a:229) (Typhlocyba), new combination Erasmoneura variabilis (Beamer, 1929b:126) (Erythroneura), new combination Erasmoneura vulnerata (Fitch, 1851a:62) (Erythroneura), new combination

\section{Hymetta McAtee}

\section{Hymetta McAtee 1919:121 (type species: Tettigonia trifasciata Say)}

Diagnosis. This small, distinctive genus is characterized by the following combination of features: head pale, without dark spots on face or crown; scutellum elevated posteriorly, apex black; forewing broad, marked with numerous small irregular reddish or brownish flecks (Fig. 2-E); pygofer with dorsal appendage immov-

\section{Included species:}

Hymetta anthisma McAtee, 1919a: 123

Hymetta arizoniana Fairbairn, 1928a: 90

Hymetta balteata McAtee, 1919a: 123

Hymetta distincta Fairbairn, 1928a: 87

Hymetta kansasensis Fairbairn, 1928a: 90

Hymetta trifasciata (Say, 1825a: 343) (Tettigonia)

\section{Descriptions of New Taxa}

\section{North American Genera}

\section{Aztegina, new genus}

(Figs. 2-F; 3-F, V; 4-F; 6-A-E; 11-A, O)

Type species: Aztegina punctinota, new species, here designated.

Diagnosis. Length $3.1-3.3 \mathrm{~mm}$. Color pale yellow with brown markings; head with small median apical spot; mesonotal triangles dark brown; apex of scutellum black; forewing symmetrically marked with brown but without distinct pattern (Fig. 2-F). Head narrower than pronotum; crown angulately produced, longer medially than next to eye, coronal suture complete. Scutellum not elevated apically. Forewing (Fig. 3-F) ably fused to margin, ventral appendage absent, basolateral setal group well differentiated (Fig. 4-E); style apex footlike without third point. Distribution. Temperate North America. Notes. Phylogenetic analysis supported the monophyly of Hymetta and placed the genus as sister to Illinigina, n. gen. Hymetta currently comprises six valid species. A specimen-level database and interactive key to species are available on-line at http://ctap.inhs.uiuc.edu/ dmitriev/3i_keys.asp. with inner apical cell base oblique, length of $\mathrm{CuA}$ subequal to that of $\mathrm{CuP}$; third apical cell slender, parallel-sided; outer apical cell less than $2 \mathrm{X}$ longer than wide; anal vein moderately well delimited. Hindwing (Fig. 3-V) vein RA present; $\mathrm{m}$-cu crossvein absent, $\mathrm{CuA}$ confluent with MP for short distance. Sternite IX without median internal ridge. Pygofer (Figs. 4-F, 11-O) with broad dorsal emargination not extended to base; without oblique dorsolateral internal ridge; surface with conspicuous microtrichia distally, sparse fine setae basolaterally; basolateral setal group undifferentiated; apical lobe with group of short fine setae on mesal surface; dorsal appendage rigidly attached to pygofer margin, slender, upturned distally; ventral appendage absent; unsclerotized areas restricted to margin. First segment of anal tube with basolateral arm well developed, connected by membrane to 
pygofer just ventrad of dorsal appendage base, microtrichia inconspicuous. Subgenital plate (Fig. 6-A) with basolateral angle acute; without conspicuous dorsal fold; submarginal row with three macrosetae; marginal row with short peglike setae basally and short fine setae distally; distal lobe weakly expanded. Connective (Fig. 6-D) V-shaped, without stem or median keel. Style (Fig. 6-E) preapical lobe large, truncate; apophysis short, footlike, with imbricate texture. Aedeagus (Figs. 6-B, C) without preatrium; dorsal apodeme long, narrow, weakly expanded dorsally, apex T-shaped in posterior view, connections to pygofer membranous; shaft elongate with short paired apical processes; gonopore subapical on posterior surface.

Distribution. Mexico.

Notes. The genus name, which is feminine, combines "Aztec" the indigenous people who inhabited the vicinity of present-day Mexico City prior to the arrival of the Spanish conquistadors, with "Zygina" a genus of Erythroneurini. The genus is based on a single new species recorded from Mexico. In the phylogenetic analysis, this species was consistently placed as sister to a clade comprising the New World genera Mexigina, Neozygina, Hepzygina, and Zyginama.

\section{Included species:}

Aztegina punctinota, n. sp.

\section{Aztegina punctinota, new species}

\section{Type locality: Distrito Federal, Mexico} Diagnosis. Length of male $3.1 \mathrm{~mm}$, female $3.3 \mathrm{~mm}$. Ground color yellow-green; crown midline, two large sublateral pronotal spots, two maculae on either end of forewing brochosome field, and mesosternum dark brown. Forewing with diffuse transcommisural diamond-shaped brown mark in basal half, distal third infused with brown; metathorax except legs and wings bright orange. Male abdominal $2 \mathrm{~S}$ apodemes relatively narrow, extended to posterior margin of sternite III (Fig. 11-A). Genital capsule, connective, and styles as described for genus; aedeagus with shaft in posterior view (Fig. 6-C) bearing pair of rounded lateroapical lobes; pair of slender processes arising distad of gonopore extended anterolaterad.

Material examined. Holotype $\sigma^{\top}$ : MEXICO: DF: rt. 95 km 41 S. Mexico City, 2970m,
N19.12496, W99.19440, 18-X-2005, C. H. Dietrich, MX05-03-2, sweeping; $6 \sigma^{7} \sigma^{7}$ and 4 우 우 paratypes, same data [INHS]. Other material: 1 기, MEXICO: DF: Mexico City, 13IX-1939, D. M. DeLong [INHS].

\section{Hepzygina, new genus}

(Figs. 2-G; 3-G; 4-G; 6-F, G)

Type species: Erythroneura milleri Beamer, here designated

Diagnosis. Length $2.3-3 \mathrm{~mm}$. Color pale overall with various orange and brown markings; head with or without small apical brown spot and pair of indistinct preapical orange or brown spots on crown (Fig. 2-G). Head slightly narrower than pronotum; crown angulately produced, slightly longer medially than next to eyes, coronal suture complete. Wing venation (Fig. 3-G) as in Aztegina. Sternite IX with median internal ridge anteriorly. Pygofer (Fig. 4-G) broadly emarginate dorsally to near base; without oblique dorsolateral internal ridge; surface with distinct microtrichia and scattered fine setae; basolateral setal group well developed with several moderately large setae; apical lobe with group of short slender setae on mesal surface; dorsal appendage elongate, straight or slightly sinuate, rigidly attached to pygofer margin; ventral appendage absent; posteroventral area weakly sclerotized. First segment of anal tube with basolateral arm well developed, straight, articulated near pygofer dorsal margin; microtrichia inconspicuous. Subgenital plate with basolateral angle acute; without conspicuous dorsal fold; submarginal row with three macrosetae; marginal row continuous from base to apex, with microsetae short and slender; distal lobe not expanded. Connective U-shaped; stem broad, bifid, depressed, overlapping base of aedeagus. Style preapical lobe very large; apophysis short, smooth, truncate, with two points. Aedeagus (Figs. 6-F, G) with preatrium short; dorsal apodeme short, compressed, expanded anterad in lateral view, with pair of posterolateral lobes, connections to pygofer membranous; shaft compressed with elongate recurved median ventral process arising near base, paired distal processes absent.

Distribution. Southwestern USA and Mexico. Notes. Phylogenetic analysis recovered the two included species as a monophyletic sister group to Zyginama, n. gen. The genus name, 
which is feminine, was formed by combining the first syllable of the surname of Dr. Leon Hepner, who described many of the New World Erythroneurini, with Zygina, the genus in which the type species was previously placed. This genus is described based on two species previously included by Young (1952) in the "Ritana group" of Zygina. Examination of the female holotype of Erythroneura tergemina Van Duzee
(California Academy of Sciences) reveals that it is a synonym of $H$. aprica (McAtee). Both names were published in the same year, but the latter appears to have priority. A few Mexican specimens of this genus in the INHS collection differ somewhat from the previously described species in the structure of the aedeagus and may represent morphological variants or undescribed species.

\section{Included species: \\ Hepzygina aprica (McAtee, 1924d:132) (Erythroneura), new combination \\ Erythroneura tergemina Van Duzee, 1924a:235, new synonym \\ Hepzygina milleri (Beamer, 1929b:125 ) (Erythroneura), new combination}

\section{Illinigina, new genus}

(Figs. 2-H; 3-H, W; 4-H, 6-I-L)

\section{Type species: Erythroneura illinoiensis Gil- lette, here designated.}

Diagnosis. Length $2.8-3.2 \mathrm{~mm}$. Overall color pale yellow, with median red or brown spots on pronotum, scutellum, and sometimes crown; forewing with few symmetrical brown spots or maculae in basal half, apical cells unmarked (Fig. 2-H). Head slightly narrower than pronotum; crown angulately produced, longer medially than next to eye. Scutellum not elevated apically. Forewing (Fig. 3-H) with inner apical cell base oblique, length of $\mathrm{CuA}$ subequal to that of $\mathrm{CuP}$; third apical cell relatively broad, slightly widened distally; outer apical cell approximately $2 \mathrm{X}$ longer than wide; anal vein obsolete. Hindwing (Fig. 3-W) with vein RA present; m-cu very short, $\mathrm{CuA}$ and MP nearly touching at one point. Sternite IX with median longitudinal internal ridge anteriorly. Pygofer (Fig. 4-H) with narrow dorsal emargination extended to base; dorsolateral oblique internal ridge present; surface sparsely clothed with fine setae, microtrichia inconspicuous; basolateral setal group present but poorly differentiated; apical lobe with group of short slender setae on mesal surface; dorsal appendage short, tapered, upturned, movably articulated to dorsal margin of pygofer, connected to dorsal apodeme of aedeagus by well-sclerotized ligament (Figs. 6-I, J); ventral appendage absent; unsclerotized areas restricted to ventral margin. First segment of anal tube with basolateral arm poorly developed, microtrichia inconspicuous. Subgenital plate (Fig. 4H) with basolateral angle acute; dorsal fold angulate; submarginal row with 3-4 macrosetae; marginal row with short, fine setae; distal lobe weakly expanded. Connective (Fig. 6-K) Y-shaped; stem short, compressed. Style (Fig. 6L) preapical lobe well developed, oblique; apophysis short, smooth, apex hatchetlike, with two points. Aedeagus (Figs. 6-I, J) with preatrium well developed; dorsal apodeme well developed, somewhat expanded in lateral view; shaft short, compressed, with pair of slender basal processes; gonopore terminal. Distribution. Temperate North America. Notes. The genus name, which is feminine, is derived in part from the name of its only included species, which in turn is based on the type locality. The only known species of the genus, I. illinoiensis, was sister to Hymetta in the phylogenetic analysis. These two genera have the dorsal appendage of the pygofer very similar in shape, both lack a second extension on the male style apex, and both have the scutellum apex darkly pigmented, in contrast to the rest of the scutellum. Illinigina differs from Hymetta in having the forewings more slender and lacking red flecks and transverse bands, and the scutellum not elevated posteriorly. I. illinoiensis is widespread and somewhat variable in coloration (two named color forms, regalis Beamer and spectra McAtee are considered synonyms of I. illinoiensis) but is readily distinguished from other New World Erythroneurini by the distinctive pattern of red and brown spots on the scutellum, forewing, and usually also the head and pronotum. 
Included species:

Illinigina illinoiensis (Gillette, 1898a:758) (Typhlocyba), new combination

Erythroneura illinoiensis var. regalis Beamer 1929b:125, new synonym

Erythroneura illinoiensis var. spectra McAtee 1920a:292, new synonym

\section{Mexigina, new genus}

(Figs. 1-A, D; 2-I; 3-I, X; 4-I; 6-M-P; 11-B)

Type species: Erythroneura oculata McAtee, here designated.

Diagnosis. Length $2.5-2.7 \mathrm{~mm}$. Dorsal coloration (Fig. 2-I) mostly dark brown; head pale with two pairs of preapical brown spots, one on crown, one more widely separated just dorsad of antennal pits (Fig. 1-A). Mesonotal triangles dark brown, apex of scutellum pale. Forewing (Fig. 3-I) heavily infused with fuscous, without distinct pattern; inner apical cell base oblique, length of $\mathrm{CuA}$ subequal to that of $\mathrm{CuP}$; third apical cell broadened distally; outer apical cell approximately $2 \mathrm{X}$ longer than wide; anal vein poorly delimited. Hind wing vein RA absent; $\mathrm{m}$-cu crossvein absent, CuA confluent with MP for short distance. Sternite IX with median longitudinal internal ridge anteriorly. Pygofer (Fig. 4-I) broadly emarginate to base dorsally; without dorsolateral oblique ridge; surface with distinct microtrichia, fine setae sparse; basolateral setal group well differentiated, setae somewhat enlarged; apical lobe with group of short slender setae near margin; dorsal margin

\section{Included species:}

Mexigina oculata (McAtee, 1924e:39) (Erythroneura), new combination

\section{Nelionidia, new genus}

\section{(Figs. 2-J; 3-K-M, Z; 4-K-M; 7-A-O; 1-D, $\mathrm{Q}-\mathrm{S})$}

Type species: $N$. pueblensis, new species, here designated.

Diagnosis. Length $2.5-3.3 \mathrm{~mm}$. Ground color pale yellow, variously marked with brown, head with pair of preapical brown spots on crown (Fig. 2-J). Head slightly narrower than pronotum; crown produced, slightly longer medially than next to eye, coronal suture complete. Forewing (Figs. 3-K-M) inner apical cell base angulate; $\mathrm{CuA}$ shorter than $\mathrm{CuP}$, subequal to basal segment of MP; third apical cell widened distally; outer apical cell usually strongly sclerotized but without appendage; ventral appendage well developed; membranous areas restricted to margin. First segment of anal tube with basolateral arm well developed, expanded distally, articulated to pygofer margin; microtrichia indistinct. Subgenital plate (Fig. 4-I) with basolateral angle acute; without conspicuous dorsal fold; submarginal row with 3-4 macrosetae; marginal row with setae short and slender, very sparse distally; distal lobe not expanded. Connective Ushaped; stem short, compressed into dorsal keel. Style (Fig. 6-P) with preapical lobe large, truncate; apophysis short, smooth, footlike. Aedeagus (Figs. 6-M, N) without preatrium; dorsal apodeme slender, T-shaped in posterior view, connections to pygofer membranous; shaft compressed, with pair of lamelliform apical processes; gonopore preapical on posterior surface.

Distribution. Southwestern USA and Mexico. Notes. This is the only North American erythroneurine genus known to lack a dorsal pygofer appendage. The only known species is M. oculata (McAtee), which was included by Young (1952) in the "ritana group" of Zygina. The genus name, which is feminine, refers to the primarily Mexican distribution of the type species. twice as long as wide. Hindwing (Fig. 3Z) vein RA weakly delimited; MP and CuA confluent for short distance. Sternite IX without median internal ridge. Pygofer (Figs. 4-J-M; 11-Q-S) weakly to strongly emarginate dorsally; oblique dorsolateral internal ridge weakly to strongly developed; surface with distinct microtrichia, without fine setae; basolateral setal group well delimited with several setae; apical lobe with distinct group of short slender setae on inner surface; dorsal appendage usually curved ventrad then posterad, articulated to pygofer side, anteroventral lobe connected by well-sclerotized tendon to dorsal apodeme of aedeagus; ventral appendage absent. First segment of anal tube with basolateral arm weakly sclerotized, extended to junction of aedeagal tendon 
and pygofer appendage; microtrichia inconspicuous. Subgenital plate (Figs. 4J-M) with basolateral angle obtuse; without conspicuous dorsal fold; submarginal row with three macrosetae; marginal row with microsetae short and slender, continuous from subbasal angle to near apex. Connective (Fig. 7-D) broadly U-shaped; stem short, compressed. Style (Fig. 7-C) preapical lobe prominent but not greatly enlarged; apophysis smooth, with three points or angles of various sizes. Aedeagus (Figs. 7-A, B) with preatrium short or absent; dorsal apodeme in lateral view long, narrow, compressed; shaft elongate, slender, with pair of long recurved processes apically, gonopore apical or subapical on posterior surface. Fe- male sternite VII with posterior margin acutely produced, process weakly constricted.

\section{Distribution. Mexico.}

Notes. The genus name, which is feminine, is a modification of that of the superficially similar Eremian genus Helionidia Zachvatkin, which differs in having a distinctive style and the connective with a median anterior lobe. Nelionidia is described based on four new species from Mexico and one previously described species, Erythroneura amicis Ross. The latter was not selected as the type species because its dorsal pygofer appendage differs considerably from that of other species in the genus, and because only a single specimen is known.

\section{Included species:}

Nelionidia amicis (Ross, 1965:268) (Erythroneura), new combination

Nelionidia elliptica, n. sp.

Nelionidia moyai, n. sp.

Nelionidia pallescens, n. sp.

Nelionidia pueblensis, n. sp.

\section{Key to Males of Nelionidia}

1 Dorsum nearly uniformly pale yellow except pair of brown preapical spots on crown; aedeagus without anteriorly projecting subapical spine (Fig. 7-H) .......................pallescens, n. sp. 1 ' Dorsum heavily marked with dark brown (Fig. 2-J); aedeagus with anteriorly projecting subapi-

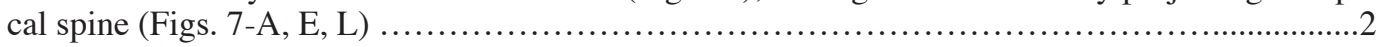

2 Pygofer with posteromedial emargination constricted and parallel-sided in basal half; style apex with third point elongate, approximately as long as distance between first and second points (Fig.

$1-\mathrm{H})$

2' Pygofer with sides of posteromedial emargination evenly tapered; style apex with third point short, much less than half distance between first and second points (Figs. 7-C, J, M)

3 Pygofer dorsal appendage bifid distally in dorsal view (Ross 1965: Fig. 33-B), falcate in lateral view; aedeagus with shaft apex in posterior view broadened and truncate with median notch (ibid: Fig. 33-A) amicis (Ross)

3' Pygofer dorsal appendage simple, extended ventrad, then bent posterad (Fig. 4-K); aedeagus with shaft apex in posterior view entire, without notch (Fig. 7-F) ..moyai, n. sp.

4 Pygofer dorsal appendage broad basally, with spinelike distal projection(Fig. 4-M); aedeagus with apex in posterior view broadly rounded (Fig. 7-M) ..........................pueblensis, n. sp. 4' Pygofer dorsal appendage evenly tapered from base to apex (Fig. 4-J); aedeagus with apex in posterior view truncate with pair of laterally directed spines (Fig. 7-B) elliptica, n. sp. 


\section{Nelionidia elliptica, new species}

(Figs. 2-J; 4-J; 7-A-D; 11-Q)

Type locality: ca. La Huerta, Jalisco, Mexico. Diagnosis. Length of male $2.7 \mathrm{~mm}$. Head pale yellow, anteclypeus and areas adjacent to clypeogenal suture brown, apex infused with orange, crown with pair of brown preapical spots well developed; pronotum margins pale, medial area with symmetrical brown markings; mesonotal triangles and mesothoracic venter dark brown; scutellum yellow infused with brown; forewing brown, with large white spots at base of clavus, base of corium, and subapically in brachial cell; costal brochosome field and veins yellow. Male abdominal $2 \mathrm{~S}$ apodemes short, extended dorsomesad, not reaching posterior margin of sternite III. Pygofer (Fig. 4-J, 11Q) with posteromedial emargination shallow, V-shaped; dorsal appendage extended ventrad, then abruptly bent posterad, distal section slightly arcuate. Aedeagus (Figs. 7-A-D) with apex in lateral view tapered, in posterior view truncate with pair of lateral projections; long paired processes arising preapically adjacent to gonopore, sinuate in lateral view, in posterior evenly curved ventrolaterad, then mesad, apices extended to near midline. Style apex (Fig. 7-A) with first and second points broad, third point short, acute, arising near midpoint between first and second points and angled toward first point. Material examined. Holotype $\sigma^{\top}$, MEXICO: Jalisco, ca. La Huerta, 480m, 19²7’47'N $104^{\circ} 39^{\prime} 13^{\prime \prime W}, 16$ Oct 2001, S. H. McKamey, et al., fogging 3-FG [INHS].

Note. The species name refers to the form of the aedeagus in posterior view.

\section{Nelionidia moyai, new species}

$$
\text { (Figs. 1-H; 3-K, Z; 4K; 7-E-G; 11-R) }
$$

Type locality: ca. La Huerta, Jalisco, Mexico. Diagnosis. Length of male $2.7 \mathrm{~mm}$, female 2.5-2.7mm. Coloration as described for $N$. elliptica. Male abdominal 2S apodemes short, extended dorsomesad, not reaching posterior margin of sternite III. Pygofer (Figs. 4-K, 11$\mathrm{R})$ with posteromedial emargination extended to base of segment, basal half parallel-sided; dorsal appendage extended ventrad, then abruptly bent posterad, distal section elongate, much longer than basal section, nearly straight in lateral view. Aedeagus (Figs. 7-D-F) with apex truncate in posterior view (but see Note below); paired processes relatively short, extended ventrolaterad in posterior view. Style (Fig. 1-H) apex with third point slender, elongate, nearly as long as distance between first and second points, arising approximately two-thirds distance between first and second point.

Material examined. Holotype $\sigma^{7}$, MEXICO: Jalisco, ca. La Huerta, 480m, 19²7'47’N $104^{\circ} 39^{\prime} 13$ 'W, 16 Oct 2001, S.H.McKamey, et al., fogging 3-FI [INHS]; 2 ठ ठ same data, except fogging 3-FG [INHS]. Other material: 1 우, same data as paratypes.

Note. The three available male specimens all appear to have the tip of the aedeagus broken; thus the true shape of this structure remains unknown. This species is named in honor of Dr. Gustavo Moya-Raygoza, Universidad de Guadalajara, author of numerous studies on the ecology of leafhopper vectors, who facilitated the collection of this and many other undescribed species of Cicadellidae during the senior author's 2001 fieldwork in Mexico.

\section{Nelionidia pallescens, new species}

(Figs. 3-M; 4-L; 7-H-K)

Type locality: ca. La Huerta, Jalisco, Mexico. Diagnosis. Length of male $2.7 \mathrm{~mm}$, female 2.5 . Coloration pale yellow overall, apex of head infused with orange, paired brown spots on crown small. Male abdominal $2 \mathrm{~S}$ apodemes short, extended dorsomesad, not reaching posterior margin of sternite III. Pygofer (Fig. 4-L) with posteromedial emargination shallow, V-shaped; dorsal appendage extended ventrad, then abruptly bent posterad, distal section nearly straight, approximately equal to basal section in length. Aedeagus (Figs. 7-H, I) with apex blunt in lateral view, obtusangulate in posterior view, lateral processes elongate, extended ventrolaterad, weakly arcuate. Style apex (Fig. 7-J) resembling that of $N$. elliptica, but distance between first and second points shorter.

Material examined. Holotype $\sigma^{\top}$, MEXICO: Jalisco, ca. La Huerta, 480m, 19²7’47’N 
104³9'13”W, 16 Oct 2001, S.H.McKamey, et al., fogging 3-BY [INHS]; 1 ㄲ and 2 우 우 paratypes, same data [INHS].

Note. The species name refers to the pale overall coloration.

\section{Nelionidia pueblensis, new species}

(Figs. 3-L; 4-M; 7-L-O; 11-D, S)

Type locality: Nicolás Bravo, Puebla, Mexico. Diagnosis. Length of male $3.2-3.3 \mathrm{~mm}$, female $3.2 \mathrm{~mm}$. Coloration as in N. elliptica, but darkly pigmented areas more extensive and pale areas smaller. Male abdominal $2 \mathrm{~S}$ apodemes short, extended dorsomesad, not reaching posterior margin of sternite III (Fig. 11-D). Pygofer (Figs. 4-M, 11-S) with posteromedial emargination $\mathrm{V}$-shaped, extended to near base of segment; dorsal appendage broad basally, gradually tapered distally, with slender spine arising on mesal surface preapically and extended ventromesad. Aedeagus (Figs. 7-L, M) with shaft relatively broad in lateral view; apex in lateral view bearing acute anteriorly directed spine, apex in posterior view flattened and broad with long paired processes extended ventrad and sinuately curved. Style apex (Fig. 7-O) footlike, third point reduced to obtuse projection.

Material examined: Holotype $\sigma^{7}$, MEXICO: \#05-239 Puebla, Nicolás Bravo, 2600m, N18.6198 W97.3058, 10 Nov 2005, R. Rakitov [INHS]; 3 ठ ठ $\sigma^{7}$ paratype, MEXICO: \#05-230 Mexico, rt 190 10.8km W Rio Frio 3100m, N19.3195 W98.7436 9 Nov 2005, R. Rakitov [INHS]. Note. The species name refers to the state of Puebla, Mexico, in which the type series was collected.

\section{Neoimbecilla, new genus}

$$
\text { (Figs. 2-K, L; 3-J, Y; 4-N, O; 7-P-U) }
$$

Type species: Erythroneura kiperi Beamer, here designated.

Diagnosis. Length $3.1-3.6 \mathrm{~mm}$. Coloration (Figs. 2-K, L) pale yellow overall with few brown spots or more extensive brown markings; crown unmarked; mesonotum brown anterolaterally; scutellum black; forewing with indistinct brown markings in basal half.
Head subequal to pronotum in width; crown angulately produced, slightly longer medially than next to eye; coronal suture incomplete. Forewing (Fig. 3-J) inner apical cell oblique, $\mathrm{CuA}$ subequal in length to $\mathrm{CuP}$; third apical cell broadened distally; outer apical cell ca. $2 \mathrm{X}$ longer than wide. Hindwing vein RA obsolete; $\mathrm{m}$-cu present or MP confluent with $\mathrm{CuA}$ for short distance. Pygofer (Figs. 4-N, O) broadly emarginate to base dorsally; without oblique dorsolateral internal ridge; with conspicuous microtrichia distally; with few or no fine setae; basolateral setal group well differentiated, setae short and stout; apical lobe with group of short slender setae on mesal surface; dorsal appendage well developed, short, broad, movably articulated to pygofer margin dorsally, anteroventral lobe connected by well-sclerotized tendon to dorsal apodeme of aedeagus; ventral appendage absent; unsclerotized areas restricted to margin. First segment of anal tube basal arm weakly sclerotized, extended to junction of aedeagal tendon and pygofer appendage; microtrichia inconspicuous. Subgenital plate (Figs. 4-N, O) with basolateral angle obtuse; dorsal fold indistinct; submarginal row with 3-4 macrosetae; marginal row with short slender setae, continuous from basolateral angle to apex; distal lobe weakly to strongly expanded. Connective (Figs. 7-R, U) V-shaped; stem short, bifid, compressed. Style (Figs. 7-Q, T) preapical lobe prominent; apophysis smooth, expanded distally with extremely elongate mesally curved third point. Aedeagus (Figs. 7-P, S) with or without preatrium; dorsal apodeme broadly expanded in lateral view; shaft simple, tubular; processes present or absent; gonopore terminal.

Distribution. Southwestern USA and Mexico. Notes. The two included species were consistently paraphyletic with respect to the clade comprising Illinigina and Hymetta in the phylogenetic analysis, but constraining Neoimbecilla as monophyletic yielded trees only a single step longer. Despite the considerable differences between the two included species in coloration and pygofer structure, the short, stout, basolateral pygofer setae and the unique style apex seem sufficiently distinctive to warrant placing these species together in a genus distinct from other New World Erythroneurini. The genus name, which is feminine, was chosen to draw attention to the superficial similarity of the elongated style apex Neoimbecilla to those 
found among species of the Afrotropical genus Imbecilla Dworakowska. The type species was previously placed in the "ritana group" of Zygina by Young (1952).

\section{Included species:}

Neoimbecilla kiperi (Beamer, 1929b:124) (Erythroneura), new combination

Neoimbecilla latiplata, n. sp.

\section{Key to Males of Neoimbecilla}

1 Pygofer dorsal appendage with two or three prominent spines distally (Fig. 4-N); subgenital plate not expanded distally; aedeagus with pair of short distal processes (Fig. 7-S)

kiperi (Beamer)

1 ' Pygofer dorsal appendage tapered with apex truncate (Fig. 4-O), without spines; subgenital plate strongly broadened distally; aedeagus without distal processes latiplata, n. sp.

\section{Neoimbecilla latiplata, new species}

Type locality: Iguala, Guerrero, Mexico. Diagnosis. Coloration (Fig. 2-L) pale yellow overall; pronotum with pair of dark brown lateral maculae, mesonotum brown; scutellum black; forewing clavus with symmetrical diffuse brown infuscation in basal two-thirds, inner and middle anteapical cell infuscate distally. Male pygofer (Fig. 4-O) with dorsal appendage short, broad, tapered distally to truncate apex, curved downward. Subgenital plate (Fig. 4-O) with basolateral angle weakly developed, distal lobe strongly expanded, compressed, truncate apically. Aedeagus (Fig. 7-P) with shaft short, tapered in lateral view, apex depressed, without processes. Style (Fig. 7-Q) with third point nearly as long as rest of style, apex nearly reaching midline.

Material examined. Holotype $\diamond$, MEXICO: Guerrero, Iguala, 25-X-1941, DeLong, Good, Caldwell, and Plummer [INHS].

Note. The species name refers to the broad subgenital plate.

\section{Neozygina, new genus}

(Figs. 1-G; 2-M; 3-N, AA; 7-V-X; 11-C, P)

\section{Type species: Erythroneura ceonothana}

Beamer, here designated.

Diagnosis. Length $2.5-3.3 \mathrm{~mm}$. Coloration (Fig. 2-M) usually yellowish or reddish overall with brown infuscation, crown with pair of well-delimited preapical brown spots, ventral half of anteclypeus and lateral margins of clyp- eus usually brown, forewing variably infused with fuscous but without distinct color pattern, mesonotal triangles usually dark brown. Head slightly wider than pronotum; crown short, anterior and posterior margins subparallel, coronal suture incomplete. Forewing (Fig. 3-N) inner apical cell base oblique, length of $\mathrm{CuA}$ subequal to that of $\mathrm{CuP}$; third apical cell slender, parallel sided, outer apical cell approximately $2 \mathrm{X}$ longer than wide; anal vein poorly delimited. Hind wing (Fig. 3-AA) vein RA present; $\mathrm{m}$-cu crossvein absent, $\mathrm{CuA}$ confluent with MP for short distance or touching at one point. Sternite IX with median longitudinal internal ridge anteriorly. Pygofer (Figs. 5-B, 11-P) broadly emarginate to near base, without dorsolateral oblique internal ridge; surface usually with conspicuous microtrichia, with few or no fine setae; basolateral setal group inconspicuous or absent; apical lobe usually with distinct group of short slender setae on mesal surface; one or more macrosetae present near base of dorsal appendage; dorsal appendage rigidly attached, highly variable in form, arising from sclerotized dorsal ridge; posteroventral appendage present, usually elongate and curved dorsomesad; unsclerotized areas present distally along ventral margin and usually also between dorsal and ventral appendages. First segment of anal tube with basolateral arm well developed and strongly sclerotized, not closely associated with base of sclerotized ridge of pygofer; microtrichia conspicuous. Subgenital plate (Fig. 5-B) with basolateral angle acute; without conspicuous dorsal fold; with submarginal row of three macrosetae; marginal row 
of microsetae irregular but continuous from subbasal angle to near apex, setae short and slender; distal lobe digitiform, not expanded. Connective Y- or U-shaped; stem short, bifid, usually with median keel dorsally. Style (Fig. $7-W)$ preapical lobe greatly enlarged, usually truncate; apophysis, smooth, broad and truncate apically. Aedeagus (Fig. 7-V) with preatrium absent or short; dorsal apodeme narrow, weakly compressed, dorsal end expanded laterad, T-shaped in posterior view (Fig. 1-G), connections to pygofer membranous; shaft elongate, with paired apical or basal processes, or both; gonopore terminal or subterminal on posterior surface.

Distribution. Southern USA to Chile and Argentina.

\section{Included species:}

Neozygina abluta (McAtee, 1924d:132) (Erythroneura), new combination Neozygina apacha (Baker, 1925b:537) (Erythroneura), new combination Neozygina arida (Beamer, 1937b:31) (Erythroneura), new combination Neozygina balli (Beamer, 1932i:125) (Erythroneura), new combination Neozygina bimaculata (Baker, 1903d:9) (Typhlocyba), new combination Neozygina ceonothana (Beamer, 1934c:287) (Erythroneura), new combination Neozygina davisi (Beamer, 1934d:96) (Erythroneura), new combination Neozygina huachucana (Beamer, 1934c:287) (Erythroneura), new combination Neozygina penapacha (Beamer, 1941a:18) (Erythroneura), new combination Neozygina quadricornis (Beamer, 1930b:431) (Erythroneura), new combination

\section{Rossmoneura, new genus}

$$
\text { (Figs. 2-N; 5-A; 7-Y, Z) }
$$

Type species: Erythroneura tecta McAtee, here designated

Diagnosis. Length 2.5-3.6 mm. Ground color pale yellow, dorsum heavily marked with dark brown, tinged with red; crown usually with pair of somewhat diffuse brown spots preapically (Fig. 2-M); face with anteclypeus and lateral margin of frontoclypeus near antennal pit dark brown; mesosternum and mesonotal triangles dark brown; forewing with large transcommisural pale area near base, another covering distal part of clavus and extended to base of inner apical cell. Head subequal to or narrower than pronotum, crown short, slightly longer mesad than next to eye. Wing venation as in Erasmoneura. Male genital capsule and genitalia as in Erasmoneura except as follows: pygofer (Fig. 5-A) without distinct microtrichia distally, dorsal appendage without distinct basal line of
Notes. The genus name, which is feminine, combines "neo," signifying new, with "Zygina," the name of the genus in which the included species were formerly placed, and is meant to signify that the genus is endemic to the New World. The monophyly of Neozygina is supported by the presence of one or more appendage, a feature unique among New World Erythroneurini, and the absence of a ventrolateral setal group on the pygofer. All of the North American species previously assigned to the "ceonothana group" of Zygina (sensu lato) by Young (1952) belong to this genus. Many undescribed species of this genus occur in Mexico, Central and South America. 
Prof. Herbert H. Ross, the pioneering insect systematist and educator whose well-curated collections largely form the basis for this revi- sion, with the last three syllables of Erasmoneura, the genus-group taxon in which the three included species were most recently placed.

\section{Included species:}

Rossmoneura calva (Beamer, 1946b:22) (Erythroneura), new combination Rossmoneura carbonata (McAtee, 1920a:289) (Erythroneura), new combination Rossmoneura tecta (McAtee, 1920a:288) (Erythroneura), new combination

\section{Zyginama, new genus}

(Figs. 1-E; 2-O; 3-O; 7-AA-CC)

\section{Type species: Erythroneura ritana Beamer,} here designated.

Diagnosis. Length $2.5-3.5 \mathrm{~mm}$. Coloration (Fig. 2-O) pale yellow to brownish overall, crown often with pair of somewhat diffuse brown or reddish preapical spots, pronotum and forewing often with symmetrical reddish markings. Head slightly narrower than pronotum, anterior margin angulately produced, crown longer medially than next to eyes; coronal suture complete. Wing venation (Figs. 3-O, BB) as in Neozygina. Sternite IX with median longitudinal internal ridge anteriorly. Pygofer (Fig. 1-E) broadly emarginate to near base, without dorsolateral oblique ridges; with indistinct microtrichia distally; fine setae very sparse or absent; ventrolateral setal group well developed, usually consisting of several macrosetae; distal lobe with several short slender setae on mesal surface; without macrosetae near base of dorsal appendage; dorsal appendage simple, spinelike, rigidly attached, arising from sclerotized dorsal ridge; ventral appendage present usually, elongate and curved dorsomesad (very short and inconspicuous in some spp.); unsclerotized areas present distally along ventral margin and usually also between dorsal and ventral appendages. First segment of anal tube with basolateral arm well developed and strongly sclerotized, not closely associated with base of sclerotized ridge of pygofer; distal ring with conspicuous microtrichia. Subgenital plate (Fig. 1-E) with basolateral angle acute; without conspicuous dorsal fold; with submarginal row of three macrosetae; marginal row of microsetae irregular but more or less continuous from subbasal angle to near apex, setae short and slender; distal lobe expanded in lateral view. Connective (Fig. 7-CC) U-or V-shaped; stem short, bifid, usually with median keel dorsally. Style (Fig. 7-BB) preapical lobe greatly enlarged, usually truncate; apophysis smooth, broad and truncate apically. Aedeagus (Fig. 7-AA) with preatrium short; dorsal apodeme well developed, weakly compressed, expanded dorsally in lateral view, dorsal end expanded laterad, T-shaped in posterior view, connections to pygofer membranous; shaft highly variable interspecifically, often with paired apical and/or basal processes; gonopore terminal or subterminal on posterior surface.

Distribution. Southern USA to Peru.

Note. The name is feminine and is modified from that of the genus in which the included species were previously placed. Most of the species formerly assigned to the "ritana group" of Zygina (s.l.) by Young (1952) apparently belong in this genus. Numerous undescribed species of this genus occur in Mexico and in Central and South America.

\section{Included species:}

Zyginama agnata (Knull \& Auten 1938a:537) (Erythroneura), new combination Zyginama ales (Beamer 1932I: 124) (Erythroneura), new combination Zyginama arizonica (Knull \& Auten 1938a:536) (Erythroneura), new combination Zyginama aucta (McAtee 1920a:279) (Erythroneura), new combination Zyginama bilocularis (Van Duzee 1924a:233) (Erythroneura), new combination Zyginama blanda (Knull \& Auten 1938a:538) (Erythroneura), new combination Zyginama canyonensis (Beamer 1929b:120) (Erythroneura), new combination Zyginama casta (Beamer 1929b:118) (Erythroneura), new combination Zyginama cimarroni (Beamer 1929b:119) (Erythroneura), new combination Zyginama cornigera (Beamer 1937b:31) (Erythroneura), new combination Zyginama dentata (Gillette 1898a:765) (Erythroneura), new combination 
Zyginama erosa (McAtee 1924c:36) (Erythroneura), new combination Zyginama grandis (Beamer 1929b:127) (Erythroneura), new combination Zyginama inclita (Beamer 1934a:44) (Erythroneura), new combination Zyginama iguala (Ross 1965:263) (Erythroneura), new combination Zyginama inornata (McAtee 1924d:132) (Erythroneura), new combination Zyginama merita (Beamer 1932I:127) (Erythroneura), new combination Zyginama modula (Knull \& Auten 1938a:535 ) (Erythroneura), new combination Zyginama munda (Knull \& Auten 1938a:534) (Erythroneura), new combination Zyginama nicholi (Beamer 1927a:30) (Erythroneura), new combination Zyginama novella (Knull \& Auten 1938a:536) (Erythroneura), new combination Zyginama nuda (Knull \& Auten 1938a:538) (Erythroneura), new combination Zyginama obscura (Beamer 1929b:117) (Erythroneura), new combination Zyginama pallenta (Beamer 1929b:117) (Erythroneura), new combination Zyginama pinalensis (Beamer 1929b:119) (Erythroneura), new combination Zyginama ritana (Beamer 1934c:286) (Erythroneura), new combination Zyginama rubicunda (Beamer 1929b:126) (Erythroneura), new combination Zyginama sola (Knull \& Auten 1938a:534) (Erythroneura), new combination Zyginama spectabilis (Knull \& Auten 1938a:533) (Erythroneura), new combination Zyginama ternaria (Van Duzee 1924a:235) (Erythroneura), new combination Zyginama triceroprocta (Beamer 1929b:118) (Erythroneura), new combination Zyginama tricolor (Beamer 1929b:124) (Erythroneura), new combination Zyginama tripunctata (Beamer 1929b:124) (Erythroneura), new combination Zyginama unicolor (Beamer 1929b:120) (Erythroneura), new combination Zyginama utahna (Beamer 1937b:32) (Erythroneura), new combination

\section{South American Genera}

\section{Amazygina, new genus}

(Figs. 1-I; 3-P, CC; 5-C-F; 8-A-P; 11-E-H, T)

Type species: A. decaspina, n. sp., here designated.

Diagnosis. Length $2.9-3.3 \mathrm{~mm}$. Coloration pale yellow or white overall; crown without paired spots, with or without dark median anterior macula (as in Fig. 2-P). Head wider than pronotum; crown short with anterior and posterior margins subparallel, without paired spots, some species with dark median apical macula, coronal suture incomplete. Forewing (Fig. 3-P) relatively broad, inner apical cell base oblique, length of $\mathrm{CuA}$ subequal to that $\mathrm{CuP}$; third apical cell broadened distally, outer apical cell approximately $2 \mathrm{X}$ longer than wide; anal vein relatively well delimited. Hindwing (Fig. 3-CC) vein RA present; $\mathrm{m}$-cu crossvein absent, $\mathrm{CuA}$ confluent with MP for short distance. Sternite IX with distinct median internal ridge. Pygofer (Figs. 5-C-F, 11-T) broadly emarginate dorsally to or near base of segment, without dorsolateral oblique ridges; dorsal appendage rigidly attached, arising near apex from sclerotized dorsal ridge, usually with one or more teeth; surface smooth, without distinct microtrichia, with a few long fine setae; basolateral setal group well developed with several large setae; dorsoapical lobe with distinct group of short slender setae mesad of appendage. First segment of anal tube (Fig. 11-T) with basolateral arms well developed and strongly sclerotized, articulated to base of sclerotized ridge of pygofer; microtrichia consipcuous. Subgenital plate with basolateral angle usually acute; dorsal fold moderately well developed; submarginal row with 3-4 macrosetae; marginal row of microsetae irregular, very sparse distally, setae short and slender; distal lobe somewhat compressed and broadened. Connective (Fig. 8-C, G) Y or U-shaped; stem, when present, short, bifid. Style (Fig. 8-D) preapical lobe prominent, broadly rounded; apophysis slightly curved, apex truncate with imbricate texture at least ventrally. Aedeagus (Figs. 8-A, E, I, M) highly variable interspecifically, dorsal apodeme well developed, expanded and T-shaped in posterior view, dorsal connections to pygofer membranous; with long paired processes arising near base; with or without unpaired dorsal process anterad of gonopore-bearing shaft; gonopore terminal or subterminal on posterior surface. 
Distribution. Ecuador.

Note. The genus name, which is feminine, was formed by combining "Amazon" the region in which the genus occurs, with "Zygina", the genus to which species run in Young's (1952) key. This genus appears to be endemic to South America. No previously described North American species belong to this genus, nor are any of the undescribed North American species presently in hand assignable to Amazygina.
The genus is described based on four new species, all of which were found in rainforest canopy-fogging samples from eastern Ecuador. Some specimens of all four species were collected in the same sample, suggesting that they were feeding on the same (unknown) host plant. Although the samples contained males and females, reliable morphological features separating females were not found. Males are separable using the following key.

\section{Included species:}

Amazygina chela, n. sp.

Amazygina compressa, $\mathrm{n}$. sp.

Amazygina decaspina, n. sp.

Amazygina depressa, $\mathrm{n}$. sp.

\section{Key to Males of Amazygina}

1 Pygofer with dorsal appendage reduced to short, toothed projection (Figs. 5-D, F)

1 ' Pygofer with dorsal appendage well developed, spinelike (Figs. 5-C, E)

2 Aedeagus without unpaired dorsal process between gonopore-bearing shaft and base; shaft with two pairs of long processes arising from base and extended along shaft and pair of slender retrorse spines arising just basad of gonopore (Fig. 8-E, F) ...............................compressa, n. sp. 2' Aedeagal with unpaired dorsal process between gonopore-bearing shaft and base; with two pairs of basal processes arising near base, anterior pair trifircate; without retrorse spines just basad of gonopore (Fig. 8-M, N) decaspina, n. sp.

3 Aedeagus with large, depressed, dorsal process between base and gonopore-bearing shaft, gonopore-bearing shaft tubular, with pair of slender apical spines (Fig. 8-I, J) ............depressa, n. sp. 3 ' Aedeagus in lateral view with a single short, tapered shaft bearing pair of slender elongate dorsally directed spines (Fig. 8-A, B) chela, n. sp.

\section{Amazygina chela, new species}

(Figs. 5-C; 8-A-D; 11-E)

Diagnosis. Length of male $3 \mathrm{~mm}$. Male $2 \mathrm{~S}$ apodemes short, narrow, extended dorsomesad (Fig. 11-E). Crown unmarked; anteromedial area and lateral triangles of mesonotum brown. Pygofer dorsal appendage C-shaped, with dorsal preapical tooth (Fig. 5-C). Aedeagus (Figs. 8-A, B) with short tapered gonopore-bearing shaft and pair of elongate slender processes arising near base of shaft and extended posterodorsad. Style apex footlike, second point long (Fig. 8-D).

Material examined. Holotype $\diamond$, ECUADOR: Orellana, Transect Ent. 1 km S Onkonegare Camp, Reserva Etnica Waorani,
00³9'10"S, 076²6'00"W; lot\#1440, 7-Feb96, T. L. Erwin, et al., fogging terra firme forest [USNM]; $2 \sigma^{7} \sigma^{7}$ paratypes, same data except lot\#1406, 4-Feb-96 [USNM], and lot\#1028, 12-Feb-99 [INHS].

Note. The species name refers to the clawlike pygofer appendage. The size of the dorsal preapical tooth of this appendage varies intraspecifically, with some specimens having the spine larger than shown in Fig. 5-C.

\section{Amazygina compressa, new species}

(Figs. 2-P; 5-D; 8-D-F; 11-F)

Diagnosis. Length of male $3.3 \mathrm{~mm}$. Crown apex with diffuse median brown macula (Fig. 2-P); mesonotum brown anteromedially; lateral triangles brown. Male $2 \mathrm{~S}$ apodemes short, 
rounded, extended dorsomesad (Fig. 11-F). Pygofer dorsal appendage short, bidentate (Fig. 5-D). Aedeagal shaft (Figs. 8-E, F) not divided, tubular, apex compressed, obliquely truncate in lateral view; pair of slender retrorse spines just basad of gonopore on posterior preapical surface; paired basal processes somewhat flattened and twisted preapically, as long as shaft. Style (Fig. 8-H) with preapical lobe present but not prominent; apex with two points subequal in size.

Material examined. Holotype $\sigma^{\nearrow}$, ECUADOR: Orellana, Transect Ent. $1 \mathrm{~km} \mathrm{~S}$ Onkonegare Camp, Reserva Etnica Waorani, 00³9'10'S, 076²6'00'W; lot\#1440, 7-Feb96, T. L. Erwin, et al., fogging terra firme forest [USNM]. Additional material: 1 우, same data as holotype [USNM].

Note. The species name refers to the compressed distal portion of the aedeagal shaft.

\section{Amazygina decaspina, new species}

(Figs. 1-I; 3-P, CC; 5-F; 8-M-P; 11-H)

Diagnosis. Length of male $3 \mathrm{~mm}$. Crown apex with diffuse median brown macula (as in Fig. 2-P); mesonotum brown anteromedially, lateral triangles brown; mesosternum brown. Male 2S apodemes robust, well separated at base, rounded, extended posteromesad, nearly reaching posterior margin of sternite III (Fig. 11-H). Pygofer dorsal appendage (Fig. 5-F) similar to that of A. compressa. Aedeagus (Figs. 8-M, N) with dorsal process between base and gonopore-bearing shaft compressed, acuminate in lateral view; gonopore-bearing shaft tubular with apex acuminate; two pairs of basal processes present; posterior pair slender and straight, extended dorsad and curved posterad apically; anterior pair divided near midlength into three processes, one extended anterad, one short slender pair extended dorsad, and a longer pair extended dorsad and strongly arched anterad beyond shaft apex. Style apex hatchet-like (Fig. 8-P).

Material examined. Holotype $\sigma^{\top}$, ECUADOR: Orellana, Transect Ent. $1 \mathrm{~km} \mathrm{~S}$ Onkonegare Camp, Reserva Etnica Waorani, 00³9'10”S, 076²6'00”W; lot\#1560, 21-Jun96, T. L. Erwin, et al., fogging terra firme forest [USNM]. Paratypes: 4 ठ ठ holotype; 6 ठ Љ , same data except lot\#1440, 7-Feb-96 [USNM, INHS]; 1 ○', ECUADOR:
Orellana, Tiputini Biodiversity Sta. nr Yasuni Nat. k 220-250m, 00³7'55'S, 07608'39”'W, 7-Feb-99, T. L. Erwin, et al., Lot\#2040, Transect $\mathrm{T} / 5$, fogging terra firme forest [USNM]. Additional material: 2 우 우, same data as holotype; 2 우 우, same data except lot\#1440, 7-Feb-96 [USNM, INHS].

Note. The species name refers to the aedeagus, which has a total of 10 spinelike processes, including the gonopore-bearing shaft.

\section{Amazygina depressa, new species}

$$
\text { (Figs. 5-E; 8-I-L; 11-G, T) }
$$

Diagnosis. Length of male $3 \mathrm{~mm}$. Male $2 \mathrm{~S}$ apodemes subquadrate, not reaching posterior margin of sternite III (Fig. 11-G). Color uniformly pale yellow except forewing apex weakly infuscated. Pygofer dorsal appendage long and slender, strongly arcuate, with short preapical spine extended posterad (Fig. 5-E). Aedeagus (Figs. 8-I, J) with large strongly depressed anterior dorsally directed process; process in posterior view broad, tapered and acute apically; gonopore-bearing shaft short, slender, tubular; apex truncate, with pair of short, slender posterolateral spines; basal processes as long as anterior process. Style apex small, footlike (Fig. 8-L).

Material examined. Holotype $\sigma^{\top}, \mathrm{EC}-$ UADOR: Orellana, Transect Ent. $1 \mathrm{~km} \mathrm{~S}$ Onkonegare Camp, Reserva Etnica Waorani, 00³9'10"S, 076 $26^{\prime} 00^{\prime \prime} \mathrm{W}$; lot\#1560, 20-June96, T. L. Erwin, et al., fogging terra firme forest [USNM]; $1 \sigma^{7}$ paratype, same data [INHS]; 1 male paratype, same data except lot\#1440, 7-Feb-96 [USNM]. Additional material: 3 우 우, same data as holotype; 1 우, same data as second male paratype [USNM, INHS].

Note. The species name refers to the large depressed dorsal process of the aedeagus.

\section{Hamagina, new genus}

$$
\text { (Figs. 2-P; 3-Q; 5-G-I; 9-A-L; 11-I-K, U) }
$$

Type species: $H$. spinigera, n. sp., here designated.

Diagnosis. Coloration (Fig. 2-P) pale yellow overall, dorsum heavily infuscated; crown with large median anterior brown macula; anterior half and lateral triangles of mesonotum, and mesosternum dark brown; forewing apex 
infuscated. Wing structure and venation as in Amazygina. Sternite IX with median longitudinal internal ridge anteriorly. Pygofer (Figs. 5-G-I) shallowly emarginate dorsally (Fig. 11-U); without oblique anterolateral ridge; surface smooth, without conspicuous microtrichia or fine setae; basolateral setal group well differentiated, setae somewhat enlarged; distal lobe with well-developed preapical and apical group of short fine setae; dorsal margin without macrosetae; dorsal appendage well developed, short, falcate, rigidly attached to pygofer margin; ventral appendage present, slender, curved dorsomesad; lobe between appendages wealky sclerotized. First segment of anal tube (Fig. 11-U) with basolateral arm well developed, articulated near base of dorsal appendage; microtrichia conspicuous. Subgenital plate (Figs. 5-G-I) with basolateral angle prominent; dorsal fold weakly developed; submarginal row with three macrosetae; marginal row with short fine setae, more sparsely distributed distally; distal lobe not expanded, with or without prominent dorsal spine. Connective (Figs. 9-B, E, H) Y- or U-shaped, stem short, bifid, overlapping aedeagal base. Style (Fig. 9-C) with preapical lobe prominent; apophysis smooth, apex attenuate or with two points. Aedeagus (Fig. 9-A) with preatrium short; dorsal apodeme crescent-shaped in lateral view, T-shaped in dorsal view, connections to pygofer membranous; with large median dorsal process between base and gonopore-bearing shaft; shaft with paired basal or preapical spines or processes; gonopore terminal.

Distribution. Ecuador and Peru.

Notes. The genus name, which is feminine, is derived in part from the Latin word "hamus" (hook) and refers to the large distal spine on the subgenital plate of two of the three known species. Phylogenetic analysis recovered the genus as sister to a clade comprising Amazygina and Perugina, $\mathrm{n}$. gen. The genus is described based on three new species, two from lowland Amazonian rainforest and one from a cloud forest in the eastern Andean foothills.

\section{Included species:}

Hamagina pascoensis, n. sp.

Hamagina serrata, $\mathrm{n}$. sp.

Hamagina spinigera, $\mathrm{n}$. sp.

\section{Key to Males of Hamagina}

1 Subgenital plate with large distal spine (Figs. 5-H, I)

1'Subgenital plate without distal spine (Fig. 5-G)

pascoensis, n. sp.

2 Subgenital plate spine extended dorsad, gonopore-bearing shaft of aedeagus with numerous small lateral spines (Fig. 9-D) serrata, n. sp. 2' Subgenital plate spine extended mesad (Fig. 11-U), gonopore-bearing shaft of aedeagus without lateral spines (Fig. 9-G) spinigera, n. sp.

\section{Hamagina pascoensis, new species}

(Figs. 2-Q; 5-G; 9-A-C; 11-I)

Diagnosis. Length of male $3.7 \mathrm{~mm}$. Male $2 \mathrm{~S}$ apodemes broad, triangular, extended nearly to posterior margin of sternite III (Fig. 11I). Pygofer (Fig. 5-G) dorsal appendage with base perpendicular to sclerotized ridge, apex weakly arcuate, expanded and triangular in dorsal view; ventral appendage short, slender, curved posterodorsad along pygofer margin. Subgenital plate (Fig. 5-G) without distal spine.
Aedeagus (Fig. 9-A) with gonopore-bearing shaft with pair of small ventral preapical spines and pair of apical triangular teeth; pair of elongate slender processes arising near base and extended dorsad of shaft well beyond apex. Style apex (Fig. 9-C) footlike, heel (first point) prominent, toe (second point) elongate.

Material examined. Holotype $\sigma^{\top}$, PERU: Pasco, Yanachaga-Chemillen N.P., 10³2'39.7'S, $75^{\circ} 22^{\prime} 0.1^{\prime \prime} \mathrm{W}, 2300 \mathrm{~m}, 10-13$ Oct 2002, D.

Takiya, C. Peña, R. Rakitov, Malaise trap acr. R. San Alberto [USML].

Note. The species name refers to the Peruvian department where the type specimen was collected. 


\section{Hamagina serrata, new species}

(Figs. 5-H; 9-D-F; 11-J)

Diagnosis. Length of male $3.2 \mathrm{~mm}$. Male $2 \mathrm{~S}$ apodemes broad, triangular, extended nearly to posterior margin of sternite III (Fig. 11J). Pygofer (Fig. 5-H) with dorsal appendage short, bifid distally, elevated above distal lobe; ventral appendage very short, slender, extended dorsad. Subgenital plate (Fig. 5-H) with prominent dorsally directed distal spine. Aedeagus (Fig. 9-D) with gonopore-bearing shaft tubular, with serrate flange posterolaterally and pair of posteroapical spines. Style apex (Fig. 9-F) with heel (point 1) poorly developed, section distad of heel elongate and sinuate.

Material examined. Holotype $\sigma^{\top}$, PERU: Madre de Dios, Rio Tambopata Res. $30 \mathrm{~km}$ (air) SW Pto. Maldonado, 290 m. 1250'S, $069^{\circ} 17^{\prime} \mathrm{W}$, Smithsonian Institution Canopy Fogging Project, T. L. Erwin, et al. colls., 14 Sep 1984 01/02/46 [USNM].

Note. The species name refers to the serrate ventrolateral margin of the gonopore-bearing shaft of the aedeagus.

\section{Hamagina spinigera, new species}

$$
\text { (Figs. 3-Q; 5-I; 9-G-I; 11-K, U) }
$$

Diagnosis. Length of male $2.8-3 \mathrm{~mm}$. Male $2 \mathrm{~S}$ apodemes short, extended dorsomesad (Fig. 11-K). Pygofer (Fig. 5-I) with dorsal appendage broad, short, curved posteroventrad; ventral spine slender, curved dorsad. Subgenital plate with prominent preapical spine extended mesad (Figs. 5-I, 11-U). Aedeagus (Fig. 9-G) with gonopore-bearing shaft tridentate apically; pair of long, slender, nearly straight processes extended posterodorsad between basal spine and gonopore bearing shaft. Style apex (Fig. 9I) flukelike, points subequal in size.

Material examined. Holotype $\sigma^{\top}$, ECUADOR: Orellana, Transect Ent. $1 \mathrm{~km} \mathrm{~S}$ Onkonegare Camp, Reserva Etnica Waorani, 00³9'10'S, $076^{\circ} 26^{\prime} 00^{\prime \prime} \mathrm{W}$; lot\#1684, 1-Oct1996, T. L. Erwin, et al., fogging terra firme forest [USNM]; 3 ठ ठ lot\#1573, 22-Jun-1996; lot\#1084, 2-Jul-95; lot\#1211, 6-Oct-95 [USNM, INHS].

Note. The species name refers to the well-developed distal spine on the subgenital plate.

\section{Napogina, new genus}

(Figs. 2-R; 5-J, K; 10-A-H; 11-L)

Type species: N. sinuata, n. sp., here designated

Diagnosis. Length $2.8 \mathrm{~mm}$. Closely resembling Amazygina overall, but differing as follows: pygofer appendage simple, tapered, not elevated above dorsal margin, without teeth or basal process (Figs. 5-J, K); style apophysis (Figs. 10-C, H) attenuate, without distinct sculpturing, preapical heel small or absent; aedeagus (Figs. 10-A, B, E, F) with paired subapical processes, basal processes absent.

Distribution. Ecuador.

Notes. The name Napogina, which is feminine, is based on that of the Napo region of Ecuador where both included species occur. The phylogenetic analysis recovered the genus as a monophyletic sister to the clade comprising new genera Hamagina and Spinigina.

\section{Included species:}

Napogina recta, n. sp.

Napogina sinuata, n. sp.

\section{Key to Males of Napogina}

1 Aedeagus (Fig. 10-E) with shaft arched posterad distally, with single pair of lamelliform processes, each bearing three fingerlike branches; style apex footlike (Fig. 10-H) ...........sinuata, n. sp. 1 'Aedeagus (Fig. 10-A) with shaft apex extended anterodorsad, with two pairs of simple, spinelike processes; style apex slender, acuminate (Fig. 10-C) .recta, n. sp. 


\section{Napogina recta, new species}

(Figs. 5-J; 10-A-C; 11-L)

Diagnosis. Length of male $2.8 \mathrm{~mm}$. Crown unmarked; anteromedian area and lateral triangles of mesonotum dark brown. Male $2 \mathrm{~S}$ apodemes large, triangular, surpassing posterior margin of sternite III (Fig. 11-L). Pygofer with dorsal emargination extended nearly to base, broadly parabolic; dorsal appendage small, digitiform, arising near apex of dorsal margin but not extended to margin; apical margin truncate; ventrolateral setal group with 6-7 conspicuous setae. Subgenital plate (Fig. 5-J) with distal lobe broad, compressed, strongly bent dorsad. Connective (Fig. 10-D) compact, arms short and broad; stem bifid, strongly overlapping aedeagal preatrium. Style (Fig. 10-C) preapical lobe prominent, subangulate; apophysis slender, attenuate, evenly curved dorsolaterad. Aedeagus (Figs. 10-A, B) with preatrium short; dorsal apodeme tapered in lateral view, T-shaped in posterior view; shaft massive, long, recurved subrectangularly, with irregular serrate posterolateral flanges and two pairs of slender preapical processes, both extended anteroventrad, penultimate pair longer and curved more strongly anterad; gonopore apical.

Material examined. Holotype $\sigma^{\top}, \mathrm{EC}-$ UADOR: Orellana, Transect Ent. $1 \mathrm{~km} \mathrm{~S}$ Onkonegare Camp, Reserva Etnica Waorani, 00³9'10'S, 076²6'00”'W; lot\#1494, 10-Feb1996, T. L. Erwin, et al., fogging terra firme forest [USNM]; 1 万ग paratype, same data except lot\#959. 9-Feb-99 [USNM]. Additional material: 2 우 우, same data as paratype [USNM].

Note. The species name refers to the relatively straight distal section of the aedeagal shaft.

\section{Napogina sinuata, new species}

$$
\text { (Figs. 2-R; 5-K; 10-E-H) }
$$

Diagnosis. Length of male $2.8 \mathrm{~mm}$. Crown (Fig. 2-R) with indistinct pair of brown spots apically; anteromedial area and lateral triangles of mesonotum and mesosternum dark brown. Male 2S apodemes large, triangular, extended to posterior margin of sternite III. Pygofer (Fig. 5-K) with dorsal emargination extended to base, broad, nearly parallel-sided; dorsal appendage moderately large, falcate, curved ventromesad, not extended to apical margin; apical margin truncate, with poorly sclerotized semicircular area ventrad of dorsal appendage; ventrolateral setal group with 4 conspicuous setae. Subgenital plate (Fig. 5-K) with distal lobe curved dorsad, compressed, somewhat expanded distally. Connective broadly U-shaped; stem bifid, strongly overlapping aedeagal preatrium. Style (Fig. 10-H) with preapical lobe well developed but small; apophysis elongate with footlike apex, heel bent laterad. Aedeagus (Figs. 10-E, F.) with preatrium short; dorsal apodeme slightly expanded dorsally in lateral view, T-shaped in posterior view; shaft depressed and straplike, in lateral view bent rectangularly, with distal portion arched posterad toward apex; two pairs of preapical lateral processes present, penultimate pair slender and spinelike, more distal pair longer, flattened and bifurcate, with ventral branch twisted and overlapping dorsal branch; gonopore apical; median distal spine present dorsad of gonopore. Material examined. Holotype $\sigma^{\top}$, ECUADOR: Orellana, Transect Ent. $1 \mathrm{~km} \mathrm{~S}$ Onkonegare Camp, Reserva Etnica Waorani, 00³9'10"S, 076²6'00'W; lot\#1406, 4-Feb1996, T. L. Erwin, et al., fogging terra firme forest [USNM]; 2 శ $\sigma^{7}$ paratypes, same data except lot\#1579, 22-Jun-96 [USNM, INHS]. Note. The species name refers to the sinuate aedeagal shaft.

\section{Perugina, new genus}

$$
\text { (Figs. 3-R; 5-L; 10-I-L; 11-M) }
$$

Type species: $P$. denticula, n. sp., here designated.

Diagnosis. Length $2.8 \mathrm{~mm}$. Color pale yellow overall; crown with pair of indistinct brown spots preapically (as in Fig. 2-R); anteromedial area and lateral triangles of mesonotum and mesosternum dark brown. Head wider than pronotum; crown short with anterior and posterior margins subparallel, without distinct markings, coronal suture incomplete. Wing venation (Fig. 3-R) as in Amazygina. Sternite IX with distinct median and two lateral longitudinal internal ridges. Pygofer (Fig. 5-L) broadly emarginate to near base, with pair of short broad dorsolateral apodemes, without dorsolateral oblique ridges; dorsal appendage rigidly attached, small, dentate, arising near apex from sclerotized dorsal ridge; surface smooth, without distinct microtrichia, numerous long 
fine setae; basolateral setal group well developed with several large setae; dorsoapical lobe with distinct group of short slender setae mesad of appendage. First segment of anal tube with basolateral arm well developed, strongly sclerotized, articulated to base of sclerotized ridge of pygofer; microtrichia conspicuous. Subgenital plate (Fig. 5-L) with basolateral angle acute; with dorsal fold moderately well developed; submarginal row with three macrosetae; marginal row of microsetae irregular but continuous from subbasal angle to near apex, setae short and slender. Connective (Fig. 10-K) Y-shaped, stem short, bifid, overlapping aedeagal preatrium. Style (Fig. 10-L) preapical lobe prominent, broadly rounded; apophysis slightly curved, apex avicephaliform with imbricate texture. Aedeagus (Figs. 10-I, J) with preatrium short, articulated near fork of connective; dorsal apodeme long, narrow, compressed, dorsal connections to pygofer membranous; shaft elongate, tubular, with long paired basal processes, apex acuminate; gonopore terminal, surrounded by minute denticuli.

Distribution. Peru.

Notes. The genus name, which is feminine, is based on that of the country where specimens of the only known species were collected. Phylogenetic analysis placed Perugina as sister to Amazygina. This genus is readily identified by the structure of the pygofer, which lacks appendages and is conspicuously clothed with long, fine setae.

\section{Included species:}

Perugina denticula, n. sp.

\section{Perugina denticula, new species}

Diagnosis. Length of male $2.8 \mathrm{~mm}$. Coloration as described for genus. Male abdomen with $2 \mathrm{~S}$ apodemes short, rounded, extended dorsomesad (11-M). Aedeagus (Figs. 10-I, J) with basal processes long, sinuate. Style apex (Fig. 10-L) subtruncate with small median tooth.

Material examined. Holotype $\sigma^{\top}$, PERU: Madre de Dios, Rio Tambopata Res. $30 \mathrm{~km}$ (air) SW Pto. Maldonado, 290 m. 1250'S, $069^{\circ} 17^{\prime} \mathrm{W}$, Smithsonian Institution Canopy Fogging Project, T. L. Erwin, et al. colls., 06 Sep 1984 05/02/156 [USNM]; 3 ठ $\sigma^{\top}$ paratypes, same data [USNM, INHS]; 1 का paratype, same data except 07 May 1984, 05/02/072 [USNM].

\section{Spinigina, new genus}

\author{
(Figs. 3-S; 5-M, N; 10-M-T; 11-N)
}

Type species: $S$. hirsuta, n. sp., here designated.

Diagnosis. Length $3 \mathrm{~mm}$. Head wider than pronotum; crown short with anterior and posterior margins subparallel, with pair of somewhat diffuse brown spots preapically, coronal suture incomplete. Wing venation (Fig. 3-S) as in Amazygina. Sternite IX with distinct median longitudinal internal ridge. Pygofer (Figs. 5-M, N) broadly emarginate to near base, without dorsolateral oblique ridges; dorsal appendage rigidly attached, falcate, arising near apex from sclerotized dorsal ridge; surface smooth, without distinct microtrichia, with sparse fine setae; basolateral setal group well developed with several large setae; apical lobe with distinct group of short slender setae; ventral appendage short and inconspicuous or absent. First segment of anal tube with basolateral arm well developed and strongly sclerotized, articulated to base of sclerotized ridge of pygofer; pair of broad spinose ventolateral lobes distally. Subgenital plate (Figs. 5-M, M) with basolateral angle obtuse; without conspicuous dorsal fold; with submarginal row of three macrosetae; marginal row of microsetae irregular but continuous from subbasal angle to near apex, setae short and slender. Connective (Figs. 10-O, R) U- or V-shaped, stem short, bifid. Style (Figs. 10-P, Q) preapical lobe prominent, truncate; apophysis sickle-shaped, apex attenuate with small preapical tooth. Aedeagus (Figs. 10-M, $\mathrm{N}, \mathrm{S}, \mathrm{T}$ ) with preatrium short, articulated near fork of connective; dorsal apodeme long, narrow, and weakly compressed, dorsal connections to pygofer membranous; shaft elongate, depressed, with long paired distal processes; gonopore preapical or terminal.

\section{Distribution. Peru.}

Notes. This genus is readily distinguished by the reduced or absent ventral pygofer appendage, the long slender style apophysis, and the presence of numerous short spines on the first segment of the anal tube, a feature unique among known Erythroneurini and upon which the genus name (feminine) is based. The genus is based on two new species from Rio Tambopata Reserve, Madre de Dios, Peru, described below. 


\section{Included species:}

Spinigina hirsuta, n. sp.

Spinigina quadrispinosa, n. sp.

\section{Key to Males of Spinigina}

1 Pygofer without ventral appendage (Fig. 5-N); aedeagus (Figs. 10-S, T) with two pairs of distal processes, apex depressed, broad in posterior view; style apex (Fig. 10-Q) evenly curved laterad, without distinct heel-like angle quadrispinosa, $\mathrm{n} . \mathrm{sp}$. 1' Pygofer with small ventral appendage (Fig. 5M); aedeagus (Figs. 10-M, N) with one pair of distal processes, apex compressed, slender in posterior view; style apex (Fig. 10-P) abruptly bent laterad, with distinct heel-like angle hirsuta, n. sp.

\section{Spinigina hirsuta, new species}

(Figs. 3-S; 5-M; 10-M-P; 11-N)

Diagnosis. Length of male $3 \mathrm{~mm}$. Male $2 \mathrm{~S}$ apodemes large, rounded, extended beyond posterior margin of sternite III (Fig. 11-N). Pygofer (Fig. 5-M) with dorsal appendage short, sinuate, arising subapically from sclerotized ridge. Aedeagus (Figs. 10-M, N) with shaft compressed, bearing two long, robust preapical lateral processes curved ventrolaterad; posteroventral angle of shaft adjacent to gonopore clothed with minute, hairlike spines. Style apex (Fig. 10-P) footlike with distinct heel. Material examined. Holotype $\sigma^{7}$, PERU: Madre de Dios, Rio Tambopata Res. $30 \mathrm{~km}$ (air) SW Pto. Maldonado, 290 m. 12 $2^{\circ} 50$ 'S, $069^{\circ} 17^{\prime} \mathrm{W}$, Smithsonian Institution Canopy Fogging Project, T. L. Erwin, et al. colls., 07 Nov 1983 01/02/48 [USNM]; 1 ठ same data except 14 Sep 1984 01/02/73

[USNM].

Note. The species name refers to the presence of numerous small hairlike spines on the aedeagus.

\section{Spinigina quadrispinosa, new species}

$$
\text { (Figs. 5-N; 10-Q-T) }
$$

Diagnosis. Length of male $3 \mathrm{~mm}$. Male $2 \mathrm{~S}$ apodemes large, rounded, extended beyond posterior margin of sternite III. Pygofer (Fig. 5-N) with dorsal appendage extended to aedeagus (Figs. 10-M, O) with dorsal subbasal hump; pair of slender preapical processes extended ventrad then bent anterad; second pair extended dorsad and curved anterolaterad; unpaired distal process arched over apex from left to right. Style apex (Fig. 10-Q) sicklelike, apex without distinct heel.

Material examined. Holotype $\sigma^{\top}$, PERU: Madre de Dios, Rio Tambopata Res. $30 \mathrm{~km}$ (air) SW Pto. Maldonado, 290 m. 12 50 'S, $069^{\circ} 17^{\prime} \mathrm{W}$, Smithsonian Institution Canopy Fogging Project, T. L. Erwin, et al. colls., 06 Sep 1984 05/02/156 [USNM].

Note. The species name refers to the four paired lateral spines of the aedeagal shaft.

\section{CONCLUDING REMARKS}

Although the New World fauna of Erythroneurini comprises more than 700 described species and many more await discovery and description, the fauna appears to be less phyletically diverse than that of the Old World. The color patterns exhibited by North American species are remarkably varied, but other aspects of the morphology, particularly the structure and chaetotaxy of the male genital capsule and the shape of the connective and styles, are more conservative in the New World fauna. In particular, the connective is $\mathrm{U}$ - or $\mathrm{V}$-shaped in all known New World species, in contrast to the multitude of forms found among Old World species, a majority of which have a median anterior lobe between the two lateral arms. The subgenital plates in nearly all known New World species have an angulate basolateral projection (present in many but not all Old World genera) and a basal submarginal row of three macrosetae. The pygofer appendages are limited to a few basic forms, with most New World species having the dorsal appendage immovably fused to the pygofer side (in contrast to the Old World, in which the vast majority of species appear to have movably articulated dorsal appendages). Erythroneura and the taxa formerly included as 
its subgenera have the style apex with a third point of various forms, but the more basal parts of the style are conservative in the New World fauna; and, with the exception of a few South American species, species of genera endemic to the Neotropical and western Nearctic regions nearly all have a simple, truncate style apex. The depauperate erythroneurine fauna of South America, evidenced by the lack of previously described species from that continent, appears to be confirmed by recent canopy fogging in Amazonia, as well as Malaise trap sampling in Andean premontane forests, and in the more arid grasslands and deserts of Chile and Argentina (unpublished data). Such samples contain an abundance of individuals and species of the typhlocybine tribes Dikraneurini, Empoascini, and Alebrini, but very few Erythroneurini. South American tropical and subtropical grasslands and savannas have not yet been sampled intensively for leafhoppers and these biomes may yet yield diverse erythroneurine taxa, as do similar habitats in Asia and Africa. In contrast, Mexico appears to harbor a rich, but largely undescribed erythroneurine fauna. 


\section{LITERATURE CITED}

Ahmed, M. 1970a. Studies of the genus Erythroneura Fitch (Erythroneurini: Cicadellidae) in West Pakistan. Pakistan Journal of Zoology 2(1):29-42.

Ahmed, M. 1970b. Further studies on the leafhoppers of tribe Erythroneurini (Cicadellidae: Typhlocybinae) from west Pakistan. Pakistan Journal of Zoology 2(2):167-184.

Ahmed, M. 1971b. Studies on the genera and species of tribe Erythroneurini (Cicadellidae, Typhlocybinae) in east Pakistan. Pakistan Journal of Zoology 3(2):175-192.

Ahmed, M. 1985c. Typhlocybinae of Pakistan. Fauna of the subfamily Typhlocybinae (Cicadellidae: Homoptera: Insecta). Pakistan Agricultural Research Council. Islamabad. 279 pp.

Ahmed, M., and K. Samad. 1980a. Erythroneurine leafhoppers of fruit and vegetable plants in Pakistan. Part I. Genus Zyginidia Haupt. Pakistan Journal of Zoology 12(1):93-98.

Anufriev, G.A. 1969b. New and little known leafhoppers of the subfamily Typhlocybinae from the Soviet Maritime Territory (Homopt., Auchenorrhyncha). Acta Faunistica Entomologica Musei Nationalis Pragae 13(153):163-190.

Anufriev, G.A. 1969c. Zyczacella, new subgenus of Erythroneura Fitch (Homoptera: Cicadellidae). Bulletin de l'Academie Polaneise des Sciences. (Serie des Sciences Biologiques) 17(11-12):697700.

Anufriev, G.A. 1971e. Six new far eastern species of leafhoppers (Homoptera, Auchenorrhyncha). Bulletin de l'Academie Polaneise des Sciences. (Serie des Sciences Biologiques) 19(7-8):517522.

Auten, M., and D.M. Johnson. 1936a. Some Erythroneura of the obliqua group from Decatur, Georgia (Homoptera, Cicadellidae). Annals of the Entomological Society of America 29:61-65.

Backus, E.A., M.S. Serrano, and C.M. Ranger. 2005. Mechanisms of hopperburn: an overview of insect taxonomy, behavior, and physiology. Annual Review of Entomology 50:125-151.

Baker, C.F. 1903d. New Typhlocybini. Invertebrata Pacifica 1:9-12.

Baker, C.F. 1925b. Nomenclatorial notes on the Jassoidea, IV. The Philippine Journal of Science 27:537.

Baker, C.F. 1926a. Nomenclatorial notes on the Jassoidea, V. The Philippine Journal of Science 30:347.
Beamer, R.H. 1927a. New species of Erythroneura (Homoptera: Cicadellidae). Canadian Entomologist 59:30-31.

Beamer, R.H. 1929b. Erythroneura (Homoptera, Cicadellidae) from the Southwest. Annals of the Entomological Society of America 22(1):115-129.

Beamer, R.H. 1930a. Two Erythroneura (grape leaf hoppers) damaging apple in Kansas. Journal of the Kansas Entomological Society 3(2):49-50.

Beamer, R.H. 1930b. Some Erythroneura of the obliqua group (Homoptera, Cicadellidae). Annals of the Entomological Society of America 23:417-456.

Beamer, R.H. 1931a. Some Erythroneura (grape leaf hoppers) of the maculata group (Homoptera, Cicadellidae). Canadian Entomologist 63:127135.

Beamer, R.H. 1931b. Some Erythroneura (grape leaf hoppers) of the maculata group (Homoptera, Cicadellidae). Canadian Entomologist 63:240244.

Beamer, R.H. 1931c. Some Erythroneura (grape leaf hoppers) of the maculata group (Homoptera, Cicadellidae). Canadian Entomologist 63:268270.

Beamer, R.H. 1931d. Some Erythroneura (grape leaf hoppers) of the maculata group (Homoptera, Cicadellidae). Canadian Entomologist 63:285289.

Beamer, R.H. 1932a. Some Erythroneura (grape leaf hoppers) of the maculata group (Homoptera, Cicadellidae). Canadian Entomologist 64:12-17.

Beamer, R.H. 1932b. Erythroneura collected on apple with description of a new species. Journal of the Kansas Entomological Society 5(2):62-64.

Beamer, R.H. 1932c. Some Erythroneura (grape leaf hoppers) of the maculata group (Homoptera, Cicadellidae). Canadian Entomologist 64:45-48.

Beamer, R.H. 1932d. Some Erythroneura (grape leaf hoppers) of the maculata group (Homoptera, Cicadellidae). Canadian Entomologist 64:69-72.

Beamer, R.H. 1932e. Some Erythroneura (grape leaf hoppers) of the maculata group (Homoptera, Cicadellidae). Canadian Entomologist 64:82-88.

Beamer, R.H. 1932f. Some Erythroneura (grape leaf hoppers) of the maculata group (Homoptera, Cicadellidae). Canadian Entomologist 64:134144.

Beamer, R.H. 1932g. Some Erythroneura (grape leaf hoppers) of the maculata group (Homoptera, Cicadellidae). Canadian Entomologist 64:158162. 
Beamer, R.H. 1932h. Some Erythroneura (grape leaf hoppers) of the maculata group (Homoptera, Cicadellidae). Canadian Entomologist 64:174181,3 pl.

Beamer, R.H. 1932i. Some Erythroneura from the Western United States (Homoptera, Cicadellidae). Journal of the Kansas Entomological Society 5(4):123-127.

Beamer, R.H. 1932j. A new Erythroneura related to Erythroneura rubricata (Van D.) (Homoptera, Cicadellidae). Pan-Pacific Entomologist. 8(4):183-184.

Beamer, R.H. 1934a. A new genus and two new species of leafhoppers from California (Homoptera Cicadellidae). Pan-Pacific Entomologist 10(1):43-44.

Beamer, R.H. 1934b. Notes on leafhoppers (Homoptera, Cicadellidae). Canadian Entomologist 66:16-18.

Beamer, R.H. 1934c. Notes on some western Erythroneura with description of three new species (Homoptera: Cicadellidae). Journal of the New York Entomological Society 42: 285-288.

Beamer, R.H. 1934d. Two Erythroneura (Homoptera, Cicadellidae). Journal of the Kansas Entomological Society 7:96-97.

Beamer, R.H. 1935a. Ten new species of Erythroneura (Homoptera, Cicadellidae). Journal of the Kansas Entomological Society 8(3):98-104.

Beamer, R.H. 1937a. Five new species of leafhoppers (Homoptera - Cicadellidae). Journal of the Kansas Entomological Society 10(1):10-13.

Beamer, R. H. 1937b. Three new western species of Erythroneura (Homoptera - Cicadellidae). Annals of the Entomological Society of America. 30(1): 31-33.

Beamer, R.H. 1938a. Species of Erythroneura of the comes group (Homoptera - Cicadellidae). The University of Kansas Science Bulletin, 1936, 24(14):261-307.

Beamer, R.H. 1939a. Four new species of leafhoppers and notes on two others (Homoptera, Cicadellidae). Journal of the Kansas Entomological Society 12(1):26-30.

Beamer, R.H. 1941a. Two new species of Erythroneura (Homoptera - Cicadellidae). Journal of the Kansas Entomological Society 14(1):18-19.

Beamer, R.H. 1946b. The Erythroneura of the vulnerata group (Homoptera - Cicadellidae). Journal of the Kansas Entomological Society 19(1):15-22.

Beamer, R.H., and M.E. Griffith. 1935a. New Erythroneura of the obliqua group (Homoptera, Cicadellidae). Journal of the Kansas Entomological Society 8(1):17-21.
Bremer, K. 1994. Branch support and tree stability. Cladistics 10:295-304.

Cerutti, N. 1939a. Les Typhlocybidae du Valais. Bulletin de la Murithienne 56:81-95.

China, W.E. 1930a. A new species of Erythroneura (Homoptera: Jassoidea) injurious to cassava in east Africa. Bulletin of Entomological Research 21:267-268

China W.E. 1931a. A new species of Erythroneura (Homoptera, Jassoidea) injurious to Cassava in east Africa Bulletin of Entomological Research 22:53-54.

China W.E. 1938c. Die Arthropodenfauna von Maderia nach den Ergebnissen der Reise von Prof. Dr. O. Lundblad Juli-August 1935. III. Terrestrial Hemiptera (Hemiptera and Homoptera Auchenorrhyncha). Arkiv för Zoologi 30A(2):168.

Cumber, R.A. 1952a. A new species of Erythroneura (Typhlocybinae. Hem. - Hom.) from Arundo conspicua Forst. (Toetoe). Transactions of the Royal Society of New Zealand 79:525-527.

DeLong, D.M. 1916a. The leafhoppers or Jassoidea of Tennessee. Bulletin of the Tennessee State Board of Entomology 5(2:17):1-113.

DeLong, D.M., and D.J. Knull. 1946. Check list of the Cicadellidae (Homoptera) of America, north of Mexico. Ohio State University Graduate School Studies, Biological Science Series 1:1-102.

Dlabola, J. 1952b. Einige neue paläarktische Zikaden und andere faunistische Bemrekungen. Acta Entomologica Musei Nationalis Pragae 28:27-37.

Dlabola, J. 1957a. Results of the Zoological Expedition of the National Museum in Prague to Turkey. 20. Homoptera, Auchenorrhyncha. Acta Entomologica Musei Nationalis Pragae 31:19-68.

Dlabola, J. 1957b. Die Zikaden Afghanistans (Homopt.-Auchenorrhyncha) nach der Ergebnisse der von Herrn J. Klapperich in den Jahren 1952-1953 nach Afghanistan untergenommenen expedition. Mitteilungen der Muenchen Entomologischen Gesellschaft 47:265-303.

Dlabola, J. 1957c. The problem of the genus Delphacodes and Calligypona, three new species and other Czechoslovakian faunistics (Homoptera, Auchenorrhyncha). Sbornik Entomologickeho Oddeleni Narodniho Musea V Praze 31(476):113119.

Dlabola, J. 1958c. A reclassification of Palaearctic Typhlocybinae (Homopt., Auchenorrh). Casopsis Ceskoslovenske Spolecnosti Entomologicke 55:44-57. 
Dlabola, J. 1961b. Die Zikaden von Zentralasien, Dagestan und Transkaukasien (Homopt. Auchenorrhyncha). Acta Entomologica Musei Nationalis Pragae 34:241-358.

Dlabola, J. 1963a. Zwei neue Erythroneura-Arten an der Weinrebe (Homoptera, Typhlocybinae). Reichenbachia 1(36):309-313.

Dlabola, J. 1968a. Ergebnisse der zoologischen Forschungen von Dr. Z. Kaszab in der Mongolei. Nr. 163: Homoptera, Auchenorrhyncha. Acta Entomologica Bohemoslovaca 65(5):364-374.

Dmitriev, D.A., and C.H. Dietrich. 2006a. Nomenclatural changes and notes in the tribe Erythroneurini (Homoptera: Cicadellidae: Typhlocybinae). Zootaxa 1120:35-39.

Duso, C., A. Bressan, L. Mazzon, and V. Girolami. 2005. First record of the grape leafhopper Erythroneura vulnerata Fitch (Hom., Cicadellidae) in Europe. Journal of Applied Entomology 129:170.

Dworakowska, I. 1972g. Zyginoides Mats. and some other Typhlocybinae (Auchenorrhyncha, Cicadellidae). Bulletin de l'Academie Polonaise des Sciences. (Serie des Sciences Biologiques) 20(12):857-866.

Dworakowska, I. 1970a. A new subgenus of Erythroneura Fitch (Auchenorrhyncha, Cicadellidae, Typhlocybinae). Bulletin de l'Academie Polonaise des Sciences, Serie des Sciences Biologiques 18:347-354.

Dworakowska, I. 1970b. On the genus Arboridia Zachv. (Auchenorrhyncha, Cicadellidae, Typhlocybinae). Bulletin de l'Academie Polonaise des Sciences, Serie des Sciences Biologiques 18:607-615.

Dworakowska, I. 1970c. On the genus Zygina Fieb. and Hypericella sgen. n. (Auchenorrhyncha, Cicadellidae, Typhlocybinae). Bulletin de l'Academie Polonaise des Sciences, Serie des Sciences Biologiques 18:559-557.

Dworakowska, I. 1970d. A new subgenus of Erythroneura Fitch (Auchenorrhyncha, Cicadellidae, Typhlocybinae). Bulletin de l'Academie Polonaise des Sciences. (Serie des Sciences Biologiques) 18(6):347-354.

Dworakowska, I. 1972b. On some Oriental Erythroneurini. Bulletin de l'Academie Polonaise des Sciences. (Serie des Sciences Biologiques) 20(6):395-405.

Dworakowska, I. 1979. On some Erythroneurini from Vietnam (Typhlocybinae, Cicadellidae). Annotationes Zoologicae et Botanicae 131:1-50.
Dworakowska, I. 1980. On some Typhlocybinae from India (Homoptera, Auchenorrhyncha, Cicadellidae). Entomolische Abhandlungen Staatliches Museum für Tierkunde in Dresden 43:151-201.

Dworakowska, I., and C. A. Viraktamath. 1975. On some Typhlocybinae from India (Auchenorrhyncha, Cicadellidae). Bulletin de l'Academie Polonaise des Sciences, Serie des Sciences Biologiques 23:521-530.

Emeljanov, A.F. 1964f. New Auchenorrhyncha from Kazakhstan (Homoptera). Trudy Zoologicheskogo Instituta Akademii Nauk SSR 34:3-51. (In Russian).

Esaki, T., and S. Ito. 1954a. A tentative catalogue of Jassoidea of Japan, and her adjacent territories. Tokyo: Japan Soc. Promot. Sci. 315 pp.

Evans, J.W. 1940c. Some Queensland leaf-hoppers (Jassoidea, Homoptera) that attack lucerne. Proceedings of the Royal Society of Queensland 52(2):10-13.

Evans, J.W. 1948a. A new species of Erythroneura from Fiji (Homoptera: Jassidae). Bulletin of Entomological Research 39:131

Fairbairn, V.M. 1928b. The genus Hymetta (Homoptera, Cicadellidae). Journal of the Kansas Entomological Society 1(4):84-92.

Fieber, F.X. 1866a. Neue Gattungen und Arten in Homoptern (Cicadina Bur.). ZoologischeBotanische Gesellschaft Wiener Verhandlungen 16:497-516.

Fitch, A. 1851a. Catalogue with references and descriptions of the insects collected and arranged for the State Cabinet of Natural History. Annual report of the Regents of the University on the condition of the State Cabinet of Natural History 4:43-69.

Fitch, A. 1956a. Third report on noxious and other insects of the State of New York. Transactions of the New-York State Agricultural Society 16:315490, pl. 1-4.

Günthart, H. 1974a. Beitrag zur Kenntnis der Kleinzikaden (Typhlocybinae, Hom., Auch.) der Schweiz. 1. Erganzung. Mitteilungen der Schweizerischen Entomologischen Gesellschaft 47(1-2):15-27.

Gillette, C.P. 1892a. Observations upon injurious insects, season of 1891. Bulletin of the Colorado Agricultural Experimental Station 19:1-32.

Gillette, C.P. 1898a. American leaf-hoppers of the subfamily Typhlocybinae. Proceedings of the United States National Museum 20(1138):709_ 773. 
Hamilton, K.G.A. 1983. Introduced and native leafhoppers common to the Old and New Worlds (Rhynchota: Homoptera: Cicadellidae). Canadian Entomologist 115:473-511.

Hamilton, K.G.A. 1985. Leafhoppers of ornamental and fruit trees in Canada. Publication 1779/E, 71 pp., Agriculture Canada, Ottawa.

Hamilton, K.G.A., and D.W. Langor. 1987a. Leafhopper fauna of Newfoundland and Cape Breton islands (Rhynchota: Homoptera: Cicadellidae). Canadian Entomologist 119(7-8):663-695.

Harris, T.W. 1831a. Locust. Encyclopaedia Americana. A popular dictionary of arts, sciences, literature, history, politics, and biography, brought down to the present time; including a copious collection of original articles in American biography... 8:40-43.

Heller, F., and R. Linnavuori. 1968a. Cicadelliden aus Äthiopien. Stuttgarter Beiträge zur Naturkunde. (Serie A, Biologie) 186:1-42.

Hepner, L.W. 1966a. Twenty species of Erythroneura related to E. bigemina (Homoptera: Cicadellidae). Journal of the Kansas Entomological Society 39(1):78-89.

Hepner, L.W. 1966b. New species of Erythroneura related to lenta (Homoptera: Cicadellidae). Florida Entomologist 49(2):95-100.

Hepner, L.W. 1966c. New species of Erythroneura (Cicadellidae) related to inepta. Journal of the Georgia Entomological Society 1(4):1-5.

Hepner, L.W. 1966d. New species of Erythroneura related to campora (Homoptera: Cicadellidae). Florida Entomologist 49(2):101-106.

Hepner, L.W. 1967a. New species of Erythroneura related to E. dira (Homoptera: Cicadellidae). Journal of the Kansas Entomological Society 40(1):17-24.

Hepner, L.W. 1967b. New species of Erythroneura (Homoptera: Cicadellidae). Entomological News 78(3):59-73.

Hepner, L.W. 1969a. New species of Erythroniura from oaks and hickories (Homoptera: Cicadellidae). Journal of the Kansas Entomological Society 42(2):126-133.

Hepner, L.W. 1972a. Five new species of Erythroneura (Homoptera: Cicadellidae). Journal of the Kansas Entomological Society 45(4):430-433.

Hepner, L.W. 1972b. A new species of Erythroneura (Homoptera: Cicadellidae) and some characteristics of the nymph. Journal of the Georgia Entomological Society 7(3):216-218.

Hepner, L.W. 1972c. New species of Erythroneura (Homoptera: Cicadellidae). Florida Entomologist 55(4):267-272.
Hepner, L.W. 1973a. New species of Erythroneura (Homoptera: Cicadellidae). Journal of the Kansas Entomological Society 46(2): 184-186.

Hepner, L.W. 1975a. New species of Erythroneura (Homoptera: Cicadellidae). Journal of the Kansas Entomological Society 48(1):4-7.

Hepner, L.W. 1976a. Thirteen new species of Erythroneura (Erythridula) (Homoptera: Cicadellidae). Journal of the Kansas Entomological Society 49(2):204-211.

Hepner, L.W. 1976b. Sixteen new species of Erythroneura (Erythridula) from Eastern North America (Homoptera, Cicadellidae). III. Journal of the Georgia Entomological Society 11(2):119126.

Hepner, L.W. 1976c. Fifteen new species of Erythroneura (Erythridula) (Homoptera, Cicadellidae). II. Florida Entomologist 59(3):293-300.

Hepner, L.W. 1976d. Seventeen new species of Erythroneura (Erythridula) (Homoptera, Cicadellidae). VI. Journal of the Georgia Entomological Society 11(4):309-316.

Hepner, L.W. 1977a. Fourteen new species of Erythroneura (Erythridula) (Homoptera: Cicadellidae). IV. Journal of the Kansas Entomological Society 50(2):247-255.

Hepner, L.W. 1977b. Fourteen new species of Erythroneura (Erythridula) (Homoptera: Cicadellidae). V. Florida Entomologist 60(1):49-56.

Hepner, L.W. 1977c. Fifteen new species of Erythroneura (Erythridula) (Homoptera, Cicadellidae). VIII. Journal of the Georgia Entomological Society 12(4):359-365.

Hepner, L.W. 1978a. Sixteen new species of Erythroneura (Erythridula) (Homoptera, Cicadellidae). VII. Journal of the Kansas Entomological Society 51(1):131-139.

Horváth, G. 1909a. Hémiptères recueillis par M. Th. Becker aux Iles Canaries. Annales Musei Nationalis Hungarici 7:631-632.

Horváth, G. 1910b. Magyarországi új Homoptera. Rovartani Lapok 17:176-177.

Ishihara, T. 1965c. Some species of Formosan Homoptera. Special Bulletin of Lepidopterological Society of Japan 1:201-221.

Izzard, R.J. 1936a. The Hemiptera of Christmas Island. The Annals and Magazine of Natural History (10) 17(102):577-600.

Johnson, D.M. 1934a. Some Erythroneura of the comes group (Homoptera: Cicadellidae). The Ohio Journal of Science 34(4):258-263.

Johnson, D.M. 1935a. Leafhoppers of Ohio. Subfamily Typhlocybinae (Homoptera: Cicadellidae). Bulletin of the Ohio Biological Survey 31:39-122. 
Johnson, D., and M. Authen. 1936a. Omissions and errors. Annals of the Entomological Society of America 34(4):818.

Kirkaldy, G.W. 1906a. An historical note on the parasitism of certain Homoptera. Entomologist 39:14.

Kirkaldy, G.W. 1906c. Leafhoppers and their natural enemies. (Pt. IX, Leafhoppers. Hemiptera. Bulletin of the Experiment Station of the Hawaiian Sugar Planters' Association. (Entomological series) 1(9):271-479.

Kirkaldy, G.W. 1907d. Leafhoppers supplement (Hemiptera). Bulletin of the Experiment Station of the Hawaiian Sugar Planters' Association. (Entomological series) 3:1-186, pl. 1-20.

Knull, D.J. 1944b. Nomenclatorial notes on Cicadellidae. Annals of the Entomological Society of America 37:123.

Knull, D.J. 1945b. Eleven new leafhoppers with notes on others (Homoptera: Cicadellidae). The Ohio Journal of Science 45(3):103-110.

Knull, D.J. 1946a. Erythroneura of the obliqua group from Ohio and Tennessee (Homoptera: Cicadellidae). The Ohio Journal of Science 46(1):45-49.

Knull, D.J. 1949a. New leafhoppers from the United States (Homoptera: Cicadellidae). The Ohio Journal of Science 49:119-126.

Knull, D.J. 1951b. Sixteen new leafhoppers and notes (Homoptera: Cicadellidae). The Ohio Journal of Science 51(4):169-178.

Knull, D.J. 1951c. Eight new leafhoppers from the United States (Homoptera: Cicadellidae). The Ohio Journal of Science 51(4):179-183.

Knull, D.J. 1954b. New Erythroneura (Eratoneura) of the dira group with notes (Homoptera, Cicadellidae). The Ohio Journal of Science 54(3): 170-174.

Knull, D.J. 1954d. A Frigartus from California and an Erythroneura from South Dakota (Homoptera: Cicadellidae). Entomological News 65(2):37-39.

Knull, D.J. 1955a. Some new texan Erythroneura (Eratoneura) of the dira group (Homoptera: Cicadellidae). The Ohio Journal of Science 55(4):245-246.

Knull, D.J., and M. Auten. 1937a. Some Erythroneura of the maculata group from Decatur, Georgia (Homoptera: Cicadellidae). Annals of the Entomological Society of America 30:572-578.

Knull, D.J., and M. Auten. 1938a. Some Erythroneura from the Southwest (Homoptera: Cicadellidae). Annals of the Entomological Society of America 31:532-539.
Kusnetsov, V. 1928b. Deux Cicadellides nuisebles due genre Erythroneura Fitch (Homoptères). Defense des Plantes 5:315-317.

Kusnetsov, V. 1932a. Zwei neue schädliche Homopteren aus Zentral-Asien. Wiener entomologische Zeitung 48:112-114.

Lang, V. 1945c. Zlomky z cikadologichého výzkumu mohelnské reservace. Paté příspévek k poznání našich cikád. Folia Entomologica Hungarica 8:95-99.

Lindberg, H. 1936a. Die Cicadinen der Kanarischen Inseln. Commentationes Biologicae Societas Scienterium Fennica 6(9):1-19.

Lindberg, H. 1948b. On the insect fauna of Cyprus. Results of the expedition of 1939 by Harald Håkan and P.H. Lindberg. II. Heteroptera und Homoptera Cicadina der Insel Zypern. Commentationes Biologicae Societas Scienterium Fennica 10(7):23-175.

Lindberg, H. 1954a. Hemiptera Insularum Canariensium. Systematik, Ökologie und Verbreitung der Kanarischen Heteropteren und Cicadinen. Commentationes Biologicae Societas Scienterium Fennica 14(1):1-304.

Lindberg, H. 1961a. Hemiptera Insularum Madeirensium. Commentationes Biologicae Societas Scienterium Fennica 24(1):1-82.

Linnavuori, R. 1953d. On some new or interesting leafhopper species of the family Cicadellidae. Annales Zoologici Fennici 19:56-63.

Linnavuori, R. 1956b. On some Palearctic Homoptera. Suomen Hyonteistieteellinen Aikakaukirja 22:136-138.

Linnavuori, R. 1960a. Insects of Micronesia. Homoptera: Cicadellidae. Honolulu, Bishop Museum 6(5):231-344.

Linnavuori, R. 1960b. Cicadellidae (Homoptera, Auchenorrhyncha) of Fiji. Acta Entomologica Fennica 15:1-71.

Linnavuori, R. 1962a. Hemiptera of Israel. III. Suomalaisen Elain-ja Kasviteiteellisen Seuran vanamon Elaintieteellsia Julkaisuja 24(3):1-108.

Linnavuori, R. 1964a. Hemiptera of Egypt, with remarks on some species of the adjacent Eremian region. Annales Zoologici Fennici 1:306-356.

Linnavuori, R. 1965a. Studies on the south and east Mediterranean hemipterous fauna. Acta Entomologica Fennica 21:5-70.

Matsumura, S. 1931. A revision of the Palaearctic and Oriental typhlocybid genera, with descriptions of new species and new genera. Insecta Matsumurana 6:55-91. 
McAtee, W.L. 1918b. Notes on nova scotian eupteryid leafhoppers including descriptions of two new species. Canadian Entomologist 50:360-361.

McAtee, W.L. 1919a. A new genus for Tettigonia trifasciata Say (Homoptera; Eupterygidae). Proceedings of the Biological Society of Washington 32:121-124.

McAtee, W.L. 1920a. Key to the nearctic species and varieties of Erythroneura (Homoptera; Eupterygidae). Transactions of the American Entomological Society 46:267-321, pl. XII.

McAtee, W.L. 1924c. Notes on eupterygid leafhoppers with descriptions of a few forms (Homoptera). Florida Entomologist 8(3-4):33-39.

McAtee, W.L. 1924d. Records of species of the genus Erythroneura (Homoptera; Eupterygidae) with descriptions of new forms. Proceedings of the Biological Society of Washington 37:131-134.

McAtee, W.L. 1924e. Notes on a collection of Erythroneura and Hymetta (Eupterygidae) chiefly from Illinois, with descriptions of new forms. Bulletin of the Illinois Natural History Survey 15(2):39-44.

McAtee, W.L. 1926c. Notes on Homoptera from Illinois, with descriptions of new forms, chiefly Eupteryginae. Bulletin of the Illinois Natural History Survey 16(3):127-136.

Metcalf, Z.P. 1955a. New names in the Homoptera. Journal of the Washington Academy of Sciences 45:262-267.

Mitjaev, I.D. 1969b. New species of leaf-hoppers (Homoptera, Cicadinea) from Tien Shan and Karatau. Zoologicheskiy Zhurnal. 48(7):10411047. (In Russian).

Mitjaev, I.D. 1971a. Leafhoppers of Kazakhstan (Homoptera-Cicadinea). The diterminant. AlmaAta: Nauka. 210 pp. (In Russian).

Myers, J.G. 1923a. A contribution to the study of New Zealand leaf-hoppers and plant-hoppers (Cicadellidae and Fulgoroidea). Transactions and proceedings of the New Zealand Institute 54:407-429.

Naudé, T.J. 1926a. Cicadellidae of South Africa. A taxonomic and faunistic study. Entomological Memoirs of the Department of Agriculture of the Union of South Africa 1(4):1-106, pls. I-X.

Oman, P.W., W J. Knight, and M.W. Nielson. 1990. Leafhoppers (Cicadellidae): A bibliography and generic check-list and index to the world literature 1956-1985. C.A.B. International Institute of Entomology, Wallingford, Oxon, U.K.

Osborn, H. 1928a. Neotropical Homoptera of the Carnegie Museum. Part 6. Report on the subramily Typhlocybinae, with descriptions of new species. Annals of the Carnegie Museum 18: 253-298, pls. 13-16.
Ossiannilsson, F. 1937a. Zur Kenntnis der schwedischen Homopterenfauna mit Beschreibung der neuen Art Erythroneura silvicola Oss. Opuscula Entomologica 2:19-27.

Provancher, L. 1890a. Deuxième sous-ordre les Homoptères. Petite Faune Entomologique du Canada, precede d'un traiteé élémentaire d'entomologie 3:283-334.

Rakitov, R.A. 1998. On differentiation of cicadellid leg chaetotaxy (Homoptera: Aucenorrhyncha Membracoidea). Russian Entomological Journal $6(3-4): 7-27$.

Ribaut, H. 1931c. Les espèces Françaises des groupes Erythroneura parvula (Boh.) et Erythroneura fasciaticollis (Rey) (Homoptera-Typhlocybidae). Bulletin of the Societe dHostoire Naturelle de Toulouse 62:399-416.

Ribaut, H. 1936b. Homoptères Auchénorhynques. I. (Typhlocybidae). Faune de France. Paris: Lechevalier. 31: III+231 pp.

Ribaut, H. 1948a. On the insect fauna of Cyprus. Results of the expedition of 1939 by Harald Håkan and P.H. Lindberg. III. Homoptères nouveaux de Chypre. Commentationes Biologicae Societas Scienterium Fennica 10(8):3-14.

Ribaut, H. 1952a. Homoptères Auchénorhynques. II. (Jassidae). Faune de France. Paris: Lechevalier. 57:474 pp.

Ribaut, H. 1959b. Nouvelles especes francaises d'Homopteres. Bulletin of the Societe dHostoire Naturelle de Toulouse 94:400-405.

Richards, W.R., and I.W. Varty. 1964a. A new species of Erythroneura Fitch (Homoptera: Cicadellidae). Canadian Entomologist 96(3(:515-516.

Robinson, W. 1924a. Some new species of Erythroneura (Homoptera, Cicadellidae). Canadian Entomologist 56(3):58-62.

Robinson, W. 1924b. Additional new species of Erythroneura (Homoptera, Cicadellidae). Canadian Entomologist 56:154-157.

Robinson, W. 1924c. Additional new species of Erythroneura (Homoptera, Cicadellidae). Canadian Entomologist 56:290-292.

Ross, H.H. 1953b. Polyphyletic origin of the leafhopper fauna of Ilex decidua. Transactions of the Illinois State Academy of Science 16:186-192.

Ross, H.H. 1956a. New nearctic species of Erythroneura (Homoptera: Cicadellidae). Entomological News 67(4):85-90.

Ross, H.H. 1957a. New oak-inhabiting species of Erythroneura from Illinois (Hemiptera, Cicadellidae). Entomological News 68:183-190. 
Ross, H.H. 1965a. The phylogeny of the leafhopper genus Erythroneura (Hemiptera, Cicadellidae). Zoologische Beiträge 11(1-2):247-270.

Ross, H.H., and D.M. DeLong. 1950a. New species of Erythroneura of the maculata group (Homoptera: Cicadellidae). The Ohio Journal of Science 50(6):291-296.

Ross, H.H., and D.M. DeLong. 1953a. Biological and taxonomic notes on Erythroneura (Homoptera, Cicadellidae). The Ohio Journal of Science 53(2):77-90.

Samad, K., and M. Ahmed. 1979b. Erythroneurine leafhoppers of fruit and vegetable plants in Pakistan. Genera Zygina Fieber, Erythroneura Fitch and Jalalia Ahmed. Islamabad Journal of Sciences 6(1-2):1-4.

Say, T. 1825a. Descriptions of new Hemipterous insects collected in the expedition to the Rocky Mountains, performed by order of Mr. Calhoun, Secretary of War, under command of Major Long. Journal of the Academy of Natural Sciences of Philadelphia 4:307-345.

Shcherbakov, D.Y. 1981. Diagnostics of the families of the Auchenorrhyncha (Homoptera) on the basis of the wings. I. Forewing. Entomological Review 60:64-81.

Shcherbakov, D.Y. 1982. Diagnostics of the families of the Auchenorrhyncha (Homoptera) on the basis of the wings. II. Hindwing. Entomological Review 61:70-78.

Sinha, R.N., and R.H. Beamer. 1954a. A new species of Erythroneura (Homoptera: Cicadellidae) from Kansas. Journal of the Kansas Entomological Society 27(3):105.

Sohi, A.S., and I. Dworakowska. 1984. A review of the Indian Typhlocybinae (Homoptera: Cicadellidae) from India. Oriental Insects 17:159-213.

Sohi, A.S., and V.C. Kapoor. 1974a. A new species of the genus Erythroneura Fitch (Homoptera: Cicadellidae) from India. Indian Journal of Entomology 36(1):42-43.

Swofford, D.L. 1998. PAUP*: Phylogenetic Analysis Using Parsimony (*and Other Methods), Version 4. Sinauer, Sunderland, Massachusetts.

Van Duzee, E.P. 1909a. Observation on some Hemiptera taken in Florida in the spring of 1908. Bulletin of the Buffalo Society of Natural Sciences 9:149-230.

Van Duzee, E.P. 1924a. The genus Erythroneura in California (Homoptera). Proceedings of the California Academy of Sciences. (4) 13(13):231-236.

Vilbaste, J. 1968a. Uber die Zikadenfauna des Primorje Gebietes. Tallin: Valgus. 195 pp. (In Russian).
Walsh, B.D. 1862a. Fire blight. Two new foes of the apple and pear. The Prairie Farmer 10:147149. (New series.)

Wagner, W. 1939a. Die Zikaden des Mainzer Beckens. Zugleich eine revision der Kirschbaumschen Arten aus der umgebung von Wiesbade. Jahrbücher des Nassauischen Vereins für Naturkunde 86:77-212.

Wagner, W. 1944b. Erythroneura franzi, eine neue Deutsche Erythroneura-Art aus der ScutellarisGruppe (Homoptera, Typhlocibidae). Mitteilungen aus der Entomologischen Gesellschaft zu Halle 20:38-42.

Young, D.A. 1952. A reclassification of Western Hemisphere Typhlocybinae (Homoptera, Cicadellidae). University of Kansas Science Bulletin 35:1-217.

Zachvatkin, A.A. 1946. Studies on the Homoptera of Turkey. I-VII. Transactions of the Royal Entomological Society of London 97:148-176. 


\section{APPENDIX A Morphological Characters for Phylogenetic Analysis of Erythroneurini}

\section{Head}

1. Width: 0 , narrower than pronotum; 1 , as wide as or wider than pronotum.

2. Crown: 0 , anterior margin strongly produced and angulate medially (Fig. 2-D); 1, anterior margin slightly produced, longer medially than next to eye (Fig. 2-M); 2, weakly produced, anterior and posterior margins parallel.

3. Coronal suture: 0 , extended to crown apex; 1 , incomplete, not reaching crown apex; 2 , absent or very short $(<1 / 4$ distance to crown apex $)$.

4. Ocelli: 0, well developed; 1 , absent or vestigial.

5. Eye: 0 , narrower than vertex width; 1 , as wide as or wider than vertex width.

6. Face in profile: 0 , not depressed, $45^{\circ}$ or more from horizontal; 1 , depressed, less than $45^{\circ}$ from horizontal.

7. Male anteclypeus: 0 , narrow, depressed, as in female; 1 , inflated, broad, in contrast to that of female.

8. Maxillary plate: 0 , not extended laterad of lorum; 1 , expanded laterad of lorum, lorum well separated from lateral margin of face (Fig. 1-A).

\section{Thorax}

9. Pronotum: 0, without conspicuous pits; 1 , with conspicuous pits.

10. Forewing vein $\mathrm{CuA}$ : 0, distinctly shorter than $\mathrm{CuP}$ (Fig. 1-B); 1, subequal to $\mathrm{CuP}$ (Fig. 3-D); 2, distinctly longer than $\mathrm{CuP}$ (Fig. 3-C).

11. Forewing vein CuA: 0, longer than MP; 1, shorter than MP (Fig. 1-B); 2, CuA absent (MP connected to $\mathrm{Cu}$ stem).

12. Forewing inner apical cell: 0 , elongate, parallel-sided through most of its length (Fig. 1-B); 1, wide basally, tapered distally; 2 , very short and oblique.

13. Inner (fourth) apical cell base: 0 , oblique, apex of $\mathrm{Cu}$ and $\mathrm{m}$-cu crossvein forming continuous line (Fig.

3-A); 1, distinctly angulate (Fig. 3-D); 2, transverse (Fig. 1-B).

14. Second apical cell base: 0 , quadrate (Fig. 1-B); 1, petiolate.

15. Third apical cell: 0, widened distally (Fig. 3-P); 1, parallel sided (Fig. 1-B); 2, narrowed distally.

16. Third apical cell: 0, straight (Fig. 1-B); 1, curved (Fig. 3-Q).

17. Outer (first) apical cell: 0 , short, length $<2$ times width (Fig. 3-M); 1, length subequal to 2 times width

(Fig. 1-B); 2, elongate, length distinctly greater than 2 times width (Fig. 3-R).

18. Anal (claval) vein: 0, visible throughout length (Fig. 3-Q); 1, obsolete (Fig. 3-C).

19. Forewing appendix: 0 , absent; 1 , present.

20. Hindwing apex: 0, broadly rounded (Fig. 3-V); 1, distinctly narrowed (Fig. 3-T); 2, truncate (Fig. 3-U).

21. Hindwing submarginal vein: 0 , complete (RA present along costal margin and continuous with submarginal vein at wing apex); 1, extended to apex of RP or RM; 2, absent at wing apex (Fig. 1-C).

22. Hindwing vein RA: 0, present (Fig. 1-C); 1, absent.

23. Hindwing vein RP: 0 , free; 1 , absent or fused to MA (Fig. 1-C).

24. Hindwing veins MP and CuA: 0 , separate, connected by crossvein, or touching at one point (Fig. 3-W); 1 , fused for short distance, then divergent (Fig. 1-C); 2, completely confluent.

25. Hindwing veins MP and CuA: 0, divergent distally (Fig. 1-V); 1, parallel (Fig. 1-C); 2, convergent distally (Fig. 1-U); 3, CuA reduced or absent distally.

26. Hindwing vannal vein (Pcu+A1): 0, branched (Pcu and A free distally); 1, unbranched (Pcu and A completely confluent; Fig. 1-C).

27. Front femur AM1 seta: 0, absent or undifferentiated (Fig. 1-D); 1, enlarged, on ventral margin.

28. Front femur AV: 0 , setae subequal; 1 , with two or more basal setae enlarged; 2 , one basal seta distinctly larger than others (Fig. 1-D).

29. Front femur PV: 0, without fine basal setae; 1, with fine basal setae.

30. Middle femur: 0 , with 2 dorsoapical macrosetae; 1 , with 1 dorsoapical macroseta.

31. Hind tibia AV: 0 , with 4 macrosetae; 1 , with 5 macrosetae; 2 , with 6 macrosetae; 3 , with 7 or more macrosetae.

\section{Male abdomen}

32. 2S abdominal apodemes: 0, small, narrow, extended dorsomesad (Fig. 7-M); 1, large, broad, extended to or near 3S posterior margin (Fig. 9-K); 2, large, broad, extended beyond 3S posterior margin.

33. Pygofer length relative to subgenital plate: 0 , shorter (Fig. 1-E); 1, subequal; 2, longer.

34. Pygofer dorsoapical lobe: 0, rounded (Fig. 4-B); 1, angulate (Fig. 4-I); 2, acutely produced. 
35. Tergite IX emargination: 0, extended to base of segment (Fig. 7-N); 1, extended ca. halfway to base of ment (Fig. 7-P); 2, shallow, not extended halfway to base of segment.

36. Pygofer dorsal membrane: 0 , without fine setae; 1 , with conspicuous fine setae.

37. Pygofer dorsal macrosetae: 0 , absent; 1 , one or two present (Fig. 5-B); 2, several present.

38. Pygofer ventroapical membranous area: 0 , absent or inconspicuous; 1 , well developed (Fig. 4-C).

39. Pygofer oblique dorsolateral internal ridge: 0 , absent; 1, present (Fig. 4-F).

40. Pygofer basolateral setae: 0, undifferentiated; 1, in distinct group, small (Fig. 4-A); 2, distinctly enlarged (Fig. 1-E); 4, long and fine.

41. Pygofer distal setae: 0 , undifferentiated; 1 , in distinct group.

42. Pygofer long fine setae: 0 , absent; 1 , present, sparse; 2 , numerous, conspicuous.

43. Pygofer microtrichia: 0, absent or inconspicuous; 1 , well developed, at least distally.

44. Pygofer dorsal appendage: 0 , movably articulated, ventral angle of base free (Fig. 4-A); 1, with distinct basal suture, but not movably articulated; 2, fused, without suture (Fig. 1-E); 3, absent (Fig. 4-I).

45. Pygofer dorsal appendage shape: 0 , simple (Fig. 1-E); 1, simple, with additional small process at base; 2, bifurcate far from base (Fig. 4-N); 3, bifurcate near base, branches parallel; 4, bifurcate near base, branches widely separated (Fig. 4-C).

46. Pygofer appendage: 0 , without small basal internal process; 1 , with small basal internal process.

47. Pygofer dorsal appendage length: 0 , not reaching pygofer apex; 1 , reaching pygofer apex; 2 , extended beyond pygofer apex.

48. Pygofer appendage dorsal branch: 0 , subequal to ventral branch in length; 1 , shorter than ventral branch in length.

49. Pygofer dorsal appendage, dorsal view: 0 , straight or very slightly curved; 1 , distinctly sinuate.

50. Pygofer dorsal appendage, lateral view: 0 , straight; 1 , curved upward; 2 , curved downward.

51. Pygofer ventral appendage: 0 , present (Fig. 1-E); 1, absent.

52. Sternite IX median longitudinal internal ridge: 0 , absent; 1 , present.

53. Subgenital plate lateral margin: 0 , rounded; 1 , straight; 2 , distinctly widened subbasally; 3 , with angulate subbasal projection (Fig. 6-A); 4, with distinct process.

54. Subgenital plate proportions: 0 , section basad of medial constriction subequal to or shorter than distal section; 1 , basal section longer than distal section.

55. Subgenital plate lateral subdistal fold: 0 , absent or indistinct; 1 , distinct, angulate.

56. Subgenital plate basal macrosetae: 0, absent; 1, one; 2, two-four (Fig. 1-I); 3, five-seven; 4, nine or more. 57. Subgenital plate macrosetal row: 0 , uniseriate, along margin; 1 , oblique, extended posteromesad; 2 , scattered.

58. Subgenital plate marginal subbasal setae: 0 , absent or poorly differentiated; 1 , distinct, peglike, forming continuous row (Fig. 6-A); 2, peglike setae restricted to basolateral angle; 3, group of macrosetae present; 4, long fine setae present.

59. Subgenital plate distal macrosetae: 0 , absent; 1 , present.

\section{Genitalia}

60. Style preapical lobe: 0 , absent or weak; 1, prominent (Fig. 1-H); 2, cheliform; 3, greatly enlarged (Fig. 6-S); 4, acuminate; 5 , auriculate.

61. Style apex: 0, smooth; 1 , serrate; 2, crenulate (Fig. 1-I).

62. Style apex: 0, slender (Fig. 10-B); 1, truncate and expanded (footlike) (Fig. 1-I); 2, with 3 points (Fig. $1-\mathrm{H})$.

63. Style second point: 0 , present only as angle or small tooth; 1, well developed (Fig. 1-H); 2, longer than third point. This and the following two characters are applicable only to those having the style apex with three points.

64. Style third point (apical extension): 0, reduced to small tooth (Fig. 7-A); 1, subequal to other points; 2, larger than but shorter than half distance between other points; 3 , longer than half distance between other two points (Fig. 1-H); 4, as long as or longer than distance between other two points; 5, extremely elongate, curved mesad (Fig. 6-Q).

65. Angle between first and third point: 0 , unknown (third point very short); 1, ca. $90^{\circ} ; 2,<90^{\circ} ; 3,>90^{\circ}$. 66. Aedeagus/connective articulation: 0 , at apex of connective stem; 1 , near connective fork (stem overlapping base of aedeagus).

67. Aedeagus dorsal apodeme, lateral view: 0 , absent; 1, present, not expanded in lateral view (Fig. 6-B); 2, broadly expanded in lateral view (Fig. 1-F). 
68. Aedeagus dorsal apodeme, posterior view: 0 , very short or long and parallel-sided, connections to pygofer membranous; 1 , with U- or V-shaped dorsolateral ligaments connected to anal tube and/or pygofer appendage (Fig. 6-H); 2, triangular or T-shaped, without distinct connection to anal tube or pygofer appendage (Fig. $1-\mathrm{G})$.

69. Aedeagus preatrium: 0, short; 1, elongate (Fig. 1-F).

70. Aedeagal shaft in posterior view: 0 , symmetrical; 1 , asymmetrical.

71. Aedeagal shaft, crossection: 0 , round; 1 , depressed; 2 , compressed.

72. Aedeagal shaft in lateral view: 0 , slender; 1 , broad.

73. Aedeagus apex in posterior view: 0 , broadened; 1 , slender, truncate; 2 , acuminate.

74. Aedeagal shaft: 0 , smooth; 1 , denticulate distally.

75. Aedeagus dorsal process between phallobase and gonopore-bearing shaft: 0 , absent; 1, present (Fig. 9-A). 76. Aedeagus preapical processes: 0 , present basally, well separated from shaft, longer than shaft; 1 , present basally, well separated from shaft, shorter than shaft; 2, present basally, close to shaft, about as long as shaft; 3 , present basally, close to shaft, much shorter than shaft; 4 , two pairs of processes present; 5 , arising near midlength of shaft; 6 , arising near apex of shaft; 7 , with unpaired ventral process.

77. Ventral processes, relative position: 0 , evenly divergent; 1 , divergent at base, then parallel; 2 , slightly divergent, appressed to sides of aedeagal shaft; 3 , parallel to each other on ventral side of shaft; 4 , divergent only at apex; 5 , one pair parallel to shaft, other strongly divergent; 6 , unpaired.

78. Aedeagus paired distal processes: 0, long, apical (Fig. 1-G); 1, short, apical; 2, long, subapical; 3, short, subapical; 4 , absent.

79. Apical processes, shape: 0, slender (Fig. 1-G); 1, small, toothlike; 2, flattened, triangular (Fig. 6-K).

80. Aedeagus dorsal distal lobe: 0 , absent; 1 , small; 2, large (Fig. 1-D).

81. Connective, median anterior lobe: 0, absent (Fig. 1-I); 1, present, broad; 2, present, slender.

82. Connective arms: 0 , short; 1 , long.

83. Connective stem: 0 , absent or very short; 1 , well developed, entire; 2 , bifid.

84. Connective stem: 0 , depressed; 1 , compressed.

85. Anal tube processes: 0 , absent (partially sclerotized aedeagal ligaments may be present); 1 , present only as sclerotized rodlike basolateral arms (Fig. 4-I); 2, separate processes arising from basal apodemes

86. Anal tube vestiture: 0 , without lateral spines (microtrichia may be present); 1 , with numerous lateroapical spines (Fig. 5-N).

\section{Coloration}

87. Ground color of dorsum: 0 , pale yellow or white; 1 , dark reddish or brown.

88. Vertex: 0, unicolorous or with indistinct markings; 1, with pair of dark preapical spots (Fig. 2-I); 2, with large median apical spot (Fig. 2-P); 3, with orange parallel submedial lines (often with lateral branch) (Fig.

2-C); 4, with oblique lateral vittae (Fig. 2-A); 5, with median triangular area extended onto thorax; 6, mostly dark with small pale lines or spots (Fig. 2-D).

89. Vertex midline: 0 , pale; 1 , dark.

90. Face: 0 , without black spots anterodorsad of antennal pits; 1 , with black spots anterodorsad of antennal pits (Fig. 1-A).

91. Anteclypeus: 0, concolorous with rest of face; 1, brown or black, contrasting with pale face (Fig. 1-A).

92. Pronotum: 0, without Y- or V-shaped medial vitta; 1, with Y- or V-shaped medial vitta (Fig. 2-B).

93. Mesonotum: 0, without dark lateral triangles; 1, with dark lateral triangles (Fig. 2-F).

94. Scutellum apex: 0 , concolorous with rest of scutellum; 1 , dark, contrasting with adjacent pale areas (Fig. 2-K).

95. Forewing: 0, without numerous irregular red dots; 1, with numerous irregular red dots (Fig. 2-E).

96. Forewing: 0, without oblique vittae; 1, with vittae forming zigzag pattern (Fig. 3-B); 2, with broken, oblique vittae (Fig. 3-C).

97. Clavus: 0 , unicolorous; 1 , with separate basal and distal vittae; 2 , with continuous vitta parallel to suture;

2 , largely or entirely bright red.

98. Costal margin: 0 , without dark spot; 1 , with dark spot

99. Second apical cell: 0 , without brown apical spot; 1 , with brown apical spot.

100. Inner (fourth) apical cell: 0, without brown spot basally; 1, with brown spot basally (Fig. 3-B). 
All taxa are deposited in INHS unless otherwise indicated.

Outgroups. Dikraneura arizona DeLong and Caldwell; Empoasca sp. C (Jalisco, Mexico); Empoascini, N. Gen. A. (Onkonegare, Orellana, Ecuador) [USNM]; Empoascini, N. Gen. B (Ivmaka Res. Sta., Papua New Guinea); Eupteryx artemisiae (Kirschbaum); Jorumidia sp. (Onkonegare, Orellana, Ecuador) [USNM]; Joruma sp. (Onkonegare, Orellana, Ecuador) [USNM]; Kunzeana sp. (La Reforma, Oaxaca, Mexico); Linnavuoriana roseipennis (Oshanin); Paralebra sp. (ca. Zenzontla, Jalisco, Mexico).

Ingroup Old World Taxa. Asianidia pallescita (Dlabola); Arboridia apicalis (Nawa); Arboridia kermanshah Dlabola; Arboridia parvula (Boheman); Arboridia velata (Ribaut); Arboridia sp. A (Japan); Arboridia sp. B (Lien Hua Chih, Taiwan); Arboridia sp. C (Meifeng, Taiwan); Arboridia sp. D (Tunshih, Taiwan); Cerneura sp. (Pakhuis Pass, South Africa); Chujophila sp. (Madagascar); Coloana arcuata Dworakowska; Diomma pulchra (Matsumura); Diomma (Bunyipia) sp. (Papua New Guinea); Frutioidia sp. (Madagascar); Helionidia dlabolai Dworakowska; N. Gen. A. (Papua New Guinea); N. Gen. B. (Islamabad, Pakistan); Gambialoa asiatica Dworakowska; Gambialoa gambiensis (Ross); Mitjaevia amseli (Dlabola); Molopopterus sp. (Madagascar); Musbrnoia sp. (N. Sumatra, Indonesia); Ratburella sp. (Taiwan); Salka nigricans (Matsumura); Seriana ochrata Dworakowska; Tautoneura sp. (N. Sumatra, Indonesia); Tautoneura mori (Matsumura); Thaia subrufa (Motschulsky); Ziczacella heptapotamica (Kuznetsova); Zygina flammigera (Geoffroy); Zyginidia scutellaris (Herrich-Schaeffer);

Ingroup New World Taxa. Amazygina compressa, n. sp.; Amazygina depressa, n. sp.; Amazygina decaspina, n. sp.; Aztegina punctinota, n. sp.; Erasmoneura nigerrima (McAtee); Erasmoneura vulnerata (Fitch); Eratoneura bifida (Beamer); Eratoneura impar (Beamer); Eratoneura ligata (McAtee); Eratoneura maculata (Gillette); Eratoneura osborni (DeLong); Erythridula aesculella (Ross and DeLong); Erythridula aspera (Beamer and Griffith); Erythridula atrimucronata Beamer; Erythridula crevcoeuri (Gillette); Erythridula obliqua (Say); Erythroneura calycula McAtee; Erythroneura comes (Say); Erythroneura corni Robinson; Erythroneura kanwakae Robinson; Hamagina pascoensis, n. sp.; Hamagina spinigera, n. sp.; Hepzygina aprica (McAtee); Hepzygina milleri (Beamer); Hymetta balteata McAtee; Hymetta trifasciata (Say); Illinigina illinoiensis (Gillette); Mexigina oculata (McAtee); Napogina recta, n. sp.; Napogina sinuata, n. sp.; Nelionidia amicis (Ross); Nelionidia elliptica, n. sp.; Neoimbecilla kiperi (Beamer); Neoimbecilla latiplata, n. sp.; Neozygina ceonothana (Beamer); Neozygina quadricornis (Beamer); Perugina denticula, n. sp.; Spinigina hirsuta, n. sp.; Spinigina quadrispinosa, n. sp.; Zyginama canyonensis (Beamer); Zyginama iguala (Ross); Zyginama ritana (Beamer 
APPENDIX C Data Matrix for Phylogenetic Analysis of Erythroneurini

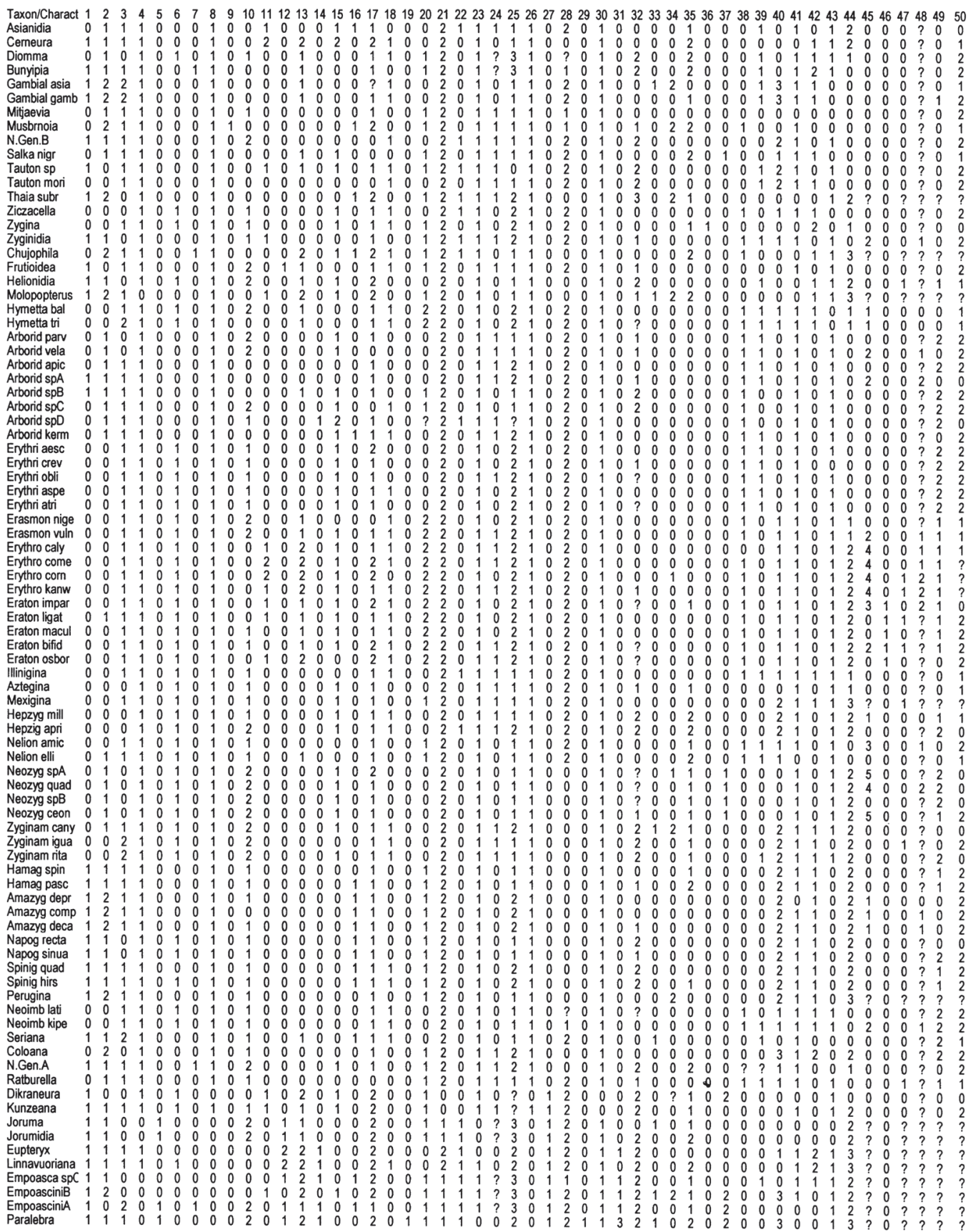




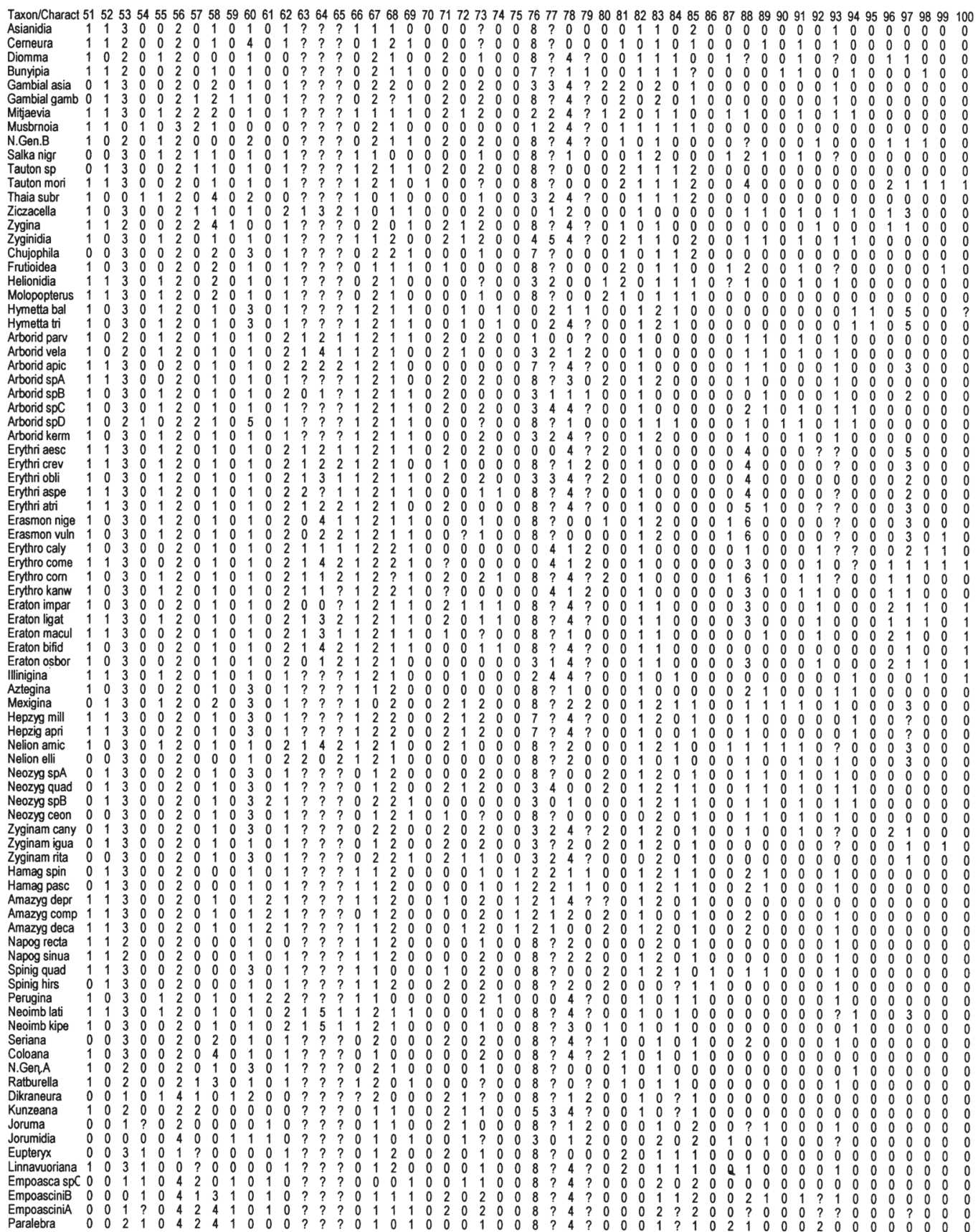


APPENDIX D Apomorphies for terminal taxa and internal nodes of cladogram (Fig. 12) based on ACCTRAN (accelerated transformation) optimization. Non-homoplastic changes are indicated by *.

Node Apomorphies

\begin{tabular}{|c|c|}
\hline 87 & $2(2), 20(1), 44(3), 91(0), 93(0)$ \\
\hline 88 & $13(2), 35(2), 39(0), 69(1), 81(1)$ \\
\hline 89 & $15(1), 17(2), 41(0), 43(1), 73(1), 91(1)$ \\
\hline 90 & $2(2), 40(3), 83(2)$ \\
\hline 91 & $3(2), 28(2), 33(1), 44(0), 51(0), 71(2), 77(3), 78(4), 84(0)$ \\
\hline 92 & $13(1), 32(0), 40(0), 50(1), 58(2), 66(0), 82(0)$ \\
\hline 93 & $35(1), 49(1), 51(0), 73(1), 79(1), 88(2), 89(1)$ \\
\hline 94 & $78(0), 88(2)$ \\
\hline 95 & $25(2), 77(1), 80(2), 84(0)$ \\
\hline 96 & $2(2), 45(1), 58(1), 61(2), 77(0), 78(4)$ \\
\hline 97 & $32(0), 75(1), 76(2), 78(1)$ \\
\hline 98 & $3(0), 6(1), 53(2), 84(0)$ \\
\hline 99 & $18(0)$ \\
\hline 100 & $25(2), 49(1), 71(1), 80(2), 86(1)^{*}$ \\
\hline 101 & 10(1), 16(1), 20(1), 39(0), 67(1), 78(2), 81(0), 83(2) \\
\hline 102 & 2(1), 24(0), 28(0), 44(2), 58(0), 68(2), 85(1), 93(1) \\
\hline 103 & $13(0), 20(0), 42(1), 43(0), 69(0), 71(0)$ \\
\hline 104 & 10(0), 39(1), 52(1), 66(1), 82(1), 85(2) \\
\hline 105 & $2(0), 13(1), 53(3), 78(0), 81(2), 84(1)$ \\
\hline 106 & $18(1), 40(2), 71(2)$ \\
\hline 107 & 13(1), 25(3), 28(1), 39(1), 42(1), 79(1), 91(1) \\
\hline 108 & 2(2), 16(1), 32(1), 34(2), 50(1), 53(0), 54(1), 76(1) \\
\hline 109 & $\begin{array}{l}\text { 6(1), 11(1), 15(1), 21(0)*, 30(0)*, 35(1), 71(2), 72(1), 77(3), 79(2), } \\
83(0)\end{array}$ \\
\hline 110 & 23(0), 31(0), 37(0), 43(0), 57(0), 72(1), 73(0), 78(1) \\
\hline 111 & $5(1), 10(2), 13(1), 41(0), 54(0)$ \\
\hline 112 & 14(0), 37(2), 71(2), 73(2), 89(1), 93(1) \\
\hline 113 & $3(0), 25(3), 31(1), 35(1), 44(2), 53(1), 61(1), 79(2), 83(2), 85(2)$ \\
\hline 114 & $\begin{array}{l}5(0), 10(0), 19(0)^{*}, 23(1)^{*}, 29(0)^{*}, 31(0), 33(0), 37(0), 40(0), 41(1), \\
58(0), 68(1), 87(0), 88(0), 92(0)\end{array}$ \\
\hline 115 & $12(2), 20(0), 42(2), 53(3), 62(1), 68(2), 81(2)$ \\
\hline 116 & $4(1), 12(0), 21(2)^{*}, 51(1), 56(0), 59(0), 69(0), 77(2), 84(1)$ \\
\hline 117 & $8(1), 14(0), 22(0), 24(1), 26(1), 44(0), 54(0), 56(2), 67(2), 82(1)$ \\
\hline 118 & $13(0), 27(0)^{*}, 28(0), 57(0), 58(1)$ \\
\hline 119 & $17(1), 60(1), 85(0)$ \\
\hline 120 & $10(2), 62(1), 81(1), 82(0), 84(0)$ \\
\hline 121 & 25(1), 28(2), 35(0), 69(1), 73(2) \\
\hline 122 & $2(0), 25(2), 42(2), 43(0), 44(1), 58(4)$ \\
\hline 123 & $1(0), 10(1), 32(0), 68(0)$ \\
\hline 124 & $38(1), 66(1), 84(1), 93(1)$ \\
\hline 125 & $68(1), 81(0), 82(1), 88(1), 91(1), 94(1)$ \\
\hline 126 & $67(1), 71(2), 72(1), 76(2), 81(2)$ \\
\hline 127 & $53(3), 55(1), 84(0)$ \\
\hline 128 & $3(0), 20(0), 39(1), 53(2), 79(2), 88(1)$ \\
\hline 129 & $24(0), 32(2), 63(0), 64(1), 77(1)$ \\
\hline 130 & $10(2), 32(1), 49(2), 62(2), 71(2), 76(3), 79(1), 83(0)$ \\
\hline 131 & $17(0), 78(1), 88(2), 89(1)$ \\
\hline 132 & $10(0), 15(1), 83(2), 94(0)$ \\
\hline
\end{tabular}


133

135

134

136

137

138

139

140

141

142

143

144

145

146

147

148

149

150

151

152

153

154

155

156

157

158

159

160

161

162

163

164

165

166

167

168

169

170

Amazyg comp

Amazyg depr

Amazyg deca

Asianidia

Arborid apic

Arborid kerm

Arborid parv

Arborid vela

Arborid spA

Arborid spB

Arborid spC

Arborid spD

Aztegina

Bunyipia

Cerneura

Chujophila
20(0), 25(2), 39(1), 65(2)

40(1), 49(0), 52(0), 64(0), 77(1), 78(2), 91(1)

15(0), 20(1), 25(1), 35(1), 84(1)

10(0), 13(1), 20(2), 25(2), 43(0), 45(1), 60(3), 69(1), 74(1), 77(2), $83(2), 95(1) *, 97(5)$

44(1), 48(0), 49(0), 50(1), 62(1), 72(1), 73(0), 76(0), 79(1)

39(1), 69(0), 97(0)

15(0), 25(1), 42(1), 64(5), 84(1), 94(1)

10(2), 50(1), 63(0), 78(0), 83(2), 87(1), 88(6)

48(1), 99(1)

47(1), 55(1), 71(2), 91(1), 98(0)

45(4), 46(0), 50(1), 68(2), 78(1), 83(0), 96(1), $100(0)$

13(2), 64(1), 73(0), 74(0), 76(0)

24(0), 71(2)

10(0), 11(1), 88(3), 98(1)

17(2), 24(1), 65(2), 74(1), 79(2)

44(2), 46(1), 48(2), 55(0), 64(3), 92(1), 96(2), 97(1), 100 (1)

13(1), 20(2), 44(1), 49(1), 83(1)

39(0), 40(1), 52(0), 77(4), 88(0)

18(0), 71(2), 73(2), 76(0), 80(2)

$97(2)$

18(1), 65(1), 69(1), 73(1)

$89(0)$

55(1), 88(4), 93(0)

$2(0), 6(1), 10(1), 24(0)$

49(2), 62(2), 73(0), 83(0), 89(1), 97(3)

52(1), 55(0), 69(0), 77(0), 91(0)

$32(1), 48(2), 50(0), 78(0)$

35(2), 42(0), 51(1), 76(7), 94(1)

49(0), 72(0), 80(2)

$3(2), 28(0), 66(0), 88(0)$

18(1), 32(2), 67(2), 76(3), 78(4), 89(0), 91(0), 93(0)

32(0), 40(2), 42(1), 48(0), 67(0), 71(2), 72(1), 77(2), 78(2), 97(1)

$45(5)$

76(3), 84(1), 94(1)

2(1), 24(0), 28(0), 37(1), 66(0)

10(2), 49(2), 51(0), 85(1), 91(1)

2(0), 3(0), 6(1), 10(1), 39(0), 60(3), 68(2), 89(1)

25(1), 35(1), 38(0), 44(2), 67(1)

$10(0), 66(0), 78(2), 83(0)$

41(0), 48(0), 71(1), 93(0)

$32(1), 72(1)$

11(1), 16(1), 22(1), 32(0), 83(1), 85(2), 88(0)

15(0), 63(2), 76(7)

16(1), 18(1), 76(3)

17(1), 76(1), 77(0), 78(0)

45(2), 49(0), 64(4), 69(0), 72(1), 73(0)

1(1), 15(0), 45(2), 71(2), 78(3), 80(2)

1(1), 10(0), 13(1), 17(1), 73(0), 88(0), 91(0), 97(2)

18(1), 62(1), 77(4), 78(4), 94(1)

14(1), 15(2), 22(1), 49(2), 50(0), 54(1), 57(2), 60(5), 78(1), 90(1)

44(1), 50(1), 52(0), 73(0), 78(1), 83(0), 88(2), 94(1)

7(1), 42(2), 52(1), 69(1), 73(0), 76(7), 78(1), 90(1), 94(1), 98(1)

11(2), 15(2), 53(2), 58(1), 60(4), 67(1), 84(0), 89(1)

1(0), 7(1), 16(1), 22(1), 38(1), 51(0), 52(0), 60(3), 76(7), 85(2) 


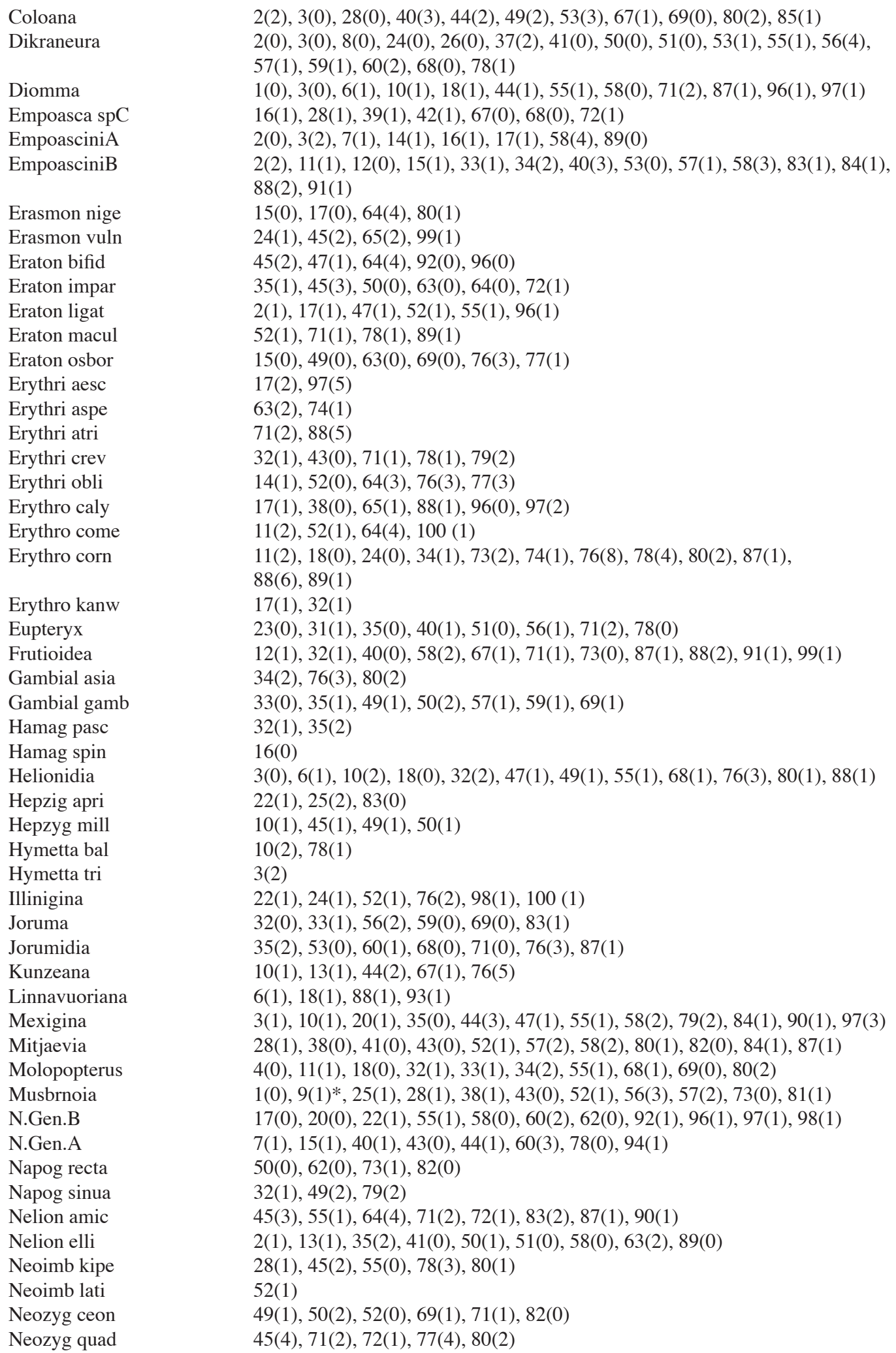


Neozyg spA

Neozyg spB

Perugina

Ratburella

Salka nigr

Seriana

Spinig hirs

Spinig quad

Tauton mori

Tauton sp

Thaia subr

Ziczacella

Zygina

Zyginam cany

Zyginam igua

Zyginam rita

Zyginidia
17(2), 34(1), 80(2)

61(2), 67(2), 69(1), 73(0), 78(1)

16(0), 34(2), 44(3), 52(0), 55(1), 62(2), 68(0), 74(1), 75(0), 76(0), 83(0)

17(0), 32(1), 39(1), 40(1), 47(1), 49(1), 50(1), 57(1), 58(3), 78(0)

13(1), 35(2), 37(1), 38(0), 40(1), 42(1), 43(0), 50(1), 51(0), 57(1), 67(1), 68(0), 69(0), 73(1), 87(1)

10(1), 16(1), 42(0), 49(2), 52(0), 68(0), 80(1), 81(0), 88(2)

6(1), 35(1), 51(0), 71(2), 82(0), 83(0)

60(3), 68(0), 78(0), 85(0), 88(1), 89(1)

$1(0), 17(0), 70(1)^{*}, 88(4), 96(2), 97(1), 98(1), 99(1), 100(1)$

11(1), 15(1), 22(1), 25(0), 51(0), 57(1)

3(0), 22(1), 32(3), 35(1), 41(0), 44(2), 55(1), 58(4), 60(2), 66(1), 67(0), $76(3), 85(2)$

3(0), 18(1), 22(1), 39(0), 42(1), 43(0), 57(1), 64(3), 67(0), 69(1), 73(2), 76(0), 94(1), 96(1)

6(1), 15(1), 18(1), 24(0), 35(1), 36(1)*, 41(0), 50(0), 52(1), 57(2), 59(1), 60(0), $71(2)$

72(1), 92(1), 96(1), 97(1)

2(1), 3(1), 25(2), 33(1), 34(2), 88(1), 91(1), 96(2)

15(0), 43(0), 47(1), 50(2), 60(1), 67(1), 78(2), 99(1)

$9(1), 52(0), 69(1), 73(1), 82(0)$

1(1), 3(0), 11(1), 22(1), 25(2), 32(1), 39(1), 40(1), 45(2), 68(2), 69(0), 76(4), 77(5), 85(2), 89(1) 


\section{APPENDIX E Old World Species Previously Placed in the Genus Erythroneura}

\section{Species Placed in Other Genera}

Accacidia acaciae (Linnavuori, 1962a: 72) (Erythroneura)

Accacidia dactyla (Naudé, 1926a: 102) (Erythroneura)

Accacidia garhiensis (Ahmed, 1970b: 181) (Erythroneura)

Accacidia improvisa (Linnavuori, 1953d: 61) (Erythroneura)

Arboridia (Arboridia) adanae (Dlabola, 1957a: 66) (Erythroneura)

Arboridia (Arboridia) agrillacea (Anufriev, 1969b: 182) (Erythroneura)

Arboridia (Arboridia) alpestris (Ribaut, 1959b: 400) (Erythroneura)

Arboridia (Arboridia) brevis (Ribaut, 1931c: 407) (Erythroneura)

Arboridia (Arboridia) defecta (Dlabola, 1957b: 294) (Erythroneura)

Arboridia (Arboridia) erecta (Ribaut, 1931c: 402) (Erythroneura)

Arboridia (Arboridia) ithaburensis (Linnavuori, 1962a: 70) (Erythroneura)

Arboridia (Arboridia) kermanshah (Dlabola, 1963a: 310) (Erythroneura)

Arboridia (Arboridia) kratochvili (Lang, 1945c: 96) (Erythroneura)

Arboridia (Arboridia) loginovae (Emeljanov, 1964f: 19) (Erythroneura)

Arboridia (Arboridia) maculifrons (Vilbaste, 1968a: 107) (Erythroneura)

Arboridia (Arboridia) pusilla (Ribaut, 1936b: 74) (Erythroneura)

Arboridia (Arboridia) remmi (Vilbaste, 1968a: 103) (Erythroneura)

Arboridia (Arboridia) ribauti (Ossiannilsson, 1937a: 25) (Erythroneura)

Arboridia (Arboridia) silvarum (Vilbaste, 1968a: 104) (Erythroneura)

Arboridia (Arboridia) simillima (Wagner, 1939a: 201) (Erythroneura)

Arboridia (Arboridia) suputinkaensis (Vilbaste, 1968a: 109) (Erythroneura)

Arboridia (Arboridia) velata (Ribaut, 1952a: 451) (Erythroneura)

Arboridia (Arboridia) vitisuga (Dlabola, 1963a: 312) (Erythroneura)

Arboridia (Arborifera) samadi (Samad \& Ahmed, 1979b: 2) (Erythroneura)

Arboridia (Arborifera) vinealis (Ahmed, 1970a: 32) (Erythroneura)

Asianidia albula (Lindberg, 1961a: 73) (Erythroneura)

Asianidia allagopappi (Lindberg, 1954a: 248) (Erythroneura)

Asianidia alticola (Lindberg, 1954a: 264) (Erythroneura)

Asianidia apiculata (Horváth, 1909a: 295) (Erythroneura)

Asianidia asiatica (Kusnezov, 1932a: 156) (Erythroneura)

Asianidia atlantica (China, 1938c: 49) (Erythroneura)

Asianidia bystropogonis (Lindberg, 1954a: 249) (Erythroneura)

Asianidia canariensis (Metcalf, 1955a: 267) (Erythroneura)

Asianidia chinai (Lindberg, 1961a: 71) (Erythroneura)

Asianidia chrysanthemi (Lindberg, 1954a: 250) (Erythroneura)

Asianidia decolor (Lindberg, 1936a: 15) (Erythroneura)

Asianidia insulana (Lindberg, 1961a: 70) (Erythroneura)

Asianidia lactea Lindberg 1954a: 250) (Erythroneura)

Asianidia madeirensis (China, 1938c: 48) (Erythroneura)

Asianidia octofasciata (Lindberg, 1936a: 11) (Erythroneura)

Asianidia perspicillata (Horváth, 1909a: 295) (Erythroneura)

Asianidia rubropunctata (Lindberg, 1936a: 14) (Erythroneura)

Asianidia salviae (Lindberg, 1954a: 253) (Erythroneura)

Asianidia spartocytisi (Lindberg, 1936a: 13) (Erythroneura)

Asianidia sublactea (Lindberg, 1954a: 249) (Erythroneura)

Asianidia tejedae (Lindberg, 1954a: 252) (Erythroneura)

Asianidia tenerifae (Lindberg, 1936a: 13) (Erythroneura)

Asianidia vallicola (Lindberg, 1954a: 251) (Erythroneura)

Beamerana tropicalis (Osborn, 1928a: 288) (Erythroneura)

Cassianeura cassiae (Ahmed, 1970a: 34) (Erythroneura)

Ciudadrea colorata (Lindberg, 1936a: 12) (Erythroneura)

Ciudadrea magnifica (Lindberg, 1954a: 243) (Erythroneura)

Dikraneura (Dikraneura) mali (Provancher, 1890a: 297) (Erythroneura)

Empoasca carneola (Osborn, 1928a: 288) (Erythroneura)

Empoascanara (Empoascanara) alami (Ahmed, 1970b: 183) (Erythroneura) 
Empoascanara (Empoascanara) hazarensis (Ahmed, 1970b: 179) (Erythroneura) Empoascanara (Empoascanara) sathyamangalamensis (Sohi \& Kapoor, 1974a: 42) (Erythroneura) Empoascanara (Empoascanara) truncata (Ahmed, 1971b: 183) (Erythroneura) Frutioidia (Dworakowskellina) amicula (Linnavuori, 1965a: 16) (Erythroneura) Frutioidia (Frutioidia) ricei (Ross, 1965a: 265) (Erythroneura) Gambialoa gambiensis (Ross, 1965a: 265) (Erythroneura) Hauptidia (Hauptidia) armata (Ribaut, 1948a: 4) (Erythroneura) Hauptidia (Hauptidia) provincialis (Ribaut, 1931c: 414) (Erythroneura) Hauptidia (Hauptidia) rivularis (Linnavuori, 1962a: 72) (Erythroneura) Hauptidia (Hauptidia) soosi (Dlabola, 1952b: 31) (Erythroneura) Hauptidia (Hauptidia) stellata (Ribaut, 1948a: 5) (Erythroneura) Helionidia cicadulosa (Naudé, 1926a: 101) (Erythroneura) Helionidia longifalx (Linnavuori, 1953d: 62) (Erythroneura) Helionidia quadrimaculata (Naudé, 1926a: 100) (Erythroneura) Henribautia hubbardi (McAtee, 1924c: 35) (Erythroneura) Imbecilla imbecilla (Linnavuori, 1962a: 72) (Erythroneura) Imbecilla lubiae (China, 1931a: 53) (Erythroneura) Kapsa mingorensis (Ahmed, 1970a: 40) (Erythroneura) Kropka unipunctata (Dlabola, 1957c: 119) (Erythroneura) Kusala salicis (Ahmed, 1970a: 39) (Erythroneura) Kusala setosa (Ahmed, 1971b: 181) (Erythroneura) Lamtoana flavoscuta (Naudé, 1926a: 102) (Erythroneura) Lectotypella kabiri (Ahmed, 1971b: 179) (Erythroneura) Lublinia gediensis (Linnavuori, 1962a: 72) (Erythroneura) Melicharidia aridula (Linnavuori, 1956b: 138) (Erythroneura) Mitjaevia amseli (Dlabola, 1961b: 297) (Erythroneura) Mitjaevia aurantiaca (Mitjaev, 1969b: 1045) (Erythroneura) Mitjaevia bibichanae (Dlabola, 1961b: 296) (Erythroneura) Molopopterus pulchra (Naudé, 1926a: 103) (Erythroneura) Molopopterus steeleae (Ross, 1965a: 66) (Erythroneura) Ossiannilssonola australis (Walsh, 1862a: 149) (Erythroneura) Protalebra sublunata (Osborn, 1928a: 289) (Erythroneura) Punctigerella betulae (Vilbaste, 1968a: 112) (Erythroneura) Punctigerella juchani (Anufriev, 1971e: 520) (Erythroneura) Punctigerella koreana (Anufriev, 1971e: 521) (Erythroneura) Punctigerella lamellaris (Vilbaste, 1968a: 111) (Erythroneura) Qadria cajanae (Ahmed, 1971b: 185) (Erythroneura) Rhusia maculicosta (Naudé, 1926a: 99) (Erythroneura) Sempia capreola (Linnavuori, 1964a: 340) (Erythroneura) Singapora cyclops (Kusnezov, 1932a: 155) (Erythroneura) Tamaricella atriplicis (Lindberg, 1954a: 256) (Erythroneura) Tamaricella cypria (Ribaut, 1948a: 6) (Erythroneura) Tamaricella franckeniae (Lindberg, 1954a: 254) (Erythroneura) Tamaricella fuerteventurae (Lindberg, 1954a: 255) (Erythroneura) Tamaricella tangigharuha (Dlabola, 1957b: 295) (Erythroneura) Tautoneura albida (Dworakowska, 1970d: 353) (Erythroneura) Tautoneura deska (Dworakowska, 1970d: 348) (Erythroneura) Tautoneura formosa (Dworakowska, 1970d: 353) (Erythroneura) Tautoneura fusca (Dworakowska, 1970d: 353) (Erythroneura) Tautoneura indefinita (Dworakowska, 1970d: 348) (Erythroneura) Tautoneura japonica (Dworakowska, 1972g: 865) (Erythroneura) Tautoneura leucothoe (Kirkaldy, 1907d: 70) (Erythroneura) Tautoneura marthae (Linnavuori, 1960a: 268) (Erythroneura) Tautoneura sinica (Dworakowska, 1970d: 348) (Erythroneura) Ziczacella dworakowskae (Anufriev, 1969c: 698) (Erythroneura) Ziczacella heptapotamica (Kusnezov, 1928b: 316) (Erythroneura) Ziczacella lyrifora (Dlabola, 1968a: 368) (Erythroneura) Ziczacella steggerdai (Ross, 1965a: 267) (Erythroneura) Zygina (Hypericiella) digitata (Ribaut, 1948a: 3) (Erythroneura) 
Zygina (Hypericiella) nebulosa (Ribaut, 1948a: 3) (Erythroneura) Zygina (Zygina) alnicola (Linnavuori, 1965a: 35) (Erythroneura) Zygina (Zygina) dorycnii (Ribaut, 1936b: 52) (Erythroneura) Zygina (Zygina) karatasa (Dlabola, 1957a: 66) (Erythroneura) Zygina (Zygina) ordinaria (Ribaut, 1936b: 47) (Erythroneura) Zygina (Zygina) rosincola (Cerutti, 1939a: 84) (Erythroneura) Zygina (Zygina) schneideri (Günthart, 1974a: 24) (Erythroneura) Zygina (Zygina) ulmi (Ahmed, 1970a: 33) (Erythroneura) Zygina (Zygina) ulmicola (Mitjaev, 1971a: 118) (Erythroneura) Zyginidia (Zyginidia) alexandrina (Linnavuori, 1964a: 340) (Erythroneura) Zyginidia (Zyginidia) alpicola (Cerutti, 1939a: 86) (Erythroneura) Zyginidia (Zyginidia) franzi (Wagner, 1944b: 38) (Erythroneura) Zyginidia (Zyginidia) lineata (Lindberg, 1954a: 244) (Erythroneura) Zyginidia (Zyginidia) mocsaryi (Horváth, 1910b: 176) (Erythroneura) Zyginidia (Zyginidia) younasi (Ahmed \& Samad, 1980) (Erythroneura) Zyginopsis verticalis (Ahmed, 1970a: 31) (Erythroneura)

\section{Species Incertae Sedis}

Erythroneura ariadne Linnavuori, 1960b: 22

Erythroneura atropictila Ahmed, 1970a: 35

Erythroneura cassavae China, 1930a: 267

Erythroneura chaudhrii Samad \& Ahmed, 1979b: 2

Erythroneura claripennis Naudé, 1926a: 98

Erythroneura doris Kirkaldy, 1907d: 69

Erythroneura evansi Ross, 1965a: 267

Erythroneura fulvidorsum Naudé, 1926a: 98

Erythroneura harmsi Izzard, 1936a: 600

Erythroneura honiala Kirkaldy, 1906c: 365

Erythroneura honiloa Kirkaldy, 1906c: 365

Erythroneura ipoloa Kirkaldy, 1906c: 365

Erythroneura kashmirensis Ahmed, 1970a: 37

Erythroneura lalage Kirkaldy, 1907d: 70

Erythroneura leveri Evans, 1948a: 131

Erythroneura lubra Kirkaldy, 1906c: 364

Erythroneura macarangae Linnavuori, 1960a: 266

Erythroneura melanogaster Kirkaldy, 1906a: 364

Erythroneura modesta Ahmed, 1970a: 41

Erythroneura nayavua Linnavuori, 1960b: 21

Erythroneura plagiata Heller \& Linnavuori, 1968a: 17

Erythroneura postica Heller \& Linnavuori, 1968a: 17

Erythroneura rewana Kirkaldy, 1907d: 71

Erythroneura sativae Evans, 1940c: 12

Erythroneura shirozui Ishihara, 1965

Erythroneura sidnica Kirkaldy, 1907d: 69

Erythroneura sohii Ahmed, 1985c: 251

Erythroneura subfumata Heller \& Linnavuori, 1968a: 16

Erythroneura toetoe Cumber, 1952a: 525

Erythroneura urakensis Samad \& Ahmed, 1979b: 3

Erythroneura zealandica Myers, 1923a: 424

\section{Nomina Nuda}

Erythroneura bistrig Esaki \& Ito, 1954a: 213

Erythroneura flavogutta Esaki \& Ito, 1954a: 215

Erythroneura hirtipennis Lindberg, 1948b: 162 

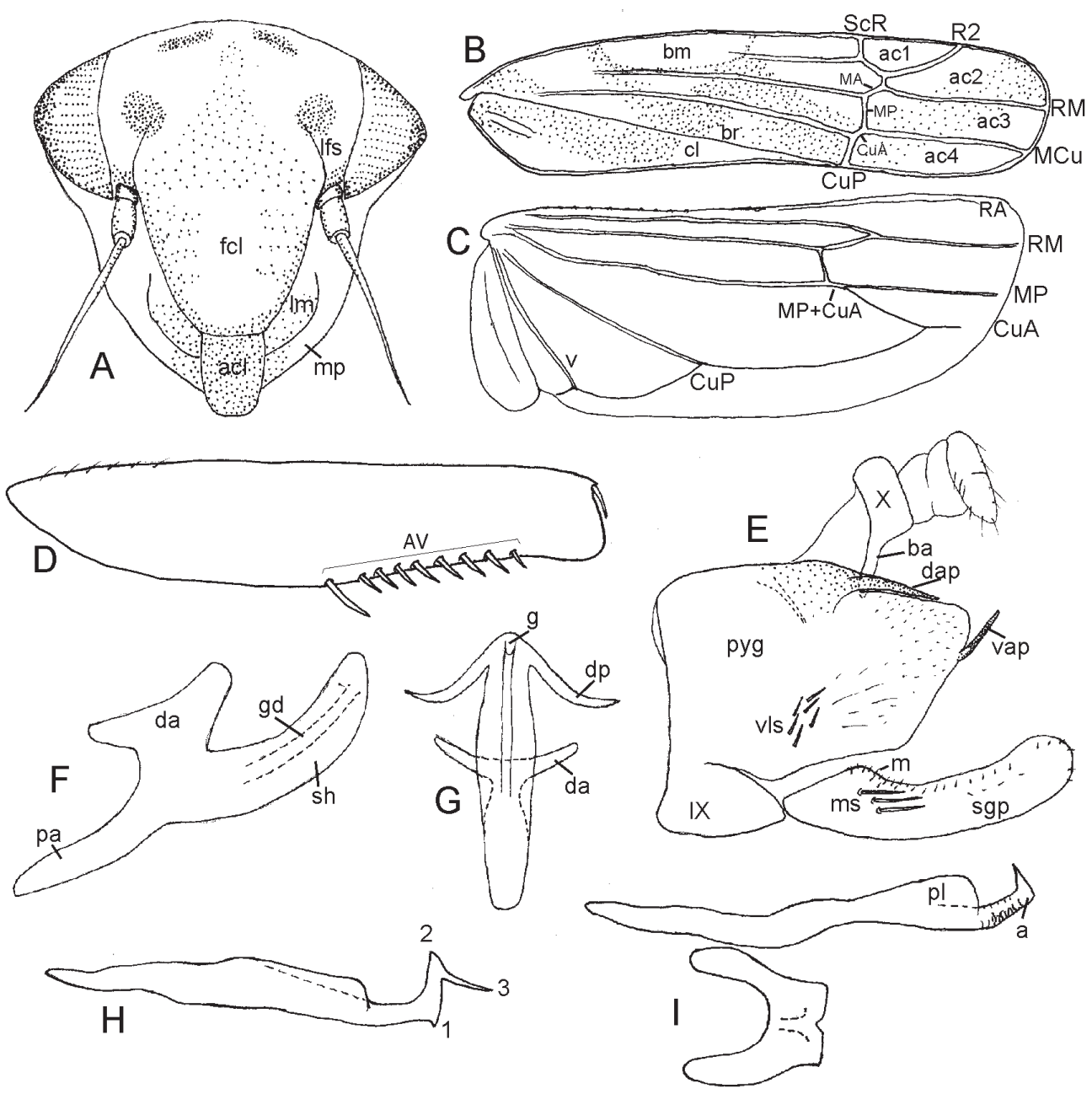

Figure 1. Erythroneurini morphology. A, Mexigina oculata (McAtee), head, anterior view; B-C, Erythroneura kanwakae Beamer, forewing (B) and hind wing (C); D, M. oculata, front femur, anterior view; E, Zyginama ritana (Beamer), male genital capsule, lateral view; F, Z. nuda (Knull and Auten), aedeagus, lateral view; G, Neozygina ceonothana (Beamer), aedeagus, posterior view; H, Nelionidia moyai, n. sp., male style, ventrolateral view, distal points numbered as shown; I, Amazygina decaspina, n. sp., left style and connective, ventral view. Abbreviations: a, apophysis; ac, apical cell; acl, anteclypeus; ba, basolateral arm; bm, brochosome field; br, brachial cell; cl, clavus; da, dorsal apodeme; dap, dorsal appendage; dp, distal process; fcl, frontoclypeus; g, gonopore; gd, gonoduct; lfs, lateral frontal suture; lm, lorum; m, marginal setae; mp, maxillary plate; ms, macrosetae; pa, preatrium; pl, preapical lobe; pyg, pygofer; sgp, subgenital plate; sh, shaft; v, vannal vein; vap, ventral appendage; vls, ventrolateral setae. 


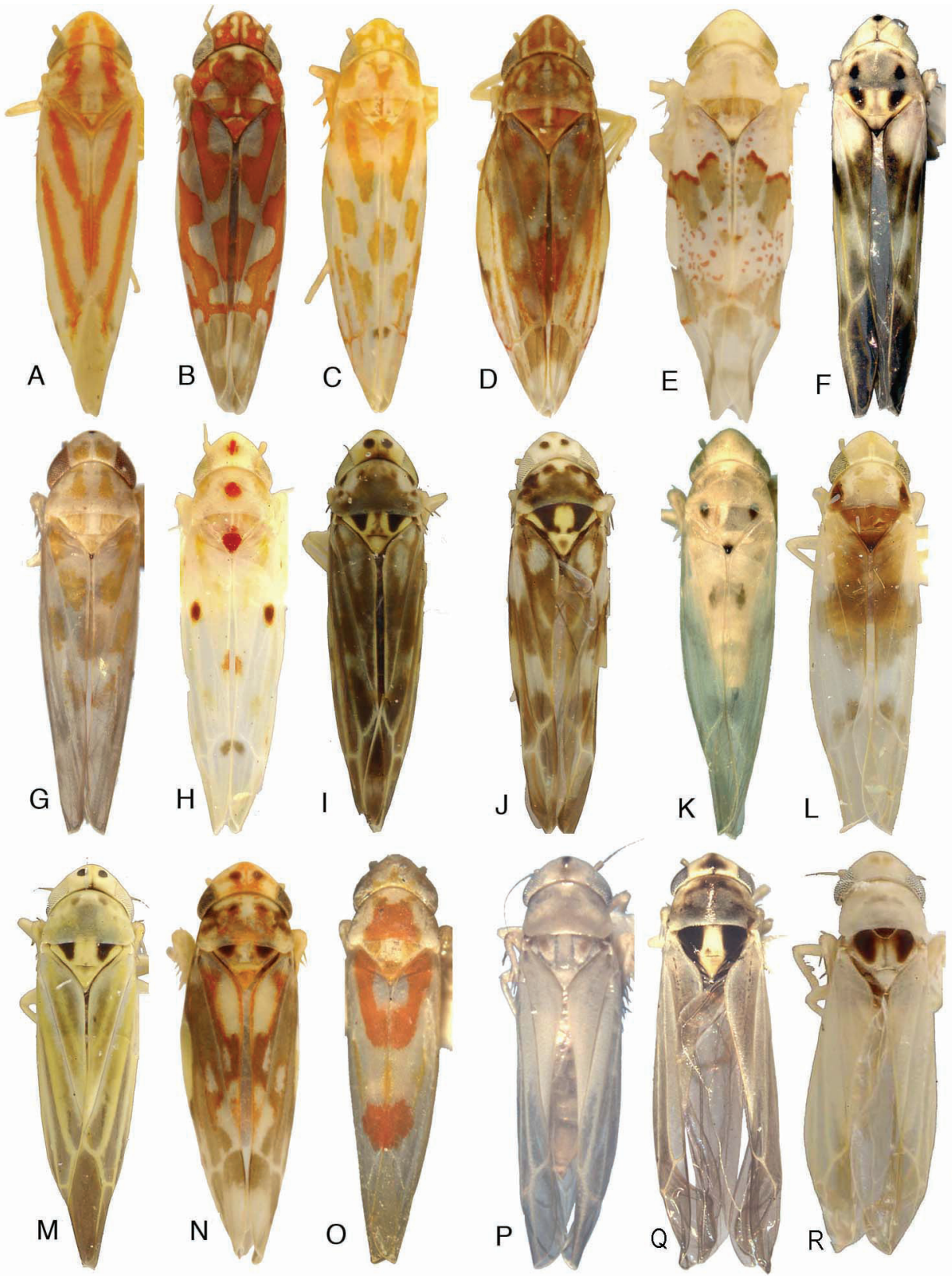

Figure 2. Dorsal habitus. A, Erythridula insigna (Beamer and Griffith); B, Erythroneura corni Robinson; C, Eratoneura dira (Beamer); D, Erasmoneura vulnerata (Fitch); E, Hymetta trifasciata (Say); F, Aztegina punctinota, n. sp.; G, Hepzygina milleri (Beamer); H, Illinigina illinoiensis (Gillette); I, Mexigina oculata (McAtee); J, Nelionidia elliptica n. sp.; K, Neoimbecilla kiperi (Beamer); L, Neoimbecilla latiplata, n. sp.; M, Neozygina apacha (Baker); N, Rossmoneura tecta (McAtee); O, Zyginama ritana; P, Amazygina compressa, n. sp.; Q, Hamagina spinigera, n. sp.; R, Napogina sinuata, n. sp. 


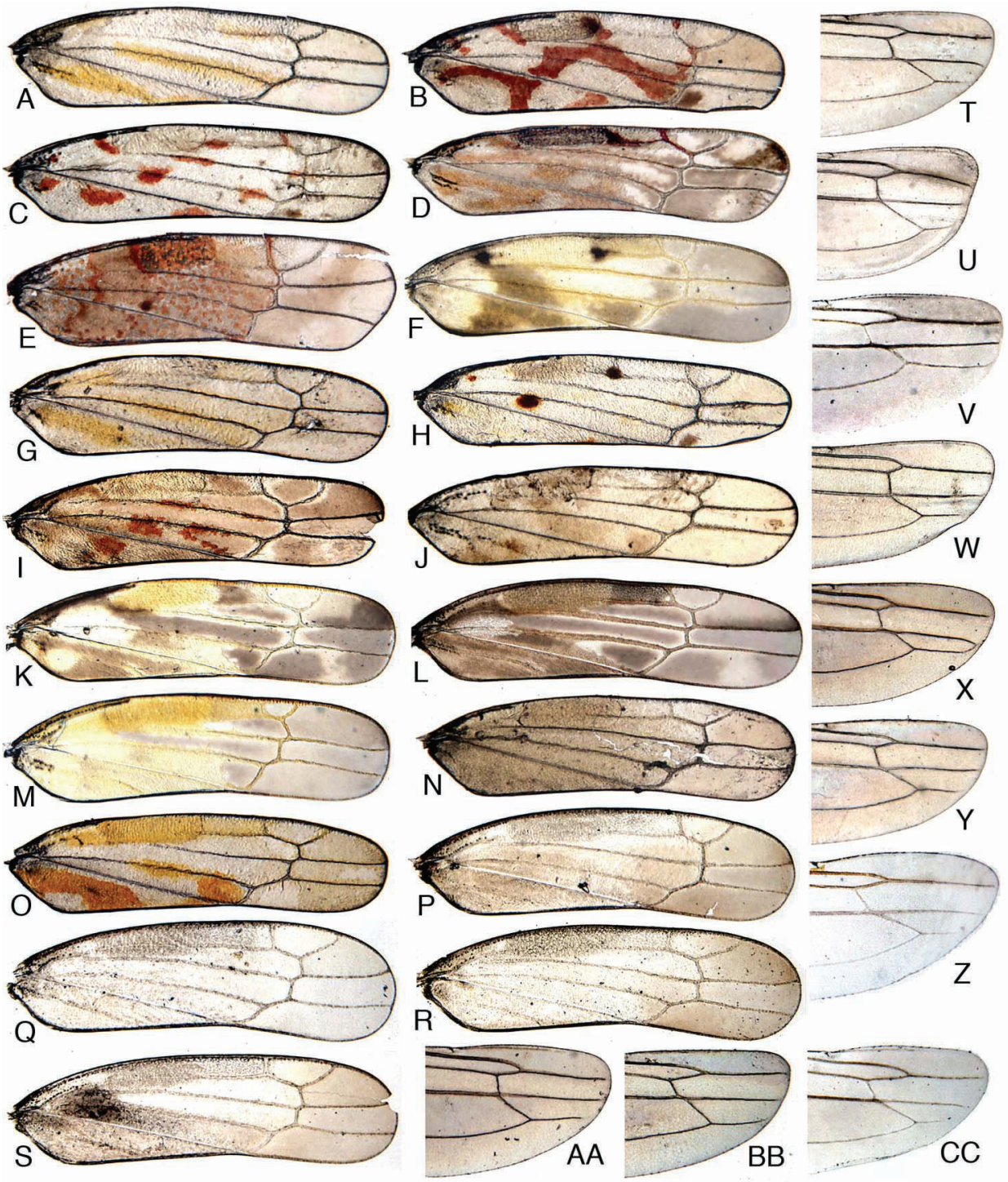

Figure 3. A-S, forewing: A, Erythridula obliqua (Say); B, Eratoneura ligata (McAtee); C, Eratoneura maculata (Gillette); D, Erasmoneura vulnerata (Fitch); E, Hymetta balteata McAtee; F, Aztegina punctinota, n. sp.; G, Hepzygina milleri (Beamer); H, Illinigina illinoiensis (Gillette); I, Mexigina oculata (McAtee); J, Neoimbecilla kiperi (Beamer); K, Nelionidia moyai, n. sp.; L, Nelionidia pueblensis, n. sp.; M, Nelionidia pallescens, n. sp.; N, Neozygina ceonothana (Beamer); $\mathrm{O}$, Zyginama ritana (Beamer); $\mathrm{P}$, Amazygina decaspina, n. sp.; Q, Hamagina spinigera, n. sp.; R, Perugina denticula, n. sp.; S, Spinigina hirsuta, n. sp. T-CC, hind wing: T, Erythridula obliqua; U, Erasmoneura vulnerata; V, Aztegina punctinota; W, Illinigina illinoiensis; X, Mexigina oculata (unusual specimen with closed apical cell); Y, Neoimbecilla kiperi; Z, Nelionidia pallescens; AA, Neozygina ceonothana; BB, Zyginama ritana; CC, Amazygina decaspina. 

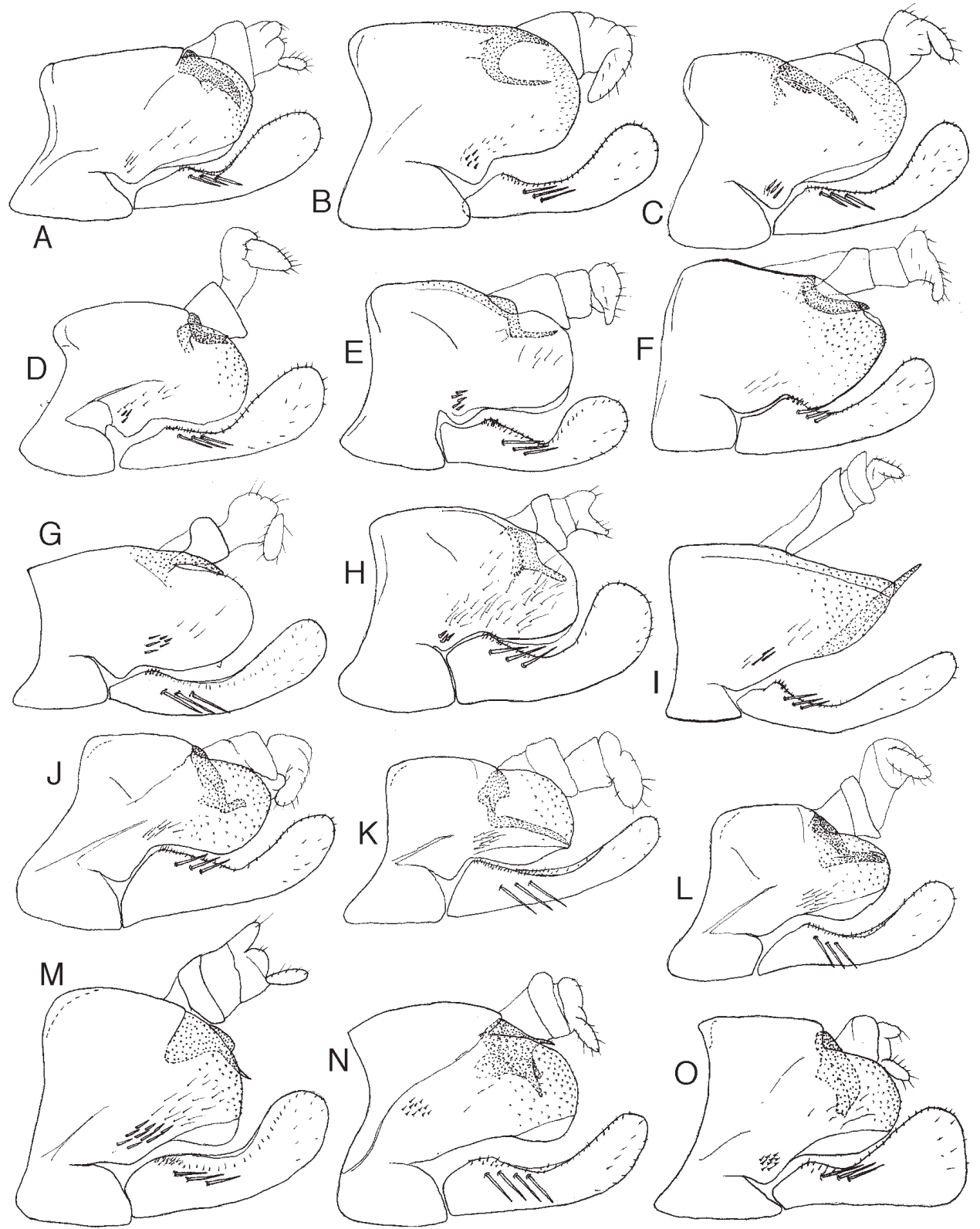

Figure 4. Male genital capsule, lateral view. A, Erythridula obliqua; B, Erythroneura comes (Say); C, Eratoneura maculata; D, Erasmoneura vulnerata; E, Hymetta balteata McAtee; F, Aztegina punctinota, n. sp.; G, Hepzigina milleri; H, Illinigina illinoiensis; I, Mexigina oculata; J, Nelionidia elliptica; K, Nelionidia moyai; L, Nelionidia pallescens; M, Nelionidia pueblensis; N, Neoimbecilla kiperi; O, Neoimbecilla latiplata. 

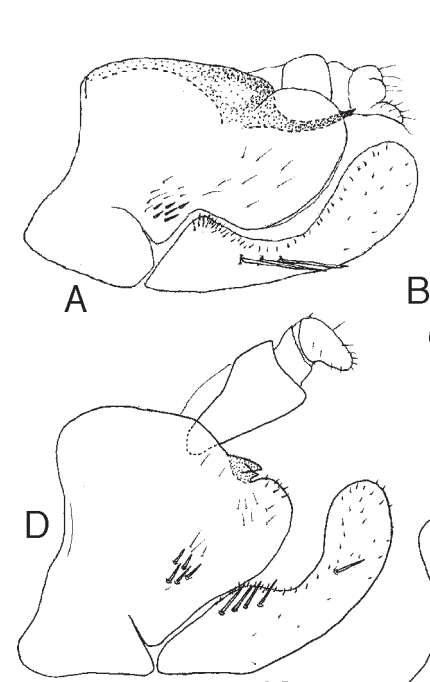

B
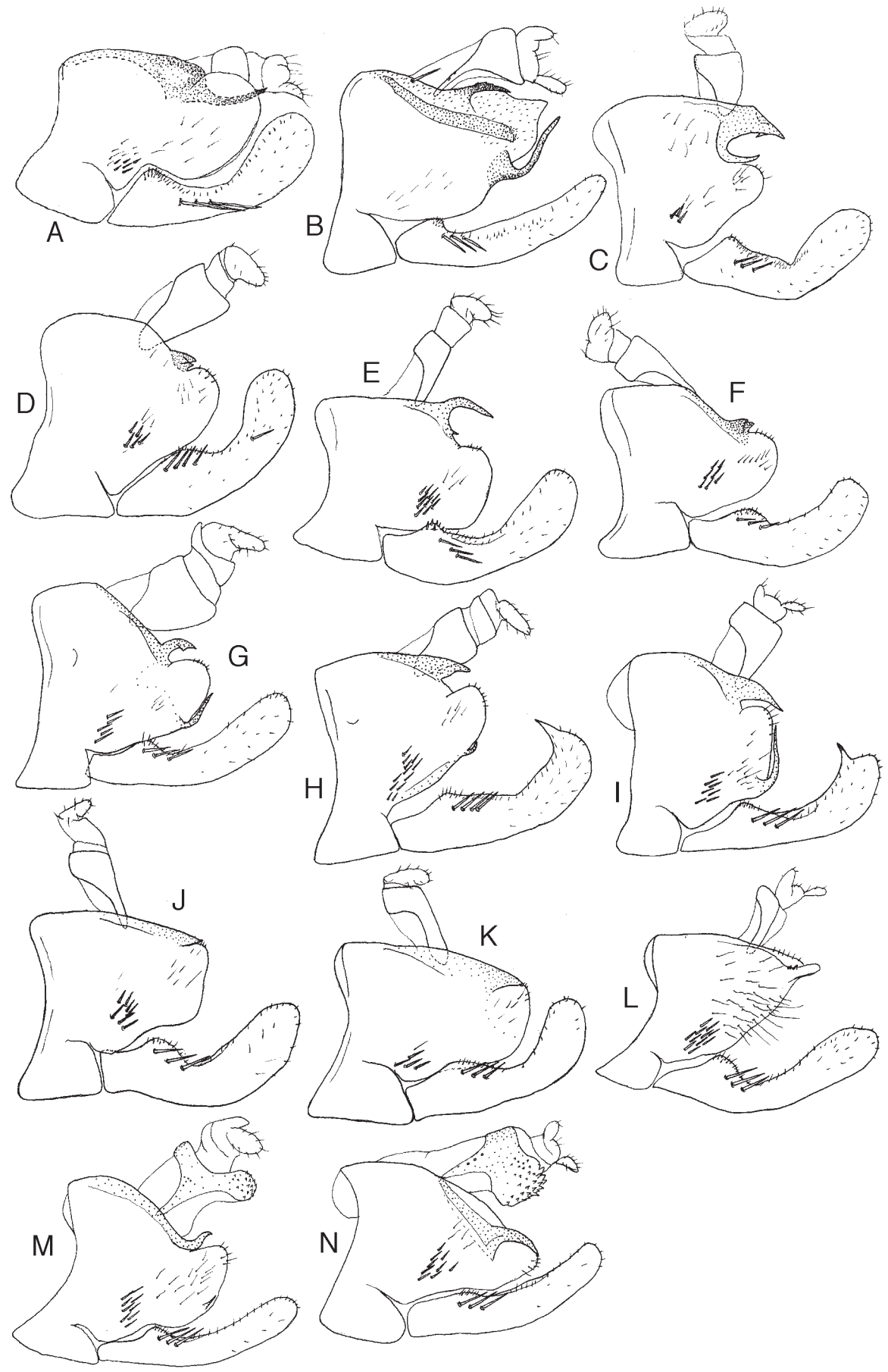

Figure 5. Male genital capsule, lateral view. A, Rossmoneura tecta; B, Neozygina ceonothana; C, Amazygina chela, n. sp.; D, Amazygina compressa, n. sp.; E, Amazygina depressa, n. sp.; F, Amazygina decaspina, n. sp.; G, Hamagina pascoensis, n. sp.; H, Hamagina serrata, n. sp.; I, Hamagina spinigera, n. sp.; J, Napogina recta, n. sp.; K, Napogina sinuata, n. sp.; L, Perugina denticula, n. sp.; M, Spinigina hirsuta, n. sp.; N, Spinigina quadrispinosa, n. sp. 


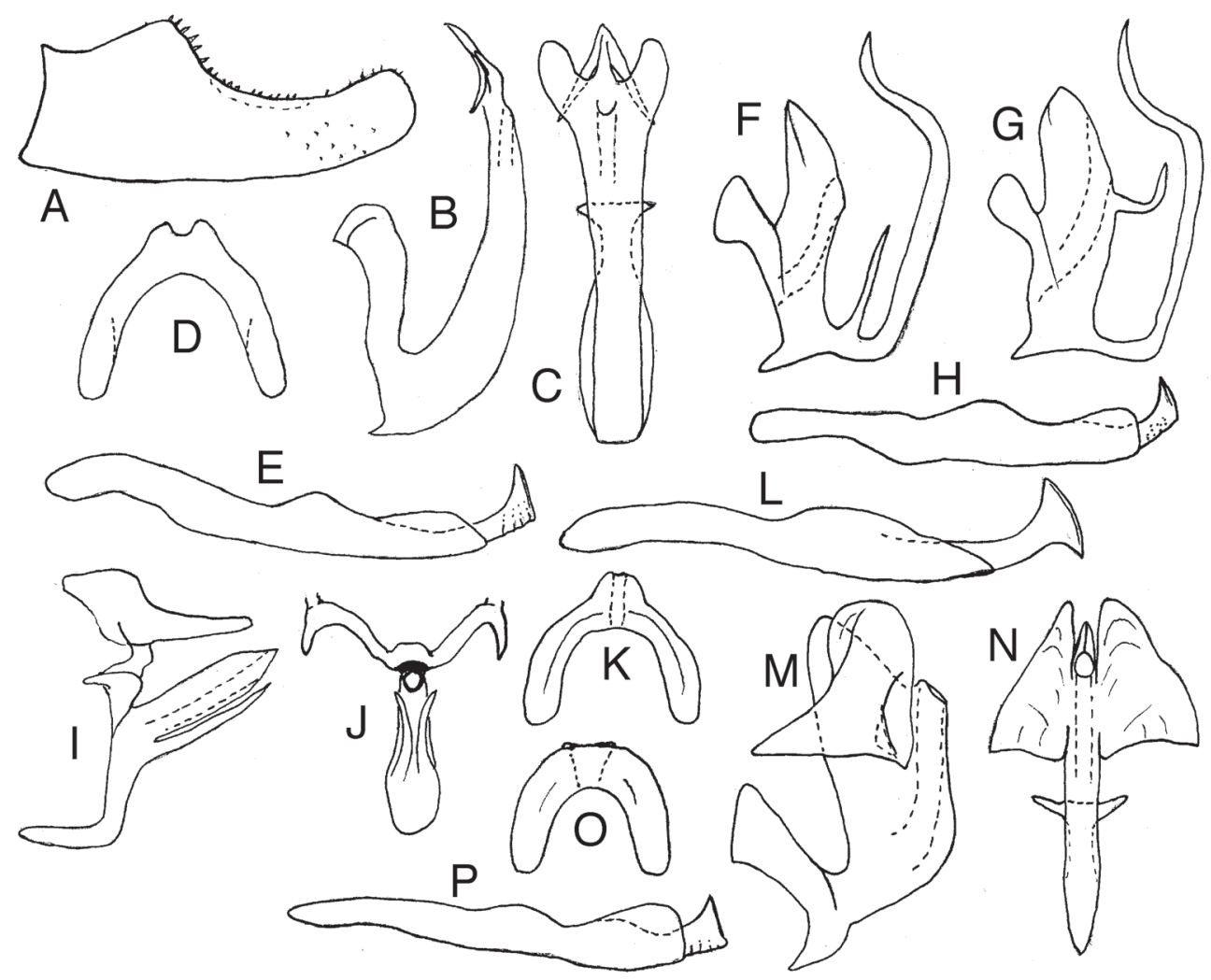

Figure 6. Male genitalia. A-D, Aztegina punctinota: A, subgenital plate, ventral view; B, aedeagus, lateral view; $\mathrm{C}$, same, posterior view; $\mathrm{D}$, connective, ventral view. $\mathrm{E}$, style, lateral view; F, Hepzygina milleri, aedeagus, lateral view. G-H, Hepzygina aprica: G, aedeagus, lateral view; $\mathrm{H}$, style, lateral view. I-L, Illinigina illinoiensis: I, aedeagus and dorsal pygofer appendage, lateral view; J, same, posterior view; K, connective, ventral view; L, style, lateral view. M-P, Mexigina oculata: $\mathrm{M}$, aedeagus, lateral view; $\mathrm{N}$, same, posterior view; O, connective, ventral view; $\mathrm{P}$, style, lateral view. 


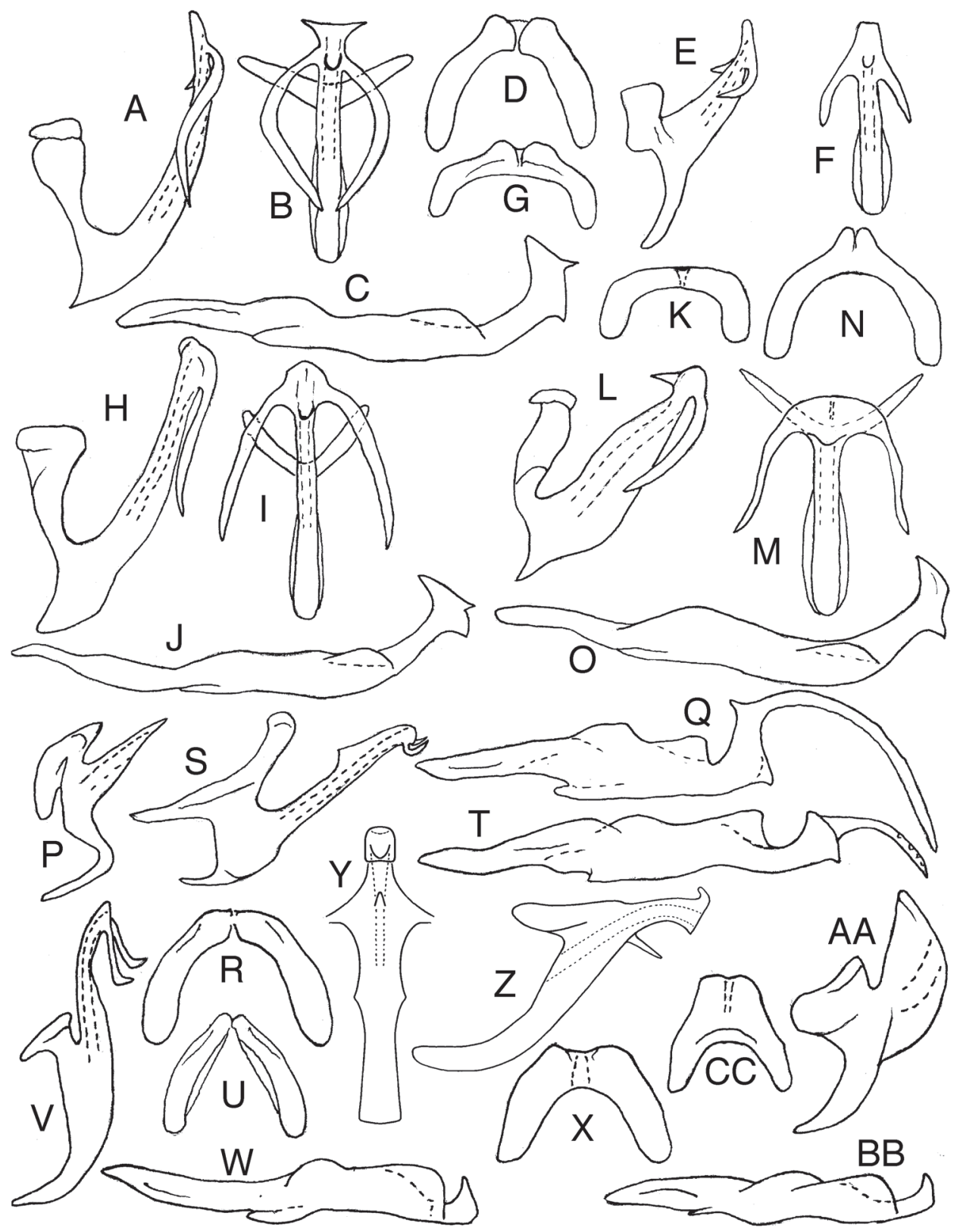

Figure 7. Male genitalia. A-C, Nelionidia elliptica: A, aedeagus, lateral view; B, same, posterior view; C, style, lateral view; D, connective, ventral view. E-G: Nelionidia moyai: E, aedeagus, lateral view; F, same, posterior view; G, connective, ventral view. H-K, Nelionidia pallescens: $\mathrm{H}$, aedeagus, lateral view; I, same, posterior view; J, style, lateral view; K, connective, ventral view. L-N, Nelionidia pueblensis: L, aedeagus, lateral view; M, same, posterior view; N, connective, ventral view; O, style, lateral view. P-R, Neoimbecilla latiplata: P, aedeagus, lateral view; Q, style ventral view; R, connective, ventral view. S-U, Neoimbecilla kiperi: S, aedeagus, lateral view; T, style, ventral view; U, connective, ventral view. V-X, Neozygina ceonothana: V, aedeagus, lateral view; W, style, ventrolateral view; X, connective, ventral view. Y-Z, Rossmoneura tecta, aedeagus, posteroventral and lateral views, respectively. AA-CC, Zyginama ritana: AA, aedeagus, lateral view; BB, style, ventral view; $\mathrm{CC}$, connective, ventral view. 


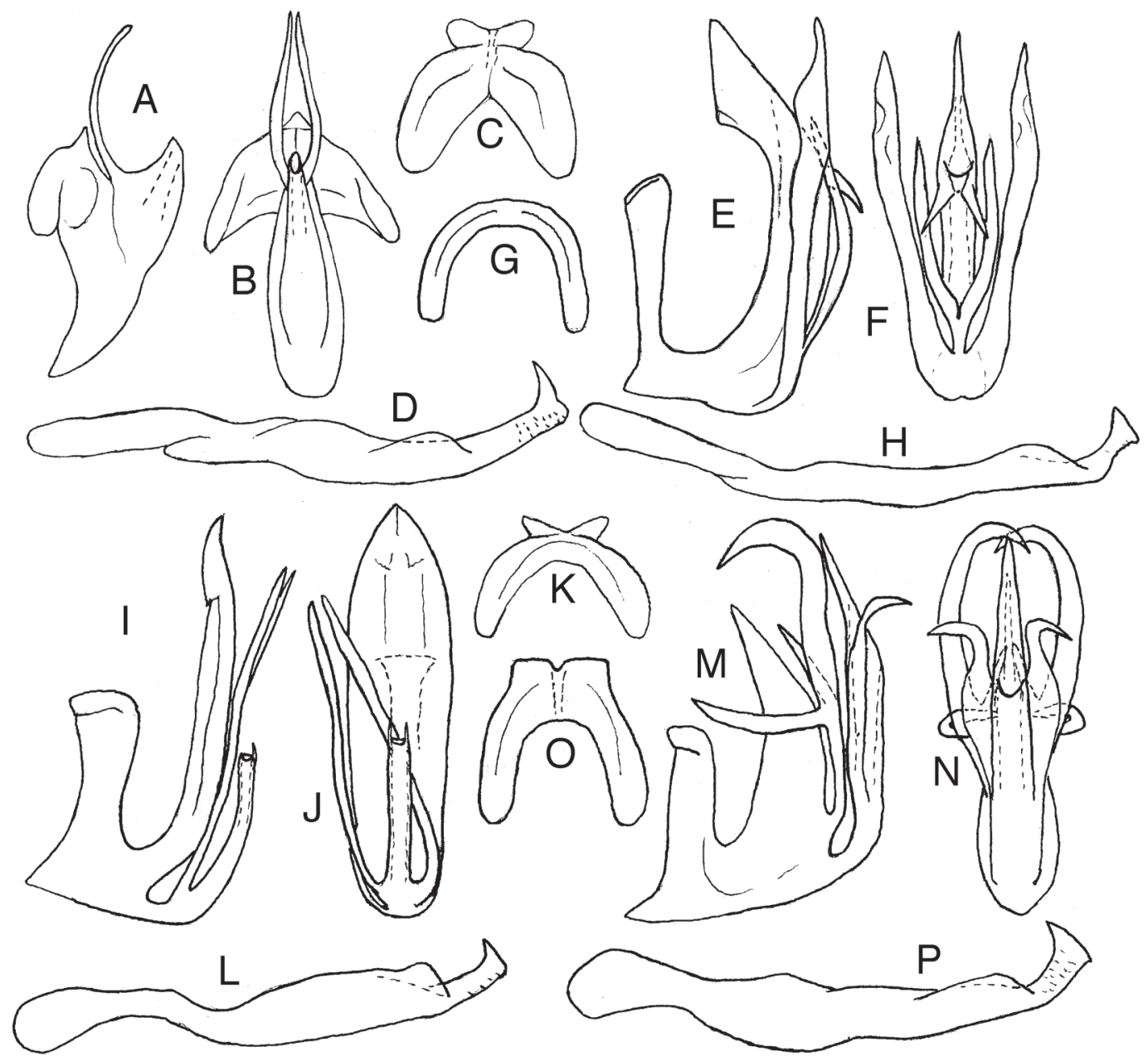

Figure 8. Amazygina, male genitalia. A-D, Amazygina chela: A, aedeagus, lateral view; B, same, posterior view; C, connective, ventral view; D, style, lateral view. E-H: Amazygina compressa: E, aedeagus, lateral view; F, same, posterior view; $\mathrm{G}$, connective, ventral view; $\mathrm{H}$, style, lateral view. I-L, Amazygina depressa: I, aedeagus, lateral view; J, same, posterior view; K, connective, ventral view; L, style, lateral view; M-P, Amazygina decaspina: M, aedeagus, ventral view; N, same, posterior view; O, connective, ventral view; P, style, lateral view. 


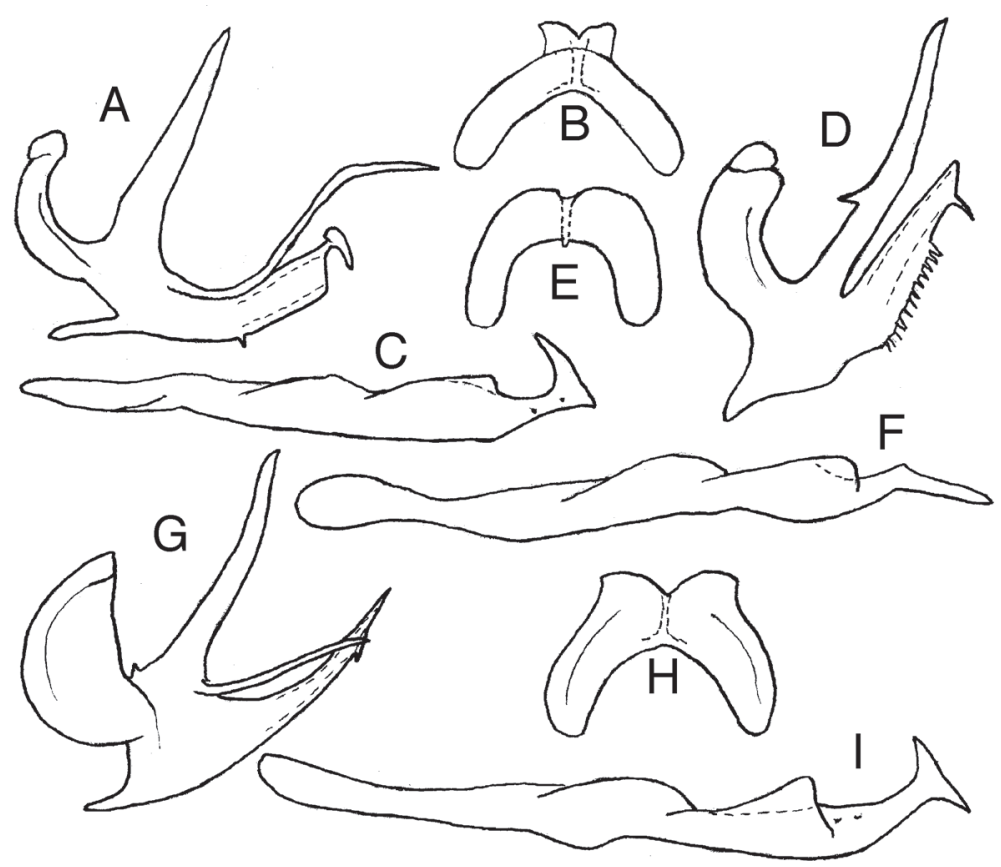

Figure 9. Hamagina, male genitalia. A-C, Hamagina pascoensis: A, aedeagus, lateral view; B, connective, ventral view; C, style, ventral view. D-F: Hamagina serrata: D, aedeagus, lateral view; E, connective, ventral view; F, style, ventrolateral view. G-I, Hamagina spinigera: G, aedeagus, lateral view; $\mathrm{H}$, connective, ventral view; I, style, ventral view. 


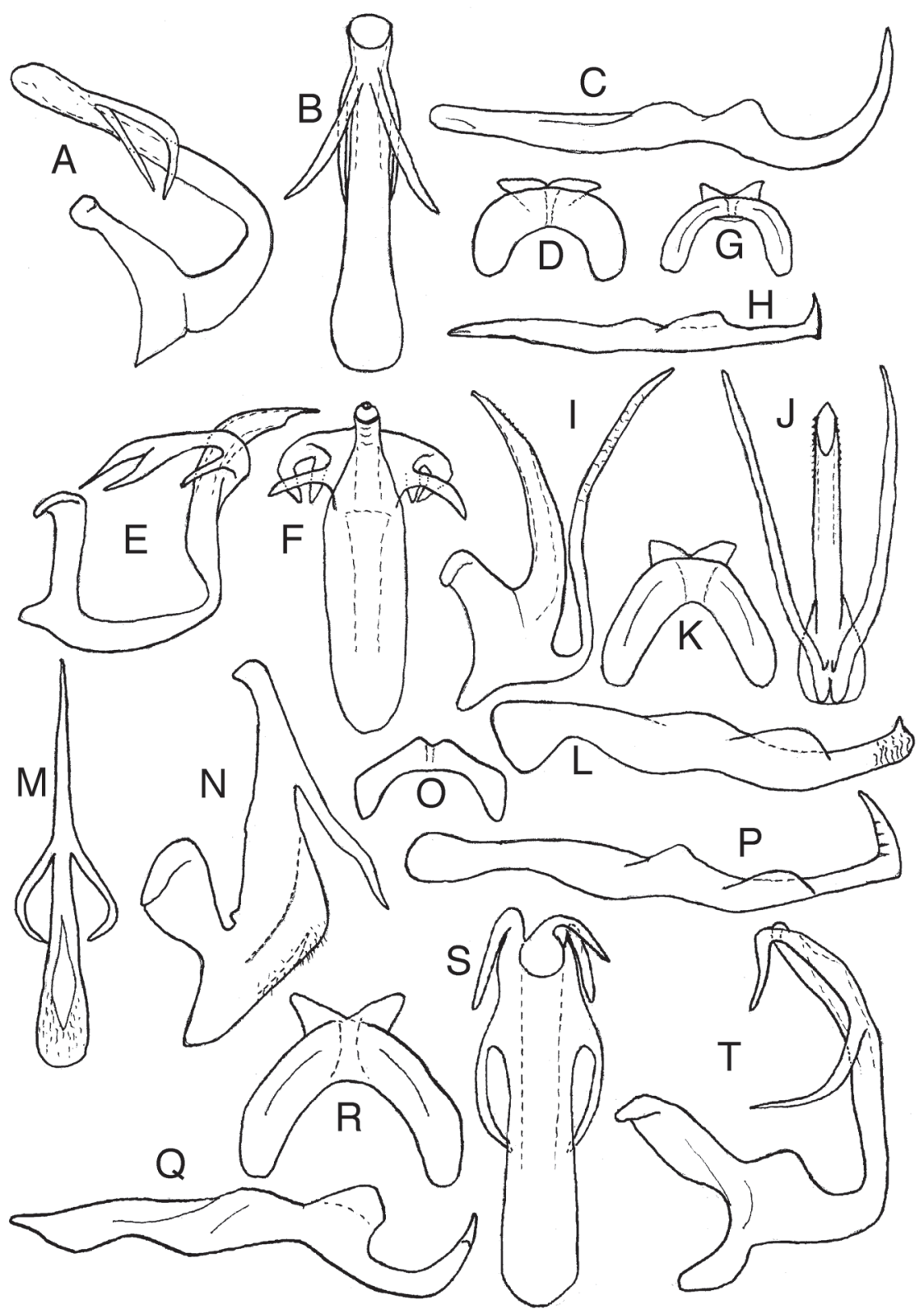

Figure 10. Male genitalia. A-D, Napogina recta: A, aedeagus, lateral view; B, same, posterior view; C, style, ventral view; D, connective, ventral view. E-H: Napogina sinuata: E, aedeagus, lateral view; F, same, posterior view; $\mathrm{G}$, connective, ventral view; $\mathrm{H}$, style, ventrolateral view. I-L, Perugina denticula: I, aedeagus, lateral view; J, same, posterior view; K, connective, ventral view; L, style, ventrolateral view. M-P, Spinigina hirsuta: M, aedeagus, lateral view; N, same, posterior view; O, connective, ventral view; P, style, ventrolateral view. Q-T, Spinigina quadrispinosa: $\mathrm{Q}$, style, ventral view; R, connective, ventral view; $\mathrm{S}$, aedeagus, posterior view; $\mathrm{T}$, same, lateral view. 

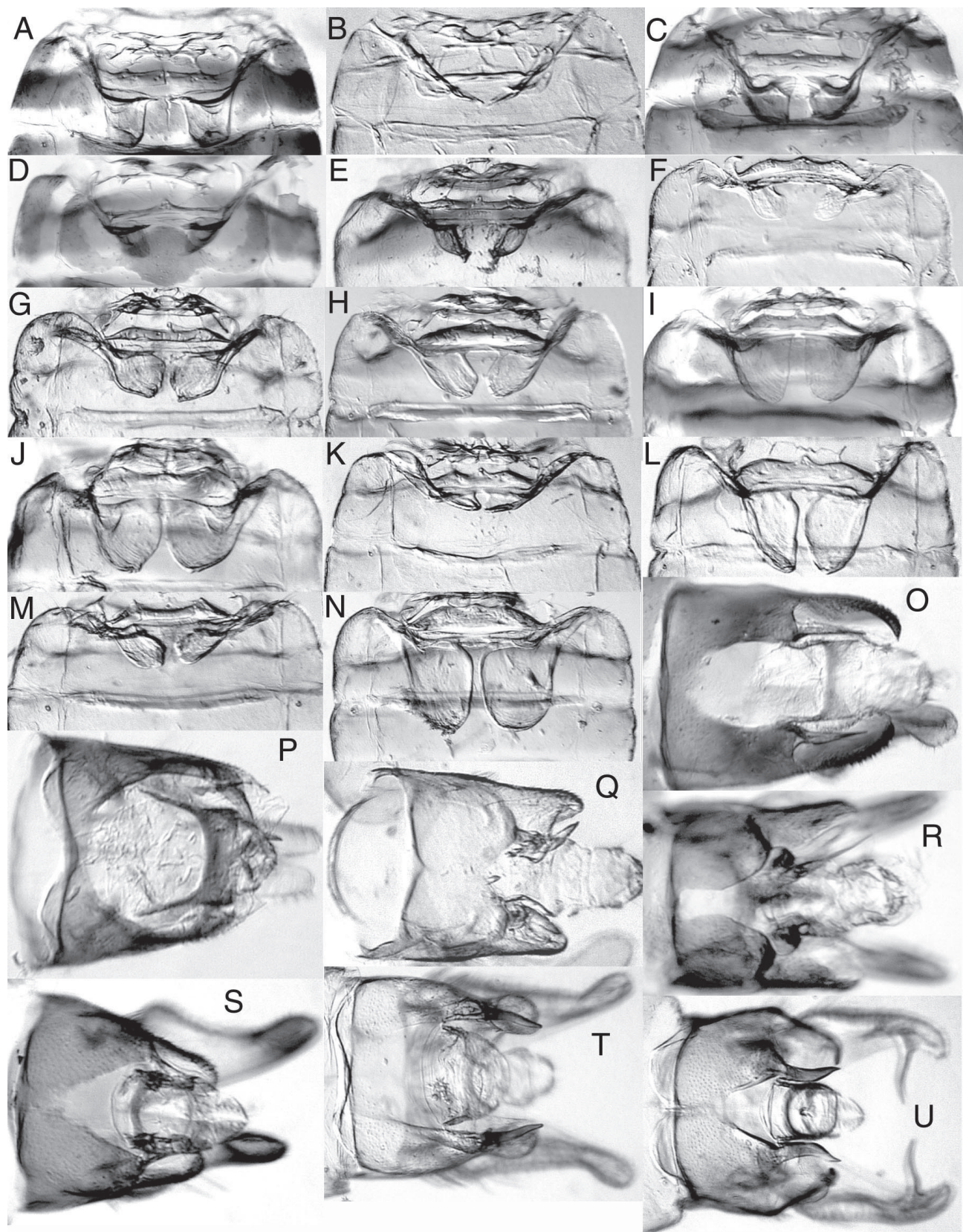

Figure 11. Male abdomen. A-N, base of abdomen ventral view: A, Aztegina punctinota; B, Mexigina oculata; C, Neozygina ceonothana; D, Nelionidia pueblensis; E, Amazygina chela; F, Amazygina compressa; G, Amazygina depressa; H, Amazygina decaspina; I, Hamagina pascoensis; J, Hamagina serrata; K, Hamagina spinigera; L, Napogina recta; M, Perugina denticula; $\mathrm{N}$, Spinigina hirsuta. O-U: genital capsule, dorsal view: O, Aztegina punctinota; $\mathrm{P}$, Neozygina ceonothana; Q, Nelionidia elliptica; R, Nelionidia moyai; S, Nelionidia pueblensis; T, Amazygina depressa; U, Hamagina spinigera. 


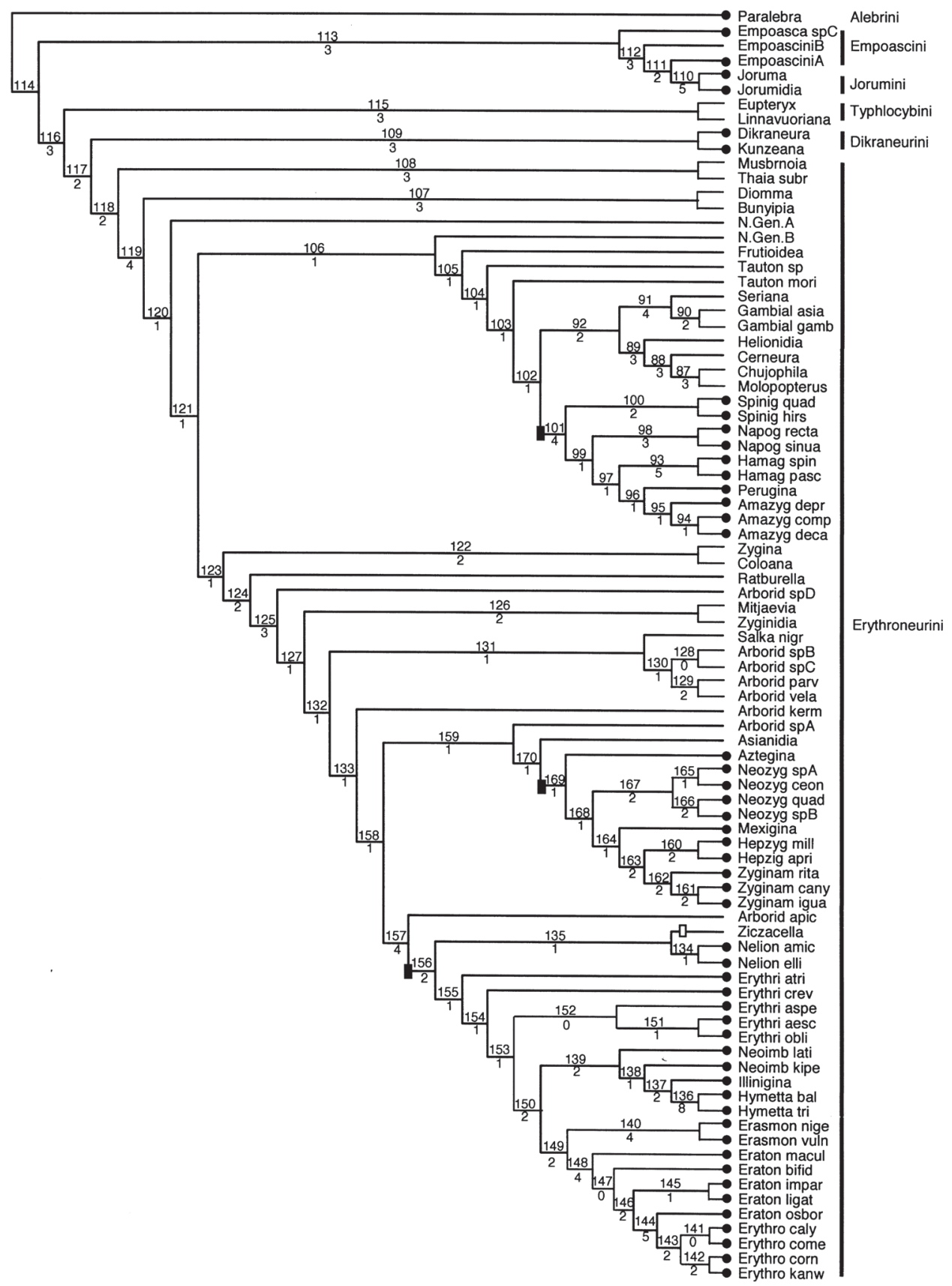

Figure 12. One of 24 equally parsimonious trees from phylogenetic analysis of 100 morphological characters. Apomorphies for numbered nodes and terminal taxa are given in Appendix D. Decay indices are given below the branches; nodes with decay index $=0$ collapsed on the strict consensus tree. Black circles at branch tips indicate New World taxa. Black rectangles on internal nodes indicate separate invasions of Erythroneurini into the New World, based on accelerated transformation optimization. The clear rectangle subtending Ziczacella indicates a "reversal" from New to Old World. This scenario is equally parsimonious to one in which separate invasions of the New World by the ancestor of Nelionidia and of the clade comprising Erythridula atrimucronata occurred. 
Illinois Natural History Survey

I-Building

1816 South Oak Street

Champaign, Illinois 61820

217-333-6880

A Division of the Illinois Department of Natural Resources

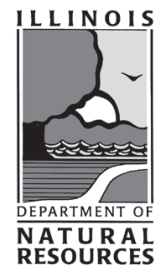

